This work is licensed under a Creative Commons Attribution License (CC BY 4.0).

Research article

urn:lsid:zoobank.org:pub:F8C5C34F-CCBB-420B-9319-1DA224A5D783

\title{
Revision of the Merodon bombiformis group (Diptera: Syrphidae) - rare and endemic African hoverflies
}

\author{
Ante VUJIĆ ${ }^{1}$, Snežana RADENKOVIĆ ${ }^{2}$, Ljiljana ŠAŠIĆ ZORIĆ ${ }^{3}$, Laura LIKOV ${ }^{4}$, \\ Tamara TOT ${ }^{5}$, Sanja VESELIĆ ${ }^{*, 6} \&$ Mihajla DJAN ${ }^{7}$ \\ 1,2,4,5,6,7 University of Novi Sad, Faculty of Sciences, Department of Biology and Ecology, \\ Trg Dositeja Obradovića 2, 21000 Novi Sad, Serbia. \\ ${ }^{3}$ University of Novi Sad, BioSense Institute, Dr Zorana Đinđića 1, 21000 Novi Sad, Serbia.
}

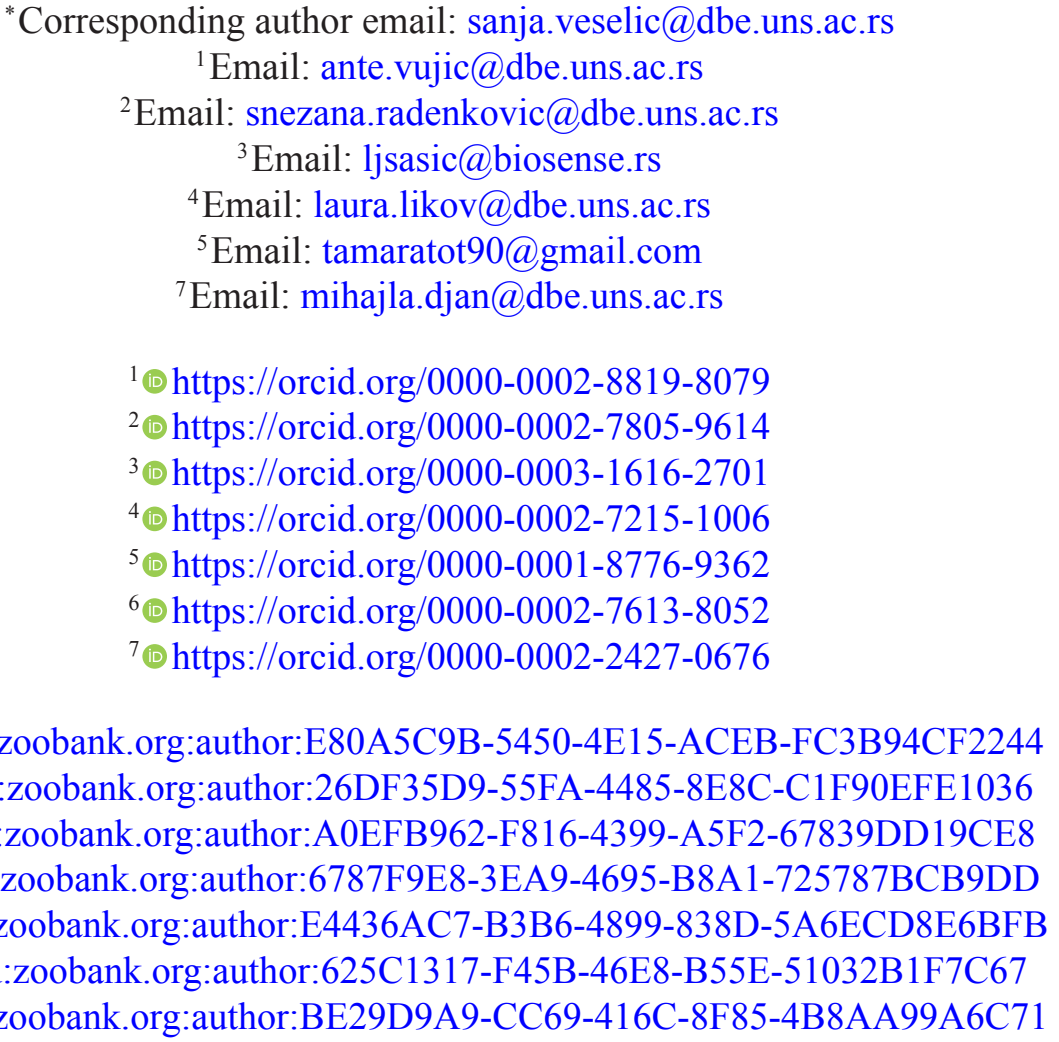

\begin{abstract}
In the present work, the Afrotropical species of the bombiformis species group, part of the aureus lineage, are revised. Six species are recognized, based on a combination of morphological and genetic features. Three of these species are new to science: Merodon lotus Vujić \& Radenković sp. nov., M. vittatus Vujić \& Likov sp. nov., and M. zebra Vujić \& Radenković sp. nov. Redescriptions are provided for the other three species: M. bombiformis Hull, 1944, M. multifasciatus Curran, 1939, and M. nasicus Bezzi, 1915. The female of M. bombiformis is described. The name Merodon edentulus Macquart, 1855 is considered here as a nomen dubium. One new synonymy is proposed: M. apimima Hull, 1944 syn. nov. (junior synonym of M. multifasciatus). The distribution of the bombiformis species
\end{abstract}


VUJIĆ A. et al., Revision of the Merodon bombiformis species group

group is discussed. The larval host plant of M. multifasciatus is identified as Gladiolus. A key to the identification of both males and females of the bombiformis group is provided.

Keywords. Afrotropical region, Merodon aureus lineage, molecular data, new species, syrphids, hoverfly, flower fly.

Vujić A., Radenković S., Šašić Zorić Lj., Likov L., Tot T., Veselić S. \& Djan M. 2021. Revision of the Merodon bombiformis group (Diptera: Syrphidae) - rare and endemic African hoverflies. European Journal of Taxonomy 755: 88-135. https://doi.org/10.5852/ejt.2021.755.1401

\section{Introduction}

The phytophagous hoverfly genus Merodon Meigen, 1803 (Diptera: Syrphidae: Eristalinae: Merodontini) is distributed across the Palaearctic and Afrotropical regions and comprises more than 160 species (Ståls et al. 2009; Vujić et al. 2012). Merodon equestris (Fabricius, 1794) as an introduced anthropogenic species from Europe is also widely distributed across the Nearctic (Speight 2020). The highest diversity of genus Merodon is recorded around the Mediterranean basin, a phenomenon which has been associated with the great variety of geophytes in this region, as those are the host plants of larvae of Merodon (Andric et al. 2014; Ricarte et al. 2017). Although the immature stages of only eight species of Merodon have been described and the larval host plants are postulated only for eight additional species, based on still incomplete evidence, the connection of larvae of Merodon with underground storage organs of the family Hyacinthaceae Batsch ex Borkh. is well recognized (Ricarte et al. 2008, 2017; Andrić et al. 2014; Preradović et al. 2018). It might be expected that the congruence between geophyte diversity and species-richness of Merodon, highlighted by Ricarte et al. (2017) for the European Mediterranean zone, would be paralleled in South Africa, since it is there that the geophyte diversity is at its maximum (Pfosser \& Speta 2004). But, surprisingly, nowhere within the Afrotropical Region is there a high diversity of species of Merodon.

Only 15 species consigned to the genus Merodon have been reported from the Afrotropical Region: Merodon apimima Hull, 1944, M. bombiformis Hull, 1944, M. capensis Hurkmans, 2018, M. capi Vujić \& Radenković, 2020, M. commutabilis Radenković \& Vujić, 2018, M. cuthbertsoni Curran, 1939, M. drakonis Vujić \& Radenković, 2018, M. edentulus Macquart 1855, M. flavocerus Hurkmans, 2018, M. melanocerus Bezzi, 1915, M. multifasciatus Curran, 1939, M. nasicus Bezzi,1915, M. planifacies Bezzi, 1915, M. roni Radenković \& Vujić, 2020 and M. stevensoni Curran, 1939 (Smith \& Vockeroth 1980; Radenković et al. 2018; Djan et al. 2020; Evenhuis \& Pape 2020). The low species diversity of Merodon in South Africa is the opposite of what might be anticipated (Radenković et al. 2018).

Bringing together previously published data (Šašić et al. 2016; Radenković et al. 2018), Vujić et al. (2019a), recognised five monophyletic lineages within the genus Merodon: albifrons, aureus, avidusnigritarsis, desuturinus, and natans. In the Afrotropical region, representatives of only two of these lineages are present, namely aureus and desuturinus.

The desuturinus lineage represents an important link between the Palaearctic and Afrotropical faunas. Palaearctic representatives of the group were revised by Vujić et al. (2018), resulting in the delimitation of four species: Merodon cabanerensis Marcos-García, Vujić \& Mengual, 2007 from Spain and Morocco, M desuturinus Vujić, Šimić \& Radenković, 1995, a Balkan endemic from high mountains, M. neolydicus Vujić, 2018 from the Eastern Mediterranean and Iran, and M. murorum (Fabricius, 1794), from North-West Africa. The Afrotropical component of the desuturinus lineage comprises 10 taxa: M. cuthbertsoni, plus the M. melanocerus subgroup with five species (M. capensis, M. commutabilis, M. drakonis, M. flavocerus and M. melanocerus) (Radenković et al. 2018) and the 
M. planifacies subgroup comprising two previously known (M. planifacies and M. stevensoni) and two recently recognized cryptic taxa: M. capi and M. roni (Djan et al. 2020).

The rest of the Afrotropical species of the genus Merodon belong to the aureus lineage. Šašić et al. (2016) defined species groups within this lineage, such as the M. aureus, M. bombiformis, M. funestus, M. nanus and M. spinitarsis species groups. Revision of all available material from museums and detailed analyses of newly collected specimens from our own expeditions to RSA resulted in the delimitation of the six species considered here, all belonging to the M. bombiformis group.

The aims of the present study were:

1) To review material belonging to the bombiformis group held in the collections of several entomological institutions and private individuals,

2) To define and describe the taxa within the bombiformis species group, including mapped geographic distributions and new species,

3) To examine the genetic diversity exhibited among the members of this species group and infer the phylogenetic relationship, using one mitochondrial gene (cytochrome c oxidase subunit I (COI)) and two nuclear genes (28S rRNA and 18S rRNA),

4) To present an illustrated key and a discussion on the different taxonomic characters used,

5) To discuss the larval host plant data available for the bombiformis species group.

\section{Material and methods}

\section{Morphological study}

The present study is based on the examination of all available material of the Merodon bombiformis species group deposited in the museums and universities listed below. The following abbreviations of museums and entomological collections are used in the text to indicate where the material studied is deposited:

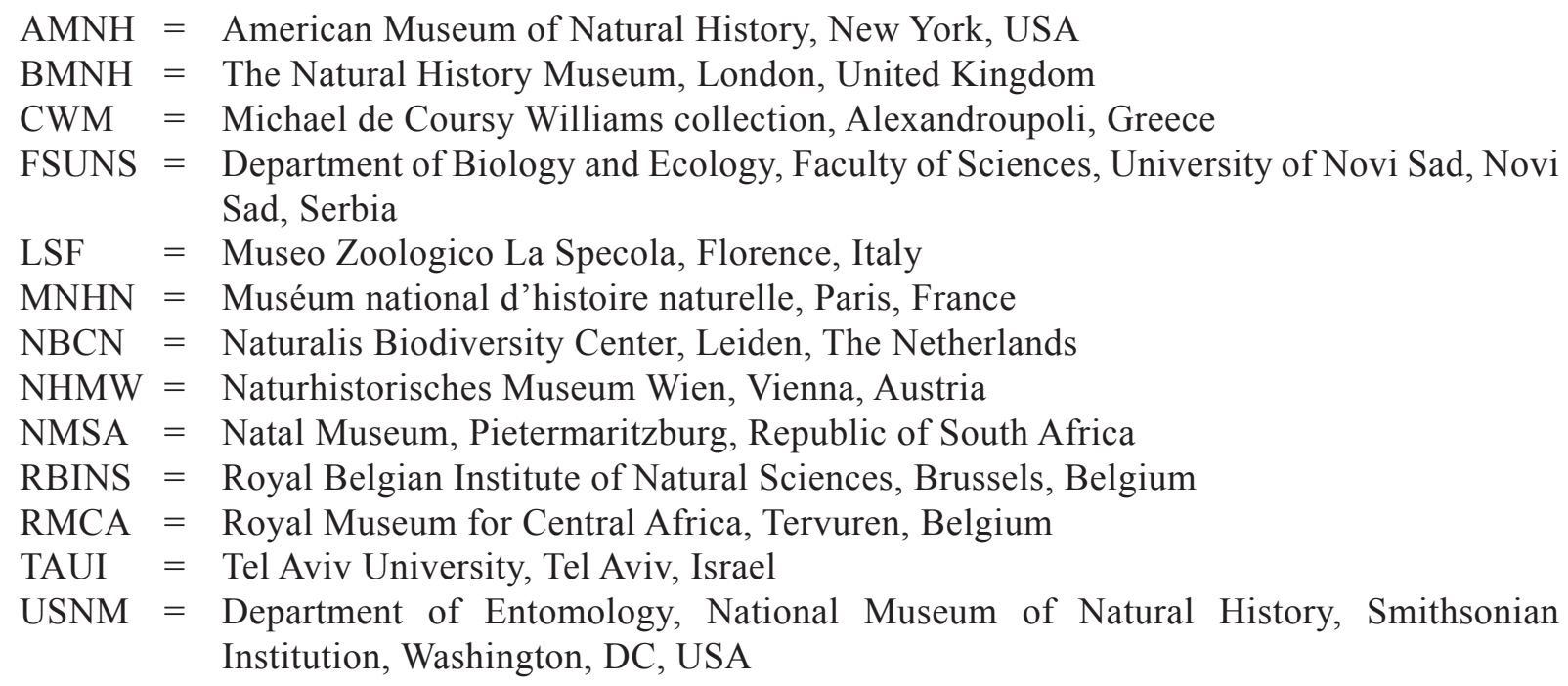

Numbers given alongside the abbreviation FSUNS in the examined material refer to unique identifiers of the specimens, stored in the database of the Faculty of Sciences, University of Novi Sad. The distribution map (Fig. 1) was generated with the mapping software DIVA-GIS (Hijmans et al. 2012). Relationships of species to different vegetation types were based on the map of natural vegetation of Africa (Sayre et al. 2013). 
The terminology used in the descriptions and drawings follows Thompson (1999) for non-genitalic features except for the terms "proepimeron" and "pleuron", which follows McAlpine (1981), and those relating to male genitalia follow Marcos-García et al. (2007), while the term "fossette" follows Doczkal \& Pape (2009).

For the study of male genitalia, dry specimens were relaxed in a humidity chamber. The genitalia were extracted with a hook-tipped entomological pin before being cleared by boiling in warm $10 \%$ potassium hydroxide $(\mathrm{KOH})$ for 3-5 min. This was followed by brief immersion in acetic acid to neutralize the $\mathrm{KOH}$, and then by immersion in ethanol to remove the acid. The prepared genitalia were stored in microvials containing glycerol pinned to the source specimen.

Digital photographs were taken with a Leica DFC 320 digital camera attached to a Leica MZ16 stereo microscope, while for drawings a Leica MZ16 binocular microscope was used with an FSA 25 PE drawing tube. The photographs were stacked using CombineZ software (Hadley 2006).

Body length was measured in lateral view in millimetres $(\mathrm{mm})$ using an eyepiece micrometer from the lunula to the end of the abdomen. Wing length was measured from the tegula to the apex of the wing.
Abbreviations
ams $=$ anterior margin of surstyle
$\mathrm{c} \quad=$ cercus
ea $=$ ejaculatory apodeme
$\mathrm{p} \quad=$ aedeagus
$\mathrm{pl}=$ posterior lobe of surstyle
$\mathrm{s} \quad=$ lateral sclerite of aedeagus

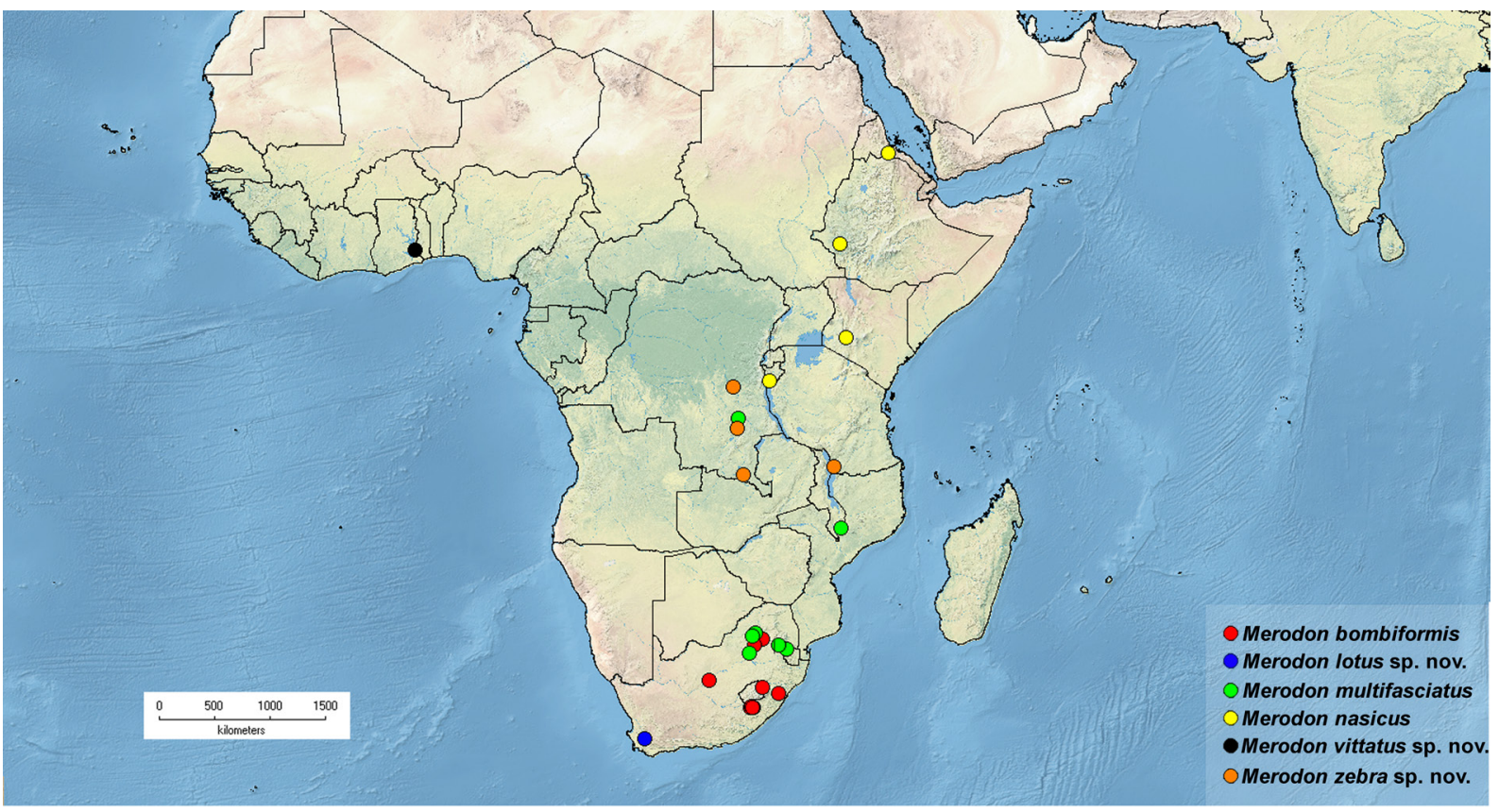

Fig. 1. Distribution map of the Merodon bombiformis species group. 


\section{Molecular analysis}

The genomic DNA of six hoverfly specimens, belonging to three species from the Merodon bombiformis species group (M. bombiformis, M. multifasciatus and M. lotus Vujić \& Radenković sp. nov.) was extracted from mid and hind legs using the sodium dodecyl sulfate (SDS) extraction protocol described by Chen et al. (2010). Fresh material required for high-quality DNA extraction was only available for these species. Species of the M. bombiformis group for which we were unable to obtain fresh material were not included. Genomic DNA vouchers are deposited at the Faculty of Sciences, Department of Biology and Ecology, University of Novi Sad (FSUNS).

We amplified one mitochondrial (COI gene) and two nuclear genes (28S rRNA and 18S rRNA genes). The Folmer fragment (DNA barcode) on 5'COI gene was amplified using LCO1490 and HCO2198 primer pair (Folmer et al. 1994), the 28S rRNA gene using F2 and 3DR primer pair (Belshaw et al. 2001), while for amplification of $18 \mathrm{~S}$ rRNA we used $2 \mathrm{~F}$ and $\mathrm{b} 2.9$ primer pair designed by The Crandall Lab (for primer sequences see Vujić et al. 2019b). Polymerase chain reactions (PCR) were carried out in $25 \mu \mathrm{l}$ reaction volumes. The reaction mixture contained Dream Taq buffer (ThermoScientific, Vilnius, Lithuania), $0.1 \mathrm{mM}$ of each nucleotide, 5 pmol of each primer, 1.25U Dream Taq polymerase (ThermoScientific, Vilnius, Lithuania), $\sim 50-100 \mathrm{ng}$ of template DNA and ultrapure water. PCR amplifications were performed using the following conditions: $95^{\circ} \mathrm{C}$ for 2 min; 29 cycles of $94^{\circ} \mathrm{C}$ for $30 \mathrm{~s}$ each, $50^{\circ} \mathrm{C}$ for $30 \mathrm{~s} ; 72^{\circ} \mathrm{C}$ for $2 \mathrm{~min}$; with a final extension at $72^{\circ} \mathrm{C}$ for $8 \mathrm{~min}$. PCR products were purified using Exonuclease I and FastAP Thermosensitive Alkaline Phosphatase (Thermo Scientific, Vilnius, Lithuania) according to the manufacturer's instructions. The PCR fragments were commercially sequenced in the forward direction by Macrogen Europe (Amsterdam, Netherlands).

\section{Sequence processing and phylogenetic analysis}

The COI, 28S rRNA and 18S rRNA gene sequences were edited for base-call errors using BioEdit ver. 7.0.9.0. (Hall 1999). Additional sequences of several species representing the main Merodon lineages following Radenković et al. (2018) were downloaded from GenBank and joined to the sequence matrices of all tree genes. The COI and $18 \mathrm{~S}$ rRNA gene sequences were aligned manually, while 28S rRNA gene sequences were aligned using MAFFT ver. 7 (Katoh \& Standley 2013), available from the European Bioinformatics Institute (EMBL-EBI) web services (McWilliam et al. 2013). The aligned COI, $28 \mathrm{~S}$ rRNA and $18 \mathrm{~S}$ rRNA gene sequences were concatenated and combined into a single sequence matrix. The ML tree was constructed using RAxML ver. 8.2.8 (Stamatakis 2014) using the CIPRES Science Gateway web portal (Miller et al. 2010) under the general time-reversible (GTR) evolutionary model with a gamma distribution (GTRGAMMA) (Rodríguez et al. 1990) following instructions in the manual. The applied command line was as follows: raxmlHPC-HYBRID -T 4 -n result -s infile.txt -m GTRGAMMA -p 12345 -q part.txt -f a -N 1000 -x 12345 -o E._pulchel. Nodal supports were estimated using rapid bootstrapping with 1000 replicates. The tree is rooted with Eumerus pulchellus Loew, 1848 as outgroup. The uncorrected pairwise (p) distance matrix for 5' COI gene sequences among specimens of the M. bombiformis species group was estimated using MEGA ver. 7 software (Kumar et al. 2016). GenBank accession numbers of all sequences are provided in Supp. file 1. 


\title{
Results
}

\section{Taxonomic revision}

\author{
Phylum Arthropoda Latreille, 1829 \\ Class Insecta Linnaeus, 1758 \\ Order Diptera Linnaeus, 1758 \\ Family Syrphidae Latreille, 1802 \\ Subfamily Eristalinae Newman, 1834 \\ Tribus Merodontini Edwards, 1915
}

Genus Merodon Meigen, 1803

\section{Diagnosis of the Merodon aureus lineage}

Mid coxa with long pile posteriorly (Fig. 2A); anterior anepisternum below postpronotum with many long pile (Fig. 2B); species with stocky abdomen (Fig. 2C); lateral sclerites of the aedeagus very small or absent (Fig. 3A: marked with arrow).

\section{Diagnosis of the Merodon bombiformis group}

Pedicel elongated, approximately as long as basoflagellomere (Fig. 4); abdomen broad (Fig. 5); metafemur with less serrated apicoventral triangular lamina, usually only the apical dens is distinct (Fig. 6); metatrochanter of males smooth, without calcar (Fig. 6); male genitalia with posterior surstyle lobe usually bent (as on Fig. 7A, D: pl), and hypandrium narrowed medially (as on Fig. 7C: marked with arrow); distribution: Afrotropical Region (Fig. 1).

The Merodon bombiformis group and M. funestus (Fabricius, 1794) differ from other species and groups among the aureus lineage by an elongated pedicel, approximately as long or even longer than basoflagellomere (Fig. 4) and small lateral sclerite of the aedeagus (as on Fig. 3B, D: s) (absent in other species and groups of the aureus lineage, on Fig. 3A: marked with arrow). Morphologically, the $M$. bombiformis group can be distinguished from $M$. funestus by the absence of a calcar on metatrochanter in males (present in M. funestus, on Fig. 8A), less dentate apicoventral triangular lamina on the metafemur, but usually with a distinct apical dens (Fig. 8B) (clearly dentate in M. funestus, on Fig. 8A) and by the shape of the posterior lobe of the surstylus: tip rounded in bombiformis group (as on Fig. 13A, D: pl), but tapering in M. funestus (Fig. 19A: pl).

\section{Merodon bombiformis Hull, 1944}

Figs 1, 3C, 4A, 5A, 6A, 9A, 10A, 11A, 12A, 13A-C, 14A, 15A, 16A, 21A-D, 24

Merodon bombiformis Hull, 1944: 42-43 (type locality: Waterval, Republic of South Africa).

\section{Diagnosis}

Large, golden species (10-14 mm) with reddish-yellow/golden face and frons (Figs 9A, 11A), lateral sides of scutum (Fig. 10A) and most of terga (Fig. 5A); oral margin reduced (Fig. 9A); antennal segments very short (Fig. 4A), frons inflated (Fig. 9A).

\section{Material examined}

\section{Holotype}

REPUBLIC OF SOUTH AFRICA • 1 ơ; Waterval; $25.183^{\circ}$ S, 29.012 ${ }^{\circ}$ E; 27 Dec. 1898; Distant Collection 1911:383; BMNH. 


\section{Additional material}

REPUBLIC OF SOUTH AFRICA • 1 đ’; Distant Bush; $28.586^{\circ} \mathrm{S}, 24.658^{\circ} \mathrm{E} ; 1182 \mathrm{~m}$ a.s.1.; 1911;

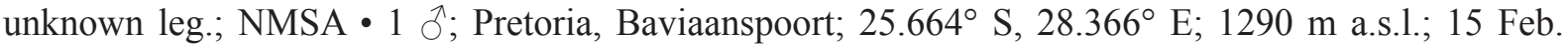
1920; H.K. Munro leg.; FSUNS ID 04277; NMSA • 1 क; Natal, Hilton Road; $29.597^{\circ}$ S, $30.336^{\circ}$ E; 833 m a.s.1.; 22 Dec. 1953; P. Graham leg.; FSUNS ID 04280; NMSA • 1 ㅇ; Pietermaritzburg; $29.597^{\circ}$ S, $30.336^{\circ}$ E; 833 m a.s.1.; 5 Nov. 1955; Oosthuizen leg.; reared from Gladiolus bulbs; FSUNS ID 04282;

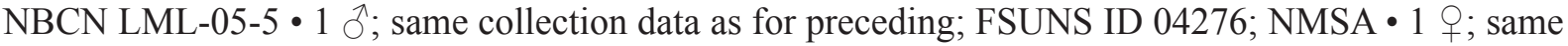
collection data as for preceding; 19 Nov. 1955; FSUNS ID 04279; NMSA • 1 ○; reared from Gladiolus bulbs in Wisley Horticultural Gardens (United Kingdom), which were brought from RSA; Jun. 1956;

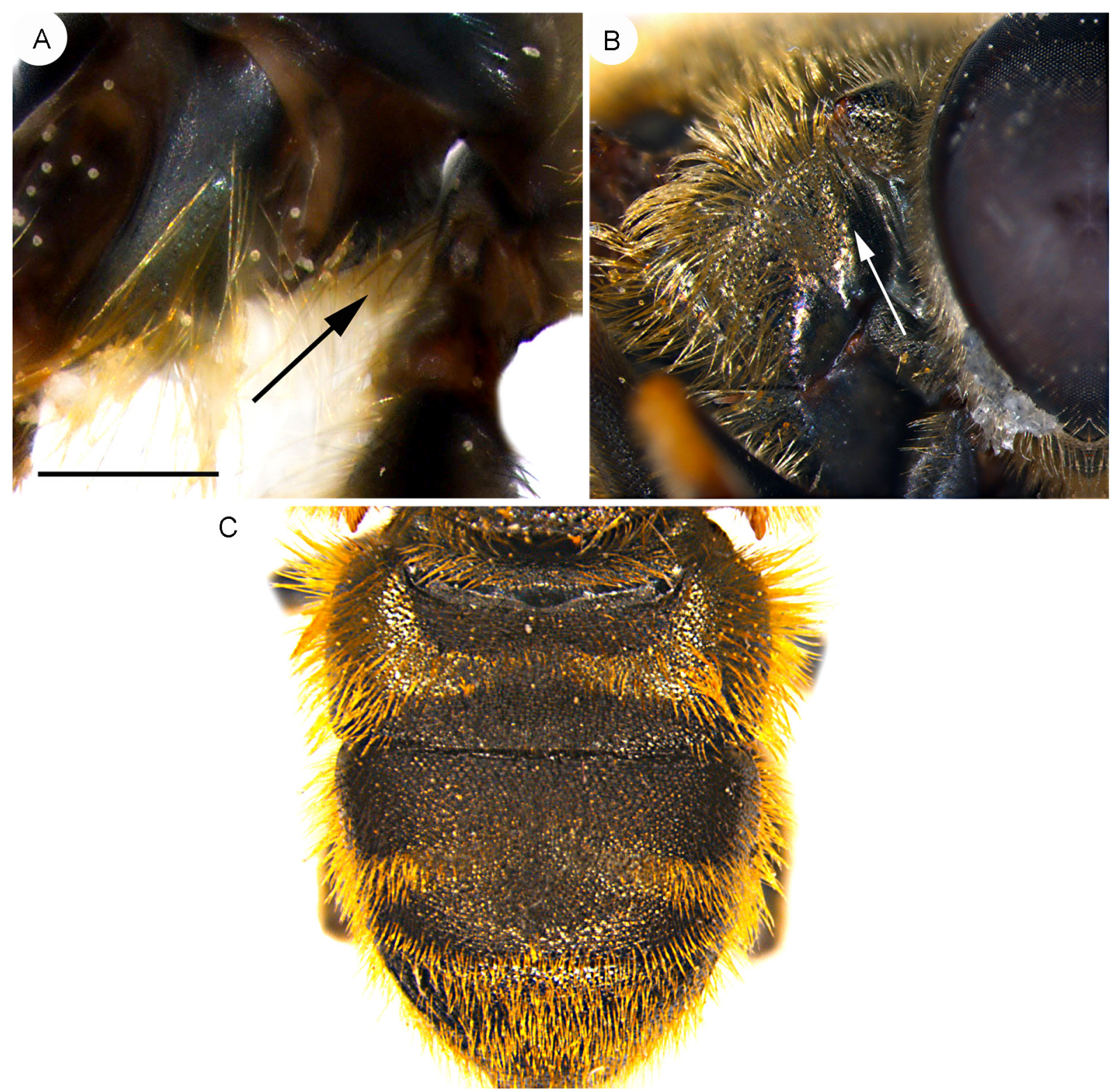

Fig. 2. A-C. Merodon funestus (Fabricius, 1794), ठ․ A. Mid coxa (FSUNS; FSUNS ID F98), lateral view, posterior pile marked with arrow. B. Anterior anepisternum (CWM; FSUNS ID 10347), pile below postpronotum marked with arrow, anterolateral view. C. Abdomen, dorsal view (FSUNS; FSUNS ID F99). Scale bar $=2 \mathrm{~mm}$. 
V.W. Fowler leg.; BMNH 1 1 ; Transkei, Pitseng Pass, banks from Luzzi River; 30.749 S, 28.299 E; 1633 m a.s.1.; 11 Jan. 1979; J. Londt and B. Stuckenberg leg.; rocky hill and grassveld; FSUNS ID 04278; NMSA • 1 万ै; Eastern Cape, 5 km ENE of Rhodes; $30.783^{\circ} \mathrm{S}, 28.000^{\circ} \mathrm{E}$; $2066 \mathrm{~m}$ a.s.1.; 5 Feb. 1992; wet stream gully; unknown leg.; FSUNS ID 04275; NMSA • 1 क ; Eastern Cape, Naudes Nek, near top of pass; $30.759^{\circ} \mathrm{S}, 28.137^{\circ} \mathrm{E}$; $2500 \mathrm{~m}$ a.s.1.; 6 Feb. 2009; S.I. Morita leg.; FSUNS ID 05724; USNM SMPC_SIM_1844 • 1 万; ; Drakensberg Mountain, Maclear-Naudes Nek; $30.759^{\circ}$ S, $28.203^{\circ}$ E; $1800 \mathrm{~m}$ a.s.1.; 9 Feb. 2016; S. Radenković and N. Veličković leg.; FSUNS ID ZA2_079; FSUNS.

LESOTHO • 1 क; Colony of Basutoland, Orange Valley; $29.15^{\circ} \mathrm{S}, 29.03^{\circ} \mathrm{E} ; 1500 \mathrm{~m}$ a.s.l.; R. Ellenberger leg.; FSUNS ID 03317; MNHN PM-05-46.

\section{Redescription}

Original description is insufficient, without any illustrations and based on a single male specimen from South Africa (Hull 1944).

Length: large species, body 10-14 mm, wing 8-10 mm $(\mathrm{n}=8)$.

\section{Male}

HeAD (Figs 4A, 9A, 11A, 12A). Antenna (Fig. 4A) very short, reddish to dark brown; scape and pedicel covered with dense, yellow pilosity; pedicel elongated, approximately as long as basoflagellomere (relation scape: pedicel: basoflagellomere $=1.0: 1.8: 1.8$ ); basoflagellomere concave dorsally, with

A

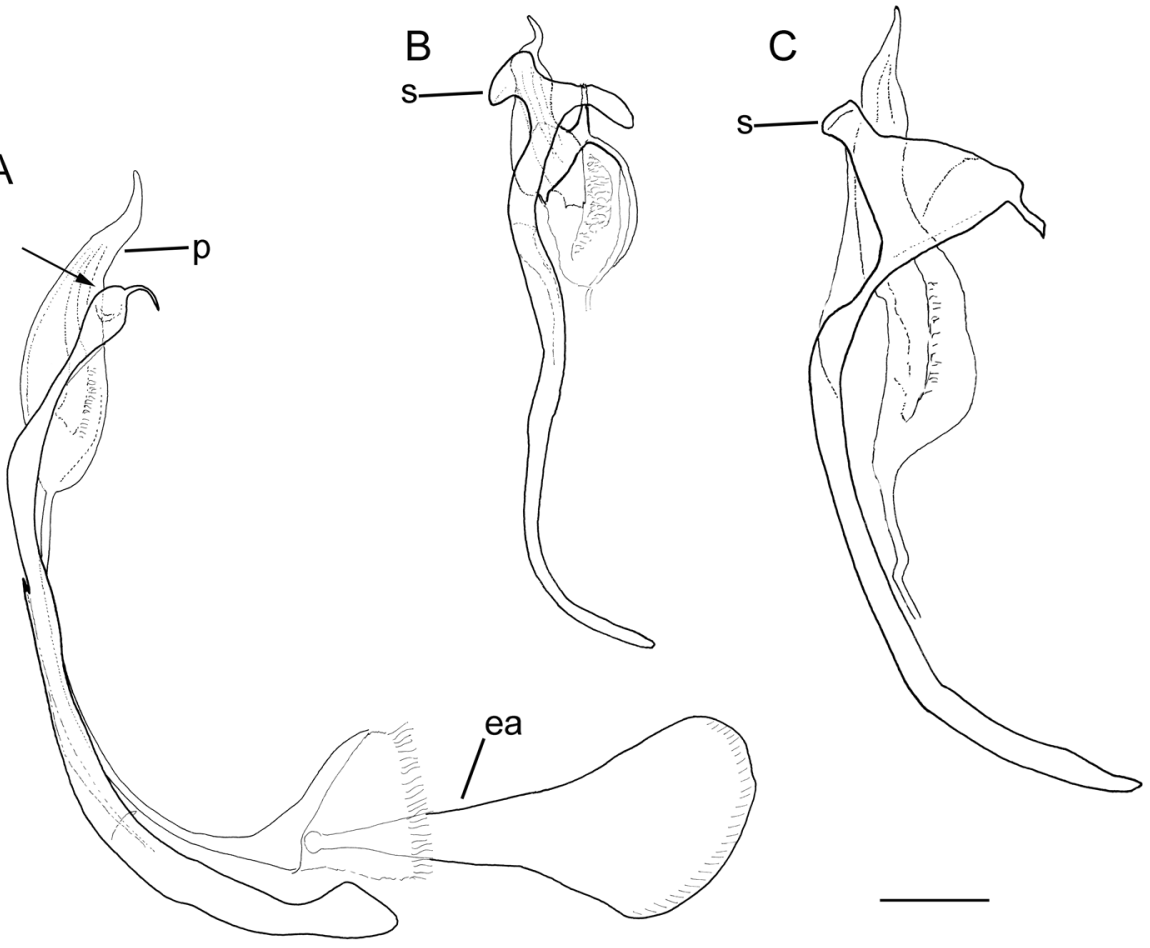

D

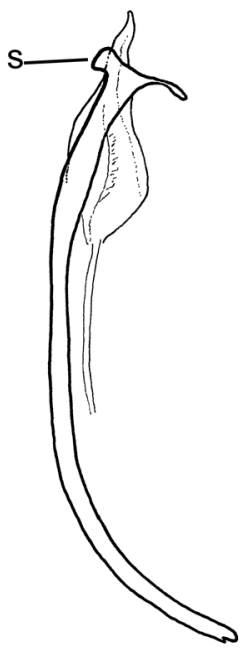

Fig. 3. Male genitalia, aedeagus, lateral view. A. Merodon aureus (Fabricius, 1805) (FSUNS; FSUNS ID 09662) the place of absence of lateral sclerite of the aedeagus marked with arrow. B. M. funestus (Fabricius, 1794) (FSUNS; FSUNS ID F99). C. M. bombiformis Hull, 1944 (FSUNS; FSUNS ID A2_079). D. M. zebra Vujić \& Radenković sp. nov., paratype (BMNH; FSUNS ID 03314). Abbreviations: $\mathrm{ea}=$ ejaculatory apodeme; $\mathrm{p}=$ aedeagus; $\mathrm{s}=$ lateral sclerite of aedeagus. Scale bar $=0.25 \mathrm{~mm}$. 


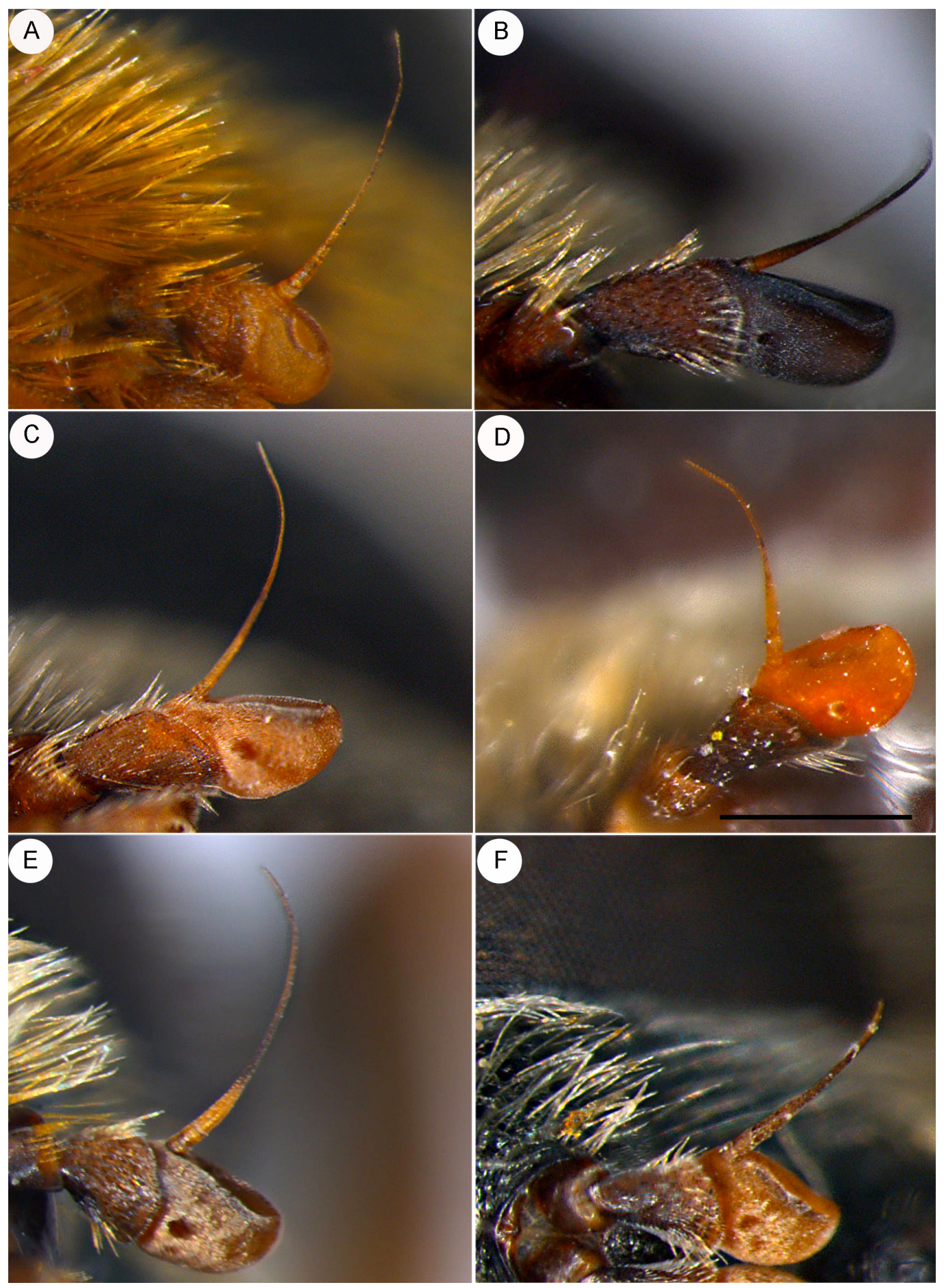

Fig. 4. Male antenna, lateral view. A. Merodon bombiformis Hull, 1944 (FSUNS; FSUNS ID ZA2_079). B. M. multifasciatus Curran, 1939 (USNM; FSUNS ID 04512). C. M. vittatus Vujić \& Likov sp. nov., holotype (NBCN; FSUNS ID 04085). D. M. zebra Vujić \& Radenković sp. nov. paratype (NBCN; FSUNS ID 05174). E. M. lotus Vujić \& Radenković sp. nov., paratype (FSUNS; FSUNS ID ZA6_066). F. M. nasicus Bezzi, 1915 (TAUI; FSUNS ID 04972). Scale bar $=2 \mathrm{~mm}$. 
rounded apex; arista light brown to dark brown, thickened basally, 5 times as long as basoflagellomere. Face reddish-yellow to dark brown, covered with yellow pollinosity and dense yellow pile, except on bare medial vitta that occupies $1 / 4$ width of face (Fig. 11A). Oral margin reduced (Fig. 9A). Frons from black to reddish and golden-yellow, inflated, covered with golden-yellow pollinosity and pile. Vertical triangle isosceles (Fig. 12A), usually reddish to black, predominantly covered with a long, yellow pile. Ocellar triangle equilateral. Eye pile dense, long as the scape, often pale yellow to gray. Eye contiguity about 10-15 ommatidia long. Occiput reddish, covered with yellow pile.

Thorax (Figs 6A, 10A). Mesonotum matte black, except lateral side of scutum including postpronotum and postalar callus, and posterior margin of scutellum reddish-yellow, covered with reddish pile; scutum with five pollinose longitudinal vittae (Fig. 10A). Pleuron covered with gray pollinosity and the following parts with whitish pile: anterior part of proepimeron, posterior part of anterior anepisternum, most of the posterior anepisternum except anterior end, antero-ventral and postero-dorsal part of katepisternum, anepimeron, and metasternum; in some specimens anterior anepisternum and katatergum reddish. Wing hyaline, with dense microtrichia and yellow to brown veins. Calypter pale yellow. Halter with yellow pedicel and capitulum. Legs usually dark brown to black (in some specimens partly reddish), except yellow basal third of tibiae. Metatrochanter without calcar. Metafemur moderately thickened, with less developed apicoventral triangular lamina, not dentate, only the apical dens is distinct, posteriorly oriented (Fig. 6A). Pile on legs predominantly yellow.

AвDOMEn (Fig. 5A). Predominantly reddish-yellow (Fig. 5A). Reddish parts of terga 2-4 covered with yellow pile and dense pollinosity, while dark parts with short adpressed black pile; sterna from yellow to dark brown, covered with white or yellow pile.

Genitalia (Figs 3C, 13A-C). Posterior lobe of surstyle broad, rounded apically (Fig. 13A: pl); anterior lobe of surstyle undeveloped, virtually absent (Fig. 13A); cercus elongated (Fig. 13A: c). Hypandrium with theca slightly medially narrowed (Fig. 13C: marked with arrow). Lateral sclerite of aedeagus small, quadrilateral (Fig. 3C: s).

Female (Figs 14A, 15A, 16A)

Similar to the male except for normal sexual dimorphism: frons reddish-yellow, with broad goldish pollinose vittae along eye margins; frons completely covered with golden pile (Fig. 15A); metafemur with very small apicoventral triangular lamina; apical dens more or less distinct, slightly posteriorly oriented (Fig. 16A).

\section{Period of flight and distribution (Fig. 1)}

The species is distributed in the Republic of South Africa and Lesotho. On the basis of our data, the flight period was found to be from November to February. Merodon bombiformis was collected in different vegetation types, brush-grass savanna, temperate and mountain grasslands and montane foresttundra. On Drakensberg Mountain, at the locality between Maclear and Naudes Nek, it was found near the stream, within bushes of Leucosidea sericea Eckl. \& Zeyh. (Rosaceae) and Gladiolus sp. on rocky soil (Fig. 24). Leucosidea sericea grows in Afromontane regions of southern Africa, and is the sole species of this genus (South African National Biodiversity Institute 2004).

\section{Larval biology}

Stuckenberg (1956) described the puparia of $M$. bombiformis, reared from larvae found feeding on commercially grown Gladiolus sp. (Iridaceae) corms. Specimens from Pietermaritzburg (5 Nov. 1955, 19 Nov. 1955) were reared from bulbs of Gladiolus L. too. 

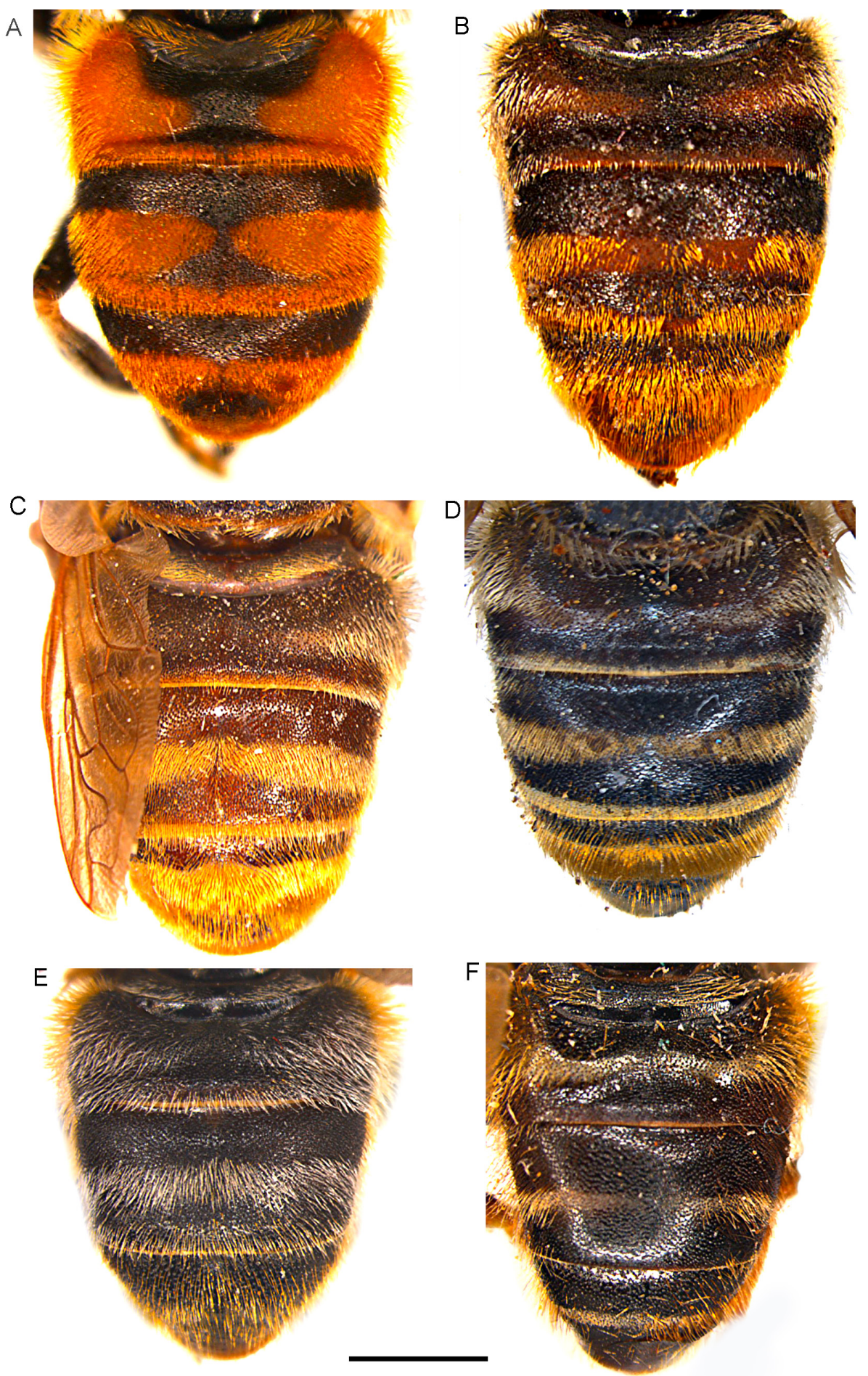

Fig. 5. Male abdomen, dorsal view. A. Merodon bombiformis Hull, 1944 (FSUNS; FSUNS ZA2_079). B. M. multifasciatus Curran, 1939 (USNM 2052372; FSUNS ID 04512). C. M. vittatus Vujić \& Likov sp. nov. holotype (NBCN; FSUNS ID 04085). D. M. zebra Vujić \& Radenković sp. nov. holotype (RMCA; FSUNS ID 25089). E. M. lotus Vujić \& Radenković sp. nov., paratype (FSUNS; FSUNS ID ZA6_066). F. M. nasicus Bezzi, 1915 (RBINS; FSUNS ID 21976). Scale bar $=2 \mathrm{~mm}$. 
Merodon lotus Vujić \& Radenković sp. nov. urn:1sid:zoobank.org:act:4FA6E871-8E78-418E-89EA-B4A334742811

Figs 1, 4E, 5E, 6E, 9E, 10E, 11E, 12E, 14D, 15D, 16D, 17, 18A, 22A-C, 26

\section{Diagnosis}

Black, medium sized species (8-9 mm), with shiny face, posterior half of scutum and terga without pollinosity (except a pair of indistinct pollinose fasciae on tergum 4 in female) (Fig. 14D); male genitalia with S-forming posterior lobe of surstyle, gradually narrowing toward tip (Fig. 17A, D: pl), anterior margin of surstyle rounded in lateral view (Fig. 17A, D: ams). Similar to Merodon nasicus from which differs in less prominent oral margin (Fig. 11E), and terga without clear pollinosity (Figs 5E, 14D), while in M. nasicus oral margin distinctly protruded (Fig. 11F) and terga 2-4 with distinct pollinose fasciae (Figs 5F, 14E, 20D).

\section{Etymology}

The specific epithet 'lotus' is a Latin adjective meaning 'elegant, fine', also 'washed/clean', implying clean, non pollinose terga, as the main diagnostic character of this species.

\section{Material examined}

\section{Holotype}

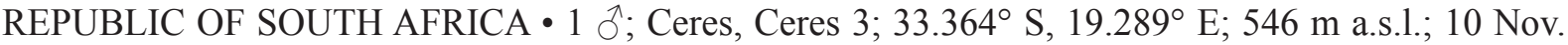
2018; A. Vujić and S. Radenković S. leg.; FSUNS ID ZA6_039; NMSA.

\section{Paratypes}

REPUBLIC OF SOUTH AFRICA 1 ô; Ceres, Ceres 2; $33.365^{\circ} \mathrm{S}, 19.289^{\circ} \mathrm{E} ; 547 \mathrm{~m}$ a.s.1.; 9 Nov.

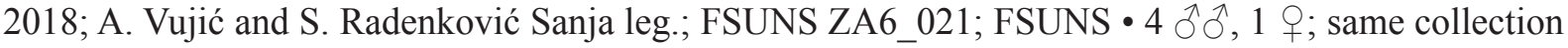
data as for holotype; FSUNS ZA6_048, ZA6_049, ZA6_052, ZA6_060, ZA6_066; FSUNS • 1 q; same collection data as for holotype; FSUNS ID ZA6_067; NMSA.

\section{Additional material}

REPUBLIC OF SOUTH AFRICA • 1 ô; Cape Province, Ceres; 1500 ft; Dec. 1920; R.E. Turner leg.; Merodon appendiculatus Hull unpublished name; BMNH.

\section{Description}

Length: medium sized species, body $8-9 \mathrm{~mm}$, wing $5-6 \mathrm{~mm}(\mathrm{n}=8)$.

\section{Male}

HAD (Figs 4E, 9E, 11E, 12E). Antenna (Fig. 4E) brown; pedicel slightly shorter than basoflagellomere (relation scape:pedicel: basoflagellomere $=1.0: 2.1: 2.5$ ); basoflagellomere concave dorsally, with acute apex; arista thickened basally, yellowish at basal third, the rest blackish, 2.2 times as long as basoflagellomere. Face shiny black, covered with whitish or yellowish pile, except on bare medial vitta that occupies half width of face. Oral margin slightly protruded (Fig. 9E). Frons shiny black, inflated, covered with whitish-gray pile and gray pollinosity along eye margins. Vertical triangle isosceles (Fig. 12E), dark, predominantly covered with long, yellowish pile, except some black ones on ocellar triangle in some specimens. Ocellar triangle usually equilateral. Eye pile gray, except upper fourth with black pile, slightly longer than scape. Eye contiguity about 14-16 ommatidia long. Occiput blackish, mostly pollinose, except behind vertical triangle, covered with yellow pile.

THORAX (Figs 6E, 10E). Mesonotum black, covered with reddish-yellow pile; scutum without pollinose longitudinal vittae, but postpronotum and anterior part of scutum pollinose (Fig. 10E). Pleuron black, covered with sparse gray pollinosity and the following parts with whitish to yellow pile: anterior part 

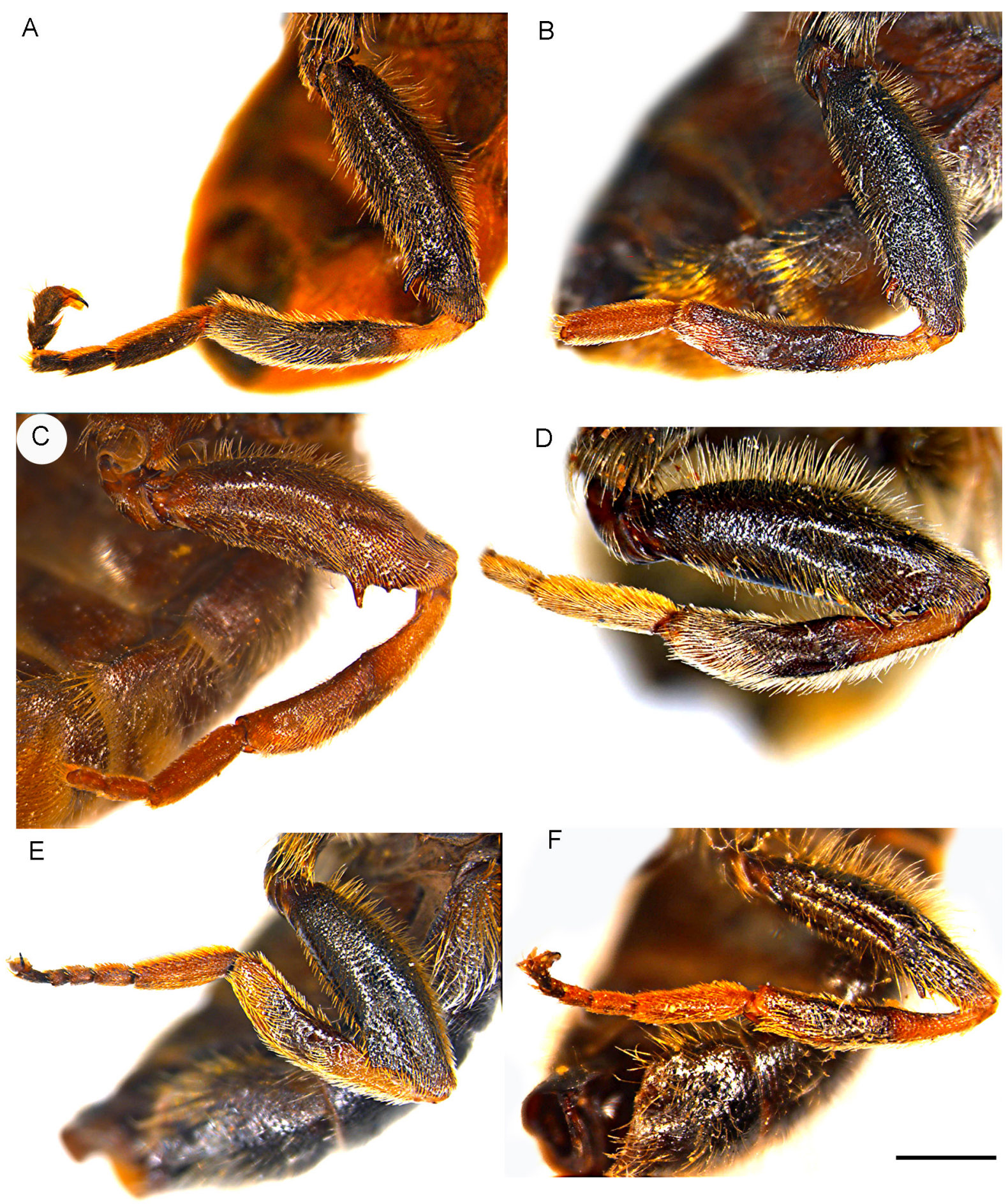

Fig. 6. Male metaleg, lateral view. A. Merodon bombiformis Hull, 1944 (FSUNS; FSUNS ZA2 079). B. M. multifasciatus Curran, 1939 (USNM 2052372; FSUNS ID 04512). C. M. vittatus Vujić \& Likov sp. nov., holotype (NBCN; FSUNS ID 04085). D. M. zebra Vujić \& Radenković sp. nov., paratype (NBCN; FSUNS ID 05174). E. M. lotus Vujić \& Radenković sp. nov., paratype (FSUNS; FSUNS ID ZA6_066). F. M. nasicus Bezzi, 1915 (TAUI; FSUNS ID 04972). Scale bar $=2 \mathrm{~mm}$. 
of proepimeron, posterior part of anterior anepisternum, most of the posterior anepisternum except anterior end, antero-ventral and postero-dorsal part of katepisternum, anepimeron, metasternum. Wing brownish, hyaline, with dense microtrichia and dark brown veins. Calypter yellowish. Halter with yellowish pedicel and capitulum. All three femora dark brown to black; tibiae mostly reddish, medially black to dark brown; tarsi reddish, except blackish apical segment (Fig. 6E). Metatrochanter without calcar. Metafemur moderately thickened, with less developed apicoventral triangular lamina, more or less denatate, the apical dens more or less distinct (Fig. 6E). Pile on legs predominantly yellowish.

A

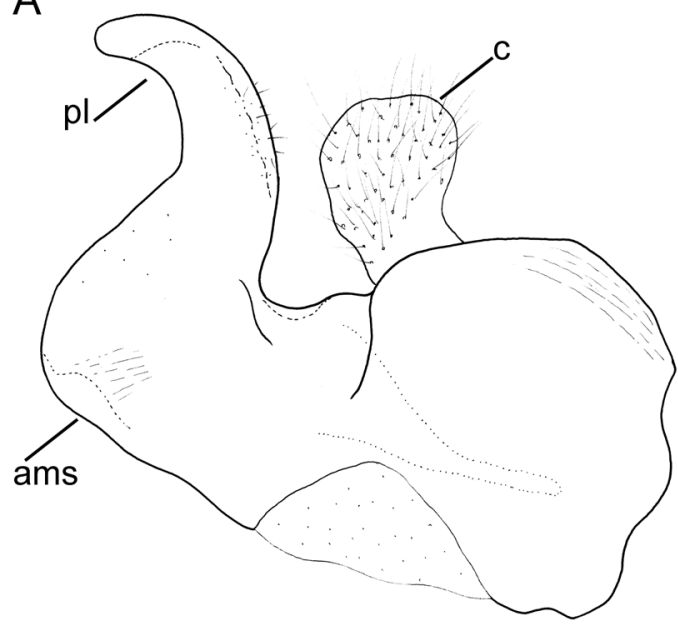

B

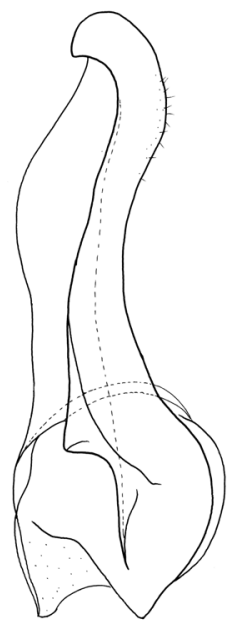

C

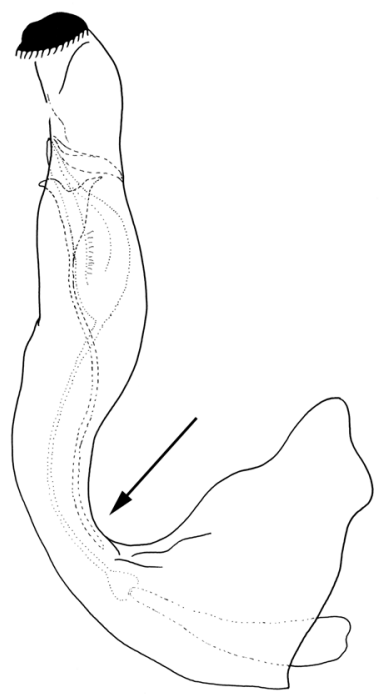

$E$

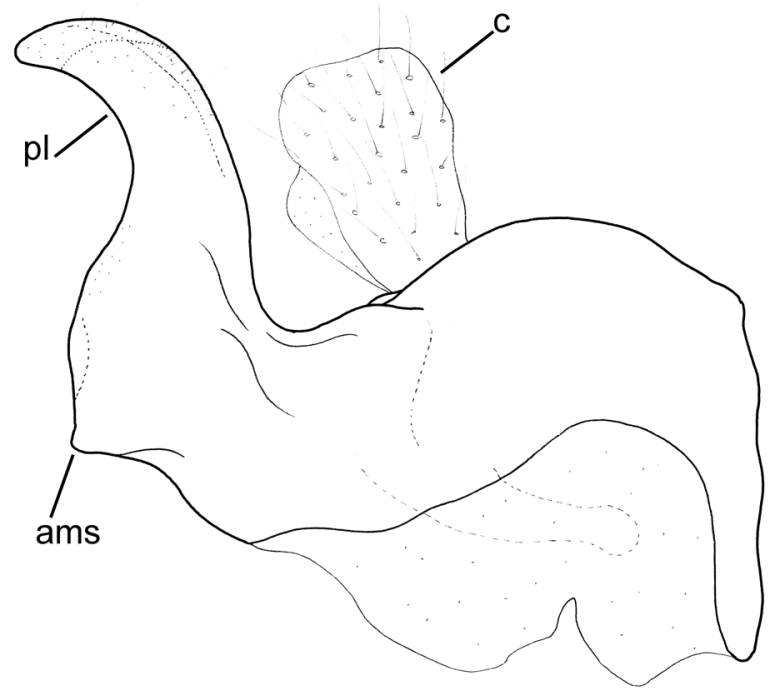

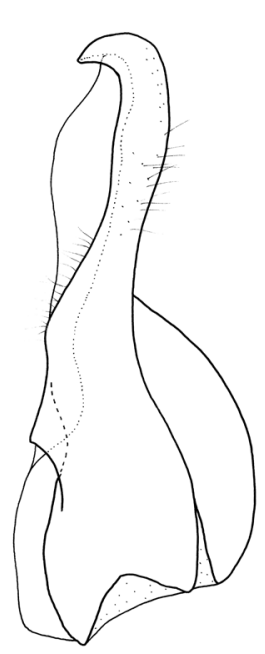

$\mathrm{F}$

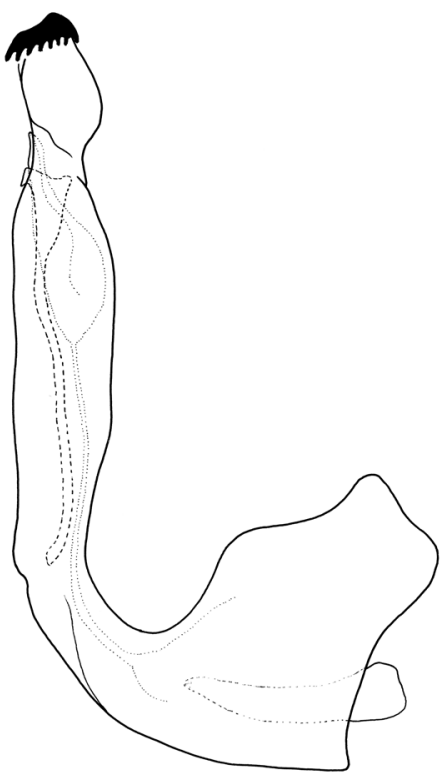

Fig. 7. Male genitalia. A-C. Merodon zebra Vujić \& Radenković sp. nov., paratype (NBCN; FSUNS ID 05174). D-F. M. vittatus Vujić \& Likov sp. nov., holotype (NBCN; FSUNS ID 04085). A, D. Epandrium, lateral view. B, E. Epandrium, ventral view. C, F. Hypandrium, lateral view. Abbreviations: ams = anterior margin of surstyle; $\mathrm{c}=$ cercus; $\mathrm{pl}=$ posterior lobe of surstyle. Scale bar $=0.25 \mathrm{~mm}$. 
AвDOMEn (Fig. 5E). Black, covered with yellow to whitish pile on lateral sides. Tergum 1 covered with short whitish to yellow pile; terga 2 and 3 medially with shorter black pile, except two medial fasciae with whitish pile and some whitish pile at posterior margin; anterior half of tergum 4 covered with short black pile, posterior half with longer yellowish pilosity, medially with two oblique fasciae covered with whitish pile. Sterna dark brown, covered with yellowish pile.

Genitalia (Figs 17, 18A). Posterior lobe of surstyle broad, bent, S-shaped, gradually narrowing toward tip (Fig. 17A, D: pl); anterior lobe of surstyle undeveloped (Fig. 17A, D); anterior margin of surstyle rounded in lateral view (Fig. 17A, D: ams); cercus square like (Fig. 17A, D: c). Hypandrium with theca medially distinctly narrowed (Fig. 17C, F). Lateral sclerite of aedeagus small (Fig. 18A: s).

Female (Figs 14D, 15D, 16D)

Similar to the male except for normal sexual dimorphism: Frons shiny, non pollinose, except along eye margins (Fig. 15D); tergum 4 with a pair of indistinct narrow oblique pollinose fasciae.

\section{Period of flight and distribution (Fig. 1)}

Merodon lotus sp. nov. occurs in RSA in the Ceres Mountain Fynbos Nature Reserve; the flight period is in November and December. This species appears in Mediterranean evergreen forest-hard-leaf scrub in the south of the African continent (Sayre et al. 2013).
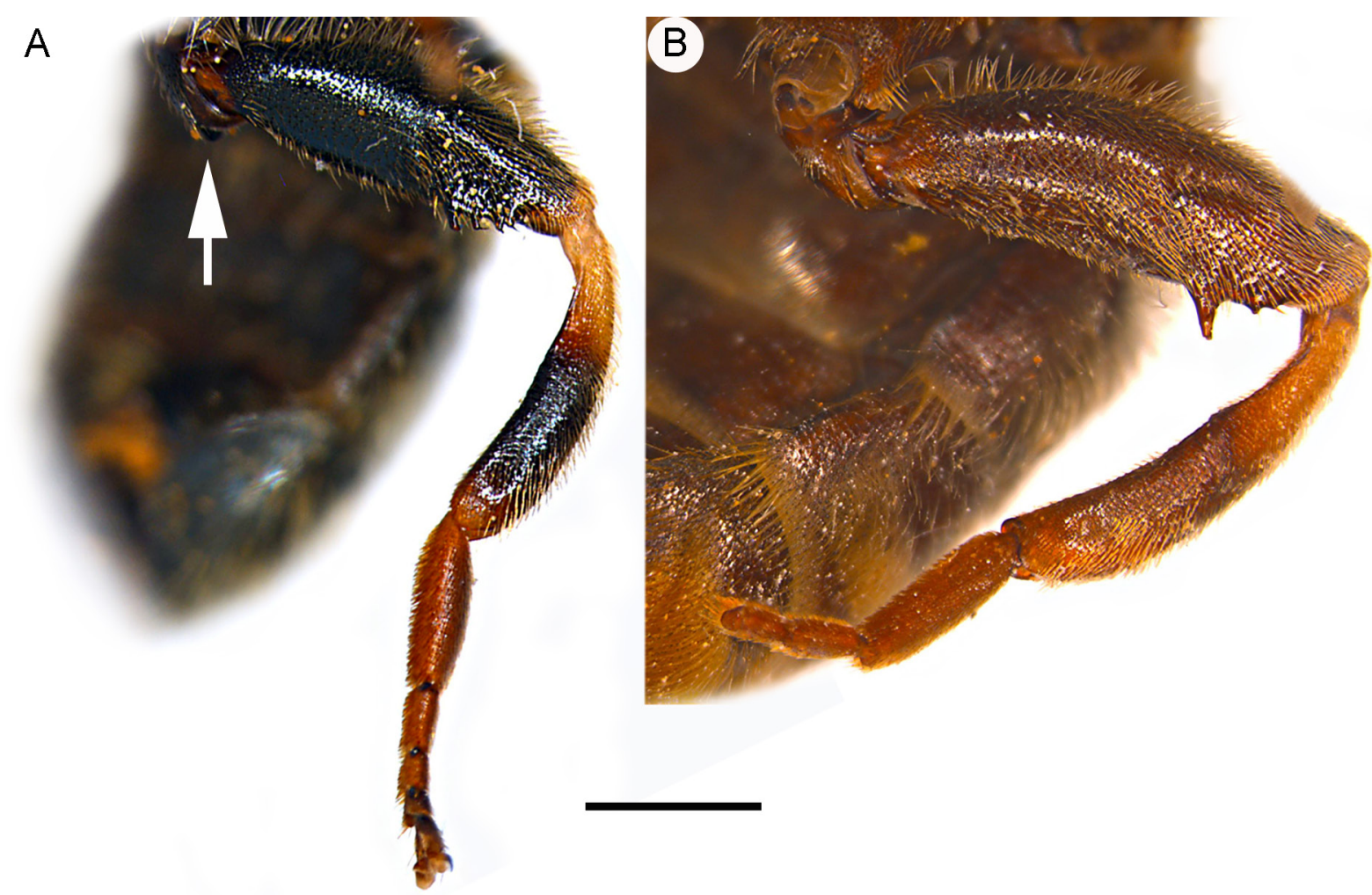

Fig. 8. Male metaleg, lateral view. A. Merodon funestus (Fabricius, 1794) (CWM; FSUNS 10347). B. M. vittatus Vujić \& Likov sp. nov., holotype (NBCN; FSUNS ID 04085). Calcar on metatrochanter marked with arrow. Scale bar $=2 \mathrm{~mm}$. 
VUJIĆ A. et al., Revision of the Merodon bombiformis species group

\section{Flower visited}

During our fieldwork, adults of $M$. lotus sp. nov. were observed as regular visitors of flowers of the genus Coleonema Bartl. \& H.L.Wendl. (Rutaceae) in Ceres (Fig. 25).

Merodon multifasciatus Curran, 1939

Figs 1, 4B, 5B, 6B, 9B, 10B, 11B, 12B, 13D-F, 14B, 15B, 16B, 18B, 21F-H, 25

Merodon multifasciatus Curran, 1939: 8 (type locality: Johannesburg, Republic of South Africa).

Merodon apimima Hull, 1944: 40-42 (type locality: Mulanje, Nyasaland, Republic of Malawi).

\section{Diagnosis}

Large (10-12 mm) and dark, striped species, with golden-yellow pile on the tip of the abdomen (Figs 5B, 14B). Scutum with conspicuous pollinose vittae (Figs 10B, 14B); metafemur with distinct apical dens on triangular lamina (Figs 6B, 16B); terga 3 and 4 with broad medial pollinose fasciae (Figs 5B, 14B). Similar to M. vittatus Vujić \& Likov sp. nov. from which differs in male genitalia with wider posterior lobe of surstyle, slightly curved, almost parallel sided, with rounded apex (Fig. 13D: pl), while male genitalia in M. vittatus sp. nov. with longer and narrower, S-shaped posterior lobe of surstyle, gradually narrowing toward tip (Fig. 7D: pl).

\section{Material examined}

Holotype

REPUBLIC OF SOUTH AFRICA • 1 q; Johannesburg; 26.284 S, 27.982 E; 6000 ft; Mar. 1899; J.P. Cregoe leg.; AMNH.

\section{Additional material}

REPUBLIC OF MALAWI 1 $1 \hat{\partial}$, holotype of Merodon apimima; Mulanje, Nyasaland; $16.015^{\circ} \mathrm{S}$, $35.521^{\circ}$ E; 22 Apr. 1913; S.A. Neave leg.; BMNH 1 q, paratype of Merodon apimima; same collection data as for preceding; 21 Oct. 1913; labelled as allotype; BMNH $\bullet 2 \hat{\jmath}$, paratypes of Merodon apimima; same collection data as for preceding; 18-30 Apr. 1913; BMNH $\bullet 1$, paratype of Merodon apimima; same collection data as for preceding; FSUNS ID 04086; NBCN $\bullet 2 \hat{\jmath}$, paratypes of Merodon apimima; same collection data as for preceding; 2 Jun. 1913; BMNH 11 , paratype of Merodon apimima; same collection data as for preceding; FSUNS ID 04355; BMNH.

\section{Additional non type material}

DEMOCRATIC REPUBLIC OF THE CONGO • $1{ }^{\lambda}$; Lualaba River; $7.023^{\circ}$ S, $27.046^{\circ}$ E; $2500-$ 4000 ft; 21 Apr. 1907; S.A. Neave leg.; FSUNS ID 03909; NBCN LML-05-3.

REPUBLIC OF SOUTH AFRICA • 1 ô; Modimolle (former Nylstroom); $24.683^{\circ} \mathrm{S}, 28.416^{\circ} \mathrm{E} ; 17 \mathrm{Feb}$. 1908; unknown leg.; reared from Gladiolus bulb; USNM • 1 ते; Transvaal, $5 \mathrm{mi}(8 \mathrm{~km}) \mathrm{W}$ of Warmbad; 24-25 Feb. 1968; K.V. Krombein leg.; FSUNS ID 04512; USNM ENT 00036564, USNM 2052372 • $1 \partial^{\lambda}$; Transvaal, Fortuna Trail, S of Barberton, N slope; $26.009^{\circ} \mathrm{S}, 31.048^{\circ} \mathrm{E}$; 8 Apr. 1985; J.G.H. Londt leg.; bushveld; FSUNS ID 04284; NMSA • 1 q; Mpumalanga, Emgwenya (Waterval Boven), near Elandsrivier; $25.634^{\circ} \mathrm{S}, 30.326^{\circ}$ E; 6 Apr. 2018; Sanja Vujić leg.; FSUNS ZA5_242; FSUNS • 1 \%; same collection data as for preceding; L,S. Forcada leg.; FSUNS ZA5_243; FSUNS.

\section{Redescription}

Original descriptions are insufficient, without any illustrations and based on a single female from South Africa (Curran 1939) and male and female types (Hull 1944). 
A

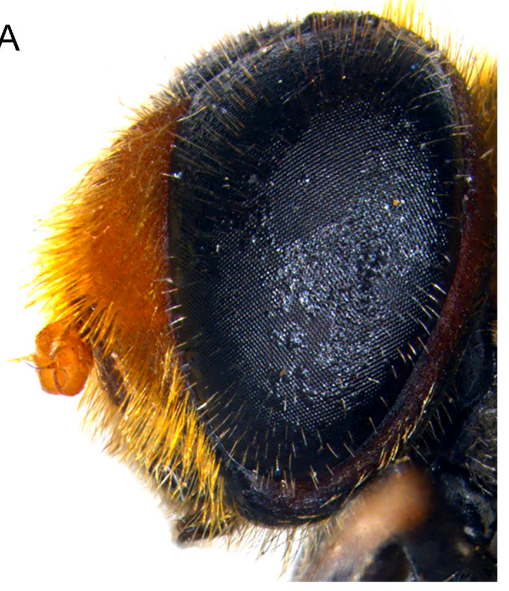

C

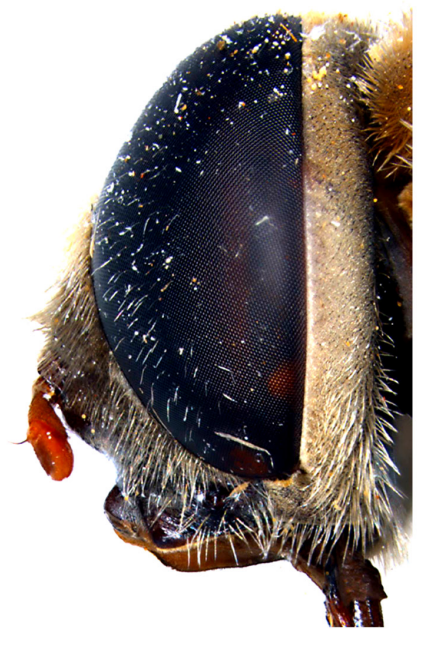

$E$

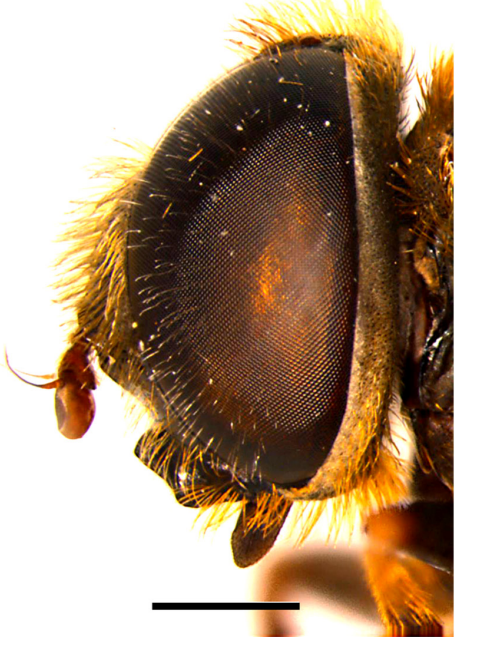

B

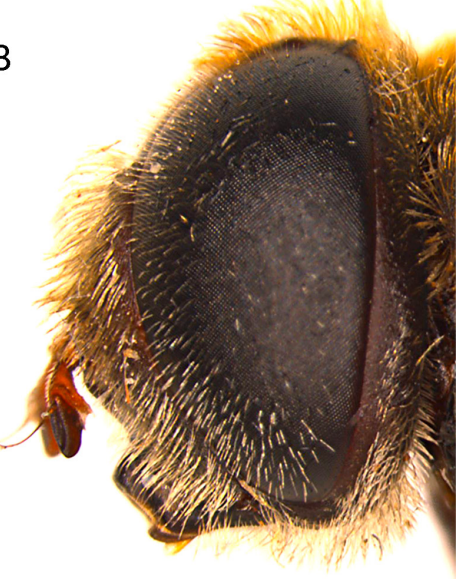

D

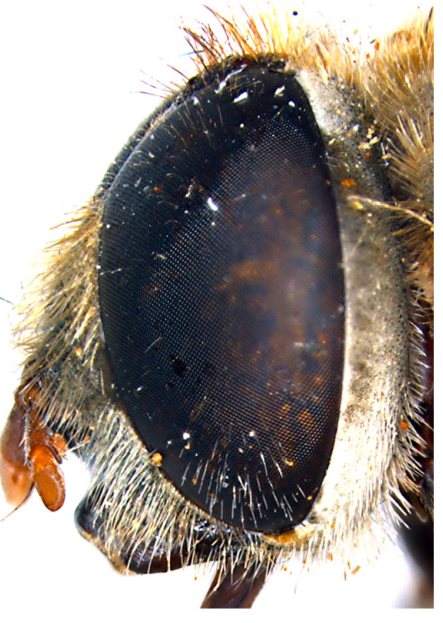

$\mathrm{F}$

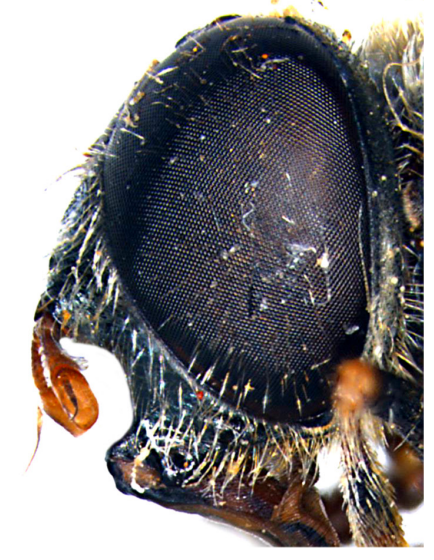

Fig. 9. Male head, lateral view. A. Merodon bombiformis Hull, 1944 (FSUNS; FSUNS ZA2_079). B. M. multifasciatus Curran, 1939 (USNM 2052372; FSUNS ID 04512). C. M. vittatus Vujić \& Likov sp. nov., holotype (NBCN; FSUNS ID 04085). D. M. zebra Vujić \& Radenković sp. nov., holotype (RMCA; FSUNS ID 25089). E. M. lotus Vujić \& Radenković sp. nov., paratype (FSUNS; FSUNS ID ZA6_066). F. M. nasicus Bezzi, 1915 (TAUI; FSUNS ID 04972). Scale bar $=2$ mm. 


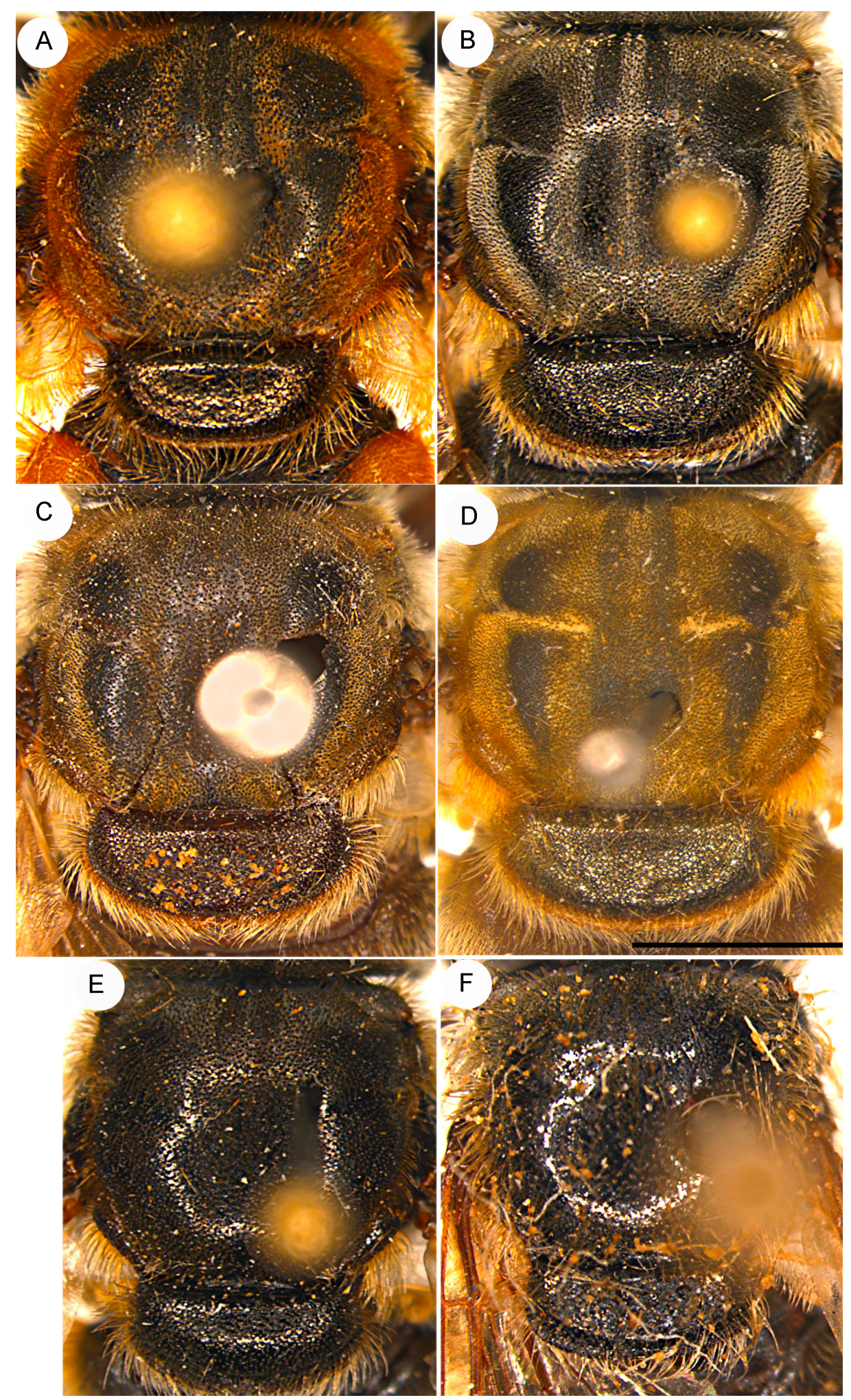

Fig. 10. Mesonotum, dorsal view. A. Merodon bombiformis Hull, 1944 (FSUNS; FSUNS ZA2_079). B. M. multifasciatus Curran, 1939 (FSUNS; FSUNS ID ZA5_243). C. M. vittatus Vujić \& Likov sp. nov., holotype (NBCN; FSUNS ID 04085). D. M. zebra Vujić \& Radenković sp. nov., paratype (RMCA; FSUNS ID 25090). E. M. lotus Vujić \& Radenković sp. nov., paratype (FSUNS; FSUNS ID ZA6_066). F. M. nasicus Bezzi, 1915 (TAUI; FSUNS ID 04972). A, C, E-F. Male. B, D. Female. Scale bar $=\overline{2}$ mm. 

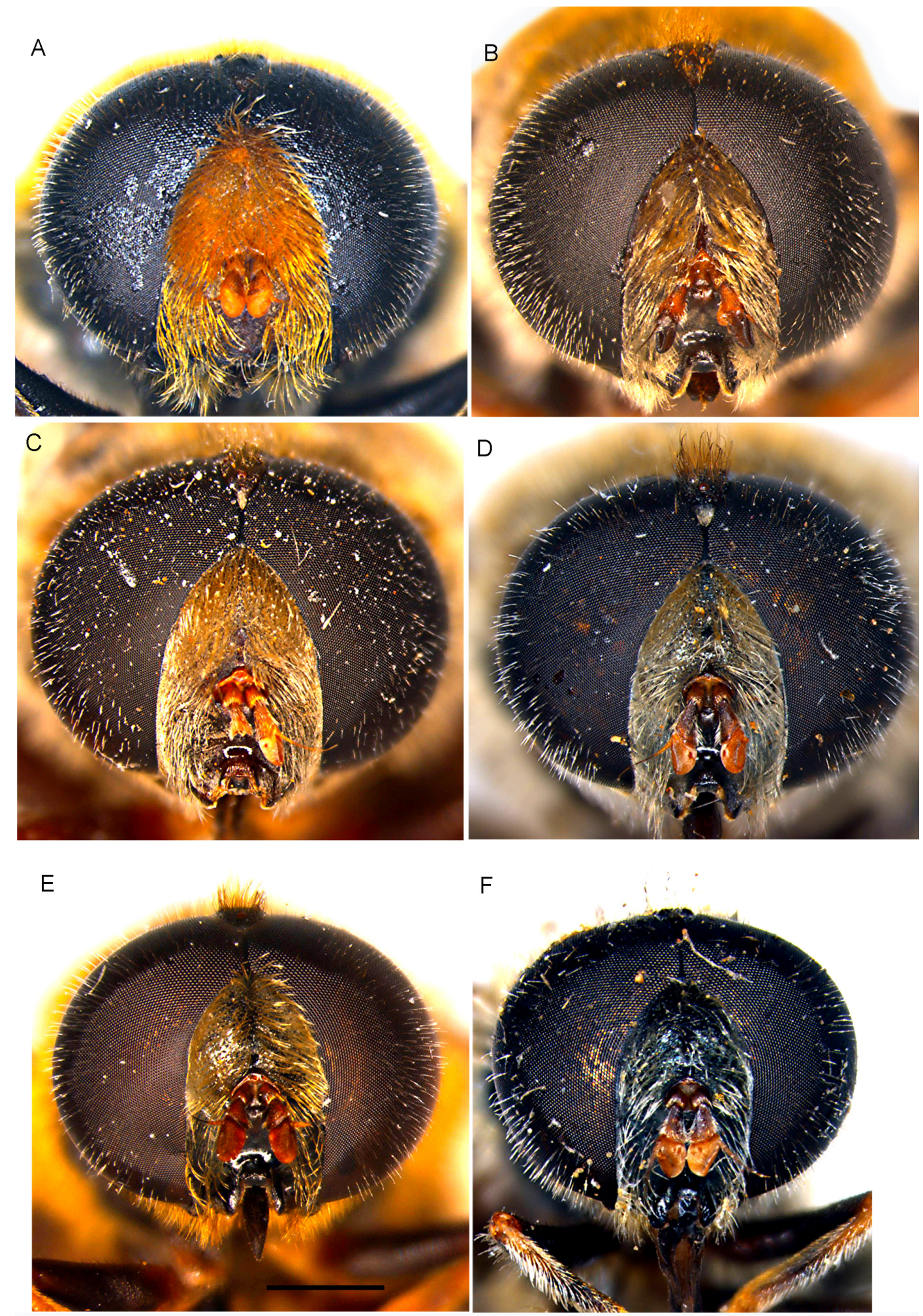

Fig. 11. Male head, frontal view. A. Merodon bombiformis Hull, 1944 (FSUNS; FSUNS ZA2_079). B. M. multifasciatus Curran, 1939 (USNM 2052372; FSUNS ID 04512). C. M. vittatus Vujić \& Likov sp. nov., holotype (NBCN; FSUNS ID 04085). D. M. zebra Vujić \& Radenković sp. nov., holotype (RMCA; FSUNS ID 25089). E. M. lotus Vujić \& Radenković sp. nov., paratype (FSUNS; FSUNS ID ZA6_066). F. M. nasicus Bezzi, 1915 (TAUI; FSUNS ID 04972). Scale bar $=2 \mathrm{~mm}$. 
Length: large species, body 10-12 $\mathrm{mm}$, wing $8-9 \mathrm{~mm}(\mathrm{n}=5)$.

Male

Head (Figs 4B, 9B, 11B, 12B). Antenna (Fig. 4B) reddish-brown; pedicel elongated, approximately as long as basoflagellomere (relation scape : pedicel : basoflagellomere $=1.0: 2.5: 2.5$ ); basoflagellomere concave dorsally, with acute apex; arista light brown to dark brown, thickened basally, 1.6 times as long as basoflagellomere. Face from black to dark brown, covered with gray pollinosity and whitish pile, except on bare medial vitta that occupies $1 / 4$ width of face. Oral margin protruded (Fig. 9B). Frons from black to reddish, inflated, covered with gray pollinosity and whitish pile. Vertical triangle and isosceles (Fig. 12B), usually dark brown, predominantly covered with a long, yellow pile. Ocellar triangle equilateral. Eye pile dense, gray, slightly longer than scape. Eye contiguity about 15-18 ommatidia long. Occiput reddish, pollinose, covered with yellow pile.

Thorax (Figs 6B, 10B). Mesonotum black, except postpronotum and posterior margin of scutellum brown-reddish, covered with reddish-yellow pile; scutum with five pollinose longitudinal vittae (as on Fig. 10B). Pleuron black to dark brown, covered with gray pollinosity and the following parts with whitish to yellow pile: anterior part of proepimeron, posterior part of anterior anepisternum, most of the posterior anepisternum except anterior end, antero-ventral and postero-dorsal part of katepisternum, anepimeron, and metasternum. Wing hyaline, with dense microtrichia and yellow to brown veins. Calypter pale yellow. Halter with yellow pedicel and capitulum. All three femora dark brown to black; tibiae mostly reddish, medially black to dark brown; tarsi reddish (Fig. 6B). Metatrochanter without calcar. Metafemur moderately thickened, with less developed apicoventral triangular lamina, more or less dentate, the apical dens is distinct (Fig. 6B). Pile on legs predominantly whitish to yellow.

AвDOMEn (Fig. 5B). Black to dark brown. Tergum 1 black, usually pollinosed, covered with short whitish to yellow pile; tergum 2 with pollinose posterior margin and narrow medial fascia (in some specimens interrupted in the middle) covered with whitish pile except posterior half of tergum (between pollinose fasciae) covered with short black pilosity; terga 3 and 4 with pollinose posterior margin and broad medial pollinose fasciae (Fig. 5B); anterior half of tergum 3 covered with short black pile; tergum 4 covered with long golden-yellow pilosity (Fig. 5B). Sterna reddish-brown, covered with white or yellow pile.

Genitalia (Figs 13D-F, 18B). Posterior lobe of surstyle broad, slightly curved, almost parallel sided, with rounded apex (Fig. 13D: pl); anterior lobe of surstyle undeveloped (Fig. 13D); anterior margin of surstyle angular in lateral view (Fig. 13D: ams); cercus square like (Fig. 13D: c). Hypandrium with theca medially distinctly narrowed (Fig. 13F). Lateral sclerite of aedeagus small (Fig. 18B: s).

Female (Figs 10B, 14B, 15B, 16B)

Similar to the male except for normal sexual dimorphism: metafemur usually with small apical dens on triangular lamina (Fig. 16B); frons with non pollinose medial vitta (Fig. 15B); vertex predominantly covered with black pile.

\section{Remarks}

Hull (1944) described Merodon apimima "related to multifasciatus Curran in type of femoral armament and abdominal fascia; distinct in the black front, yellow pilose vertex and, brown humeri, absence of red on second abdominal segment, beside other details of pattern" based on six males and one female. All these characters mentioned in original description can be regarded as intraspecific variability of M. multifasciatus. Based on our study of the type material of both species, we regard M. apimima as a junior synonym of $M$. multifasciatus. 
A
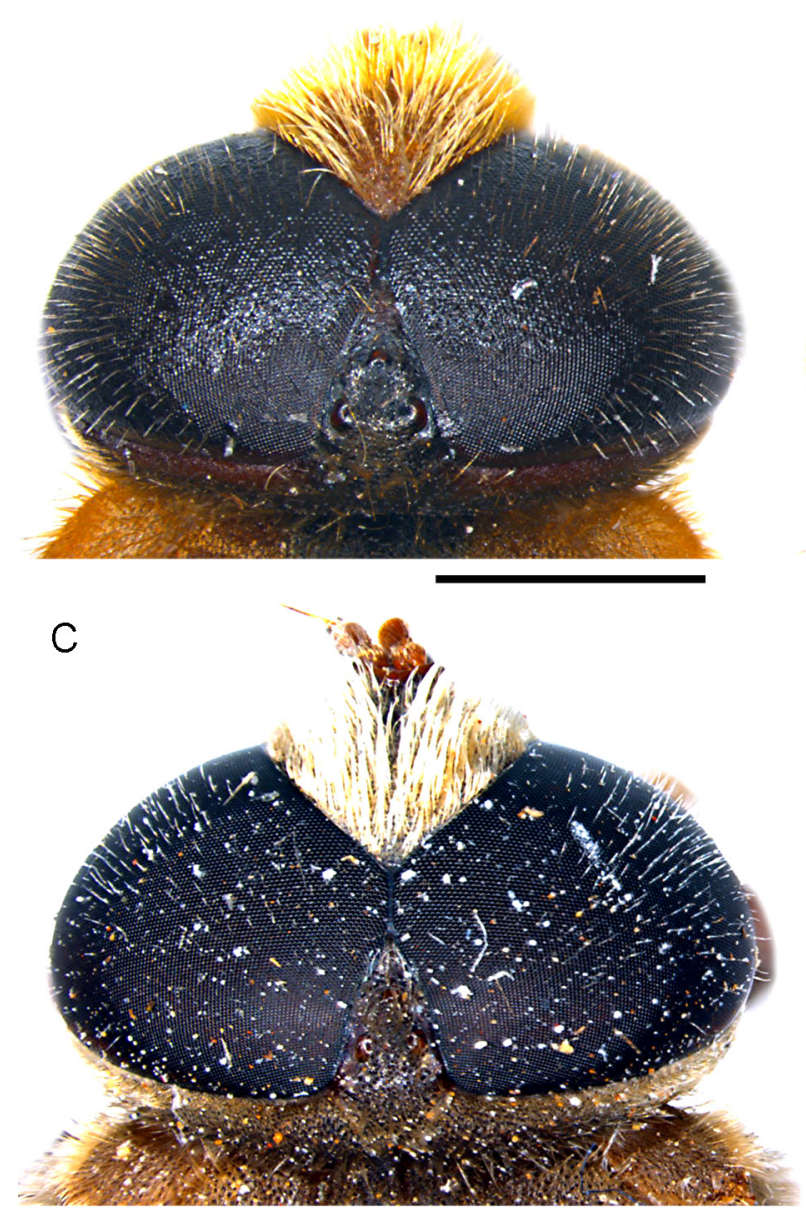

$E$

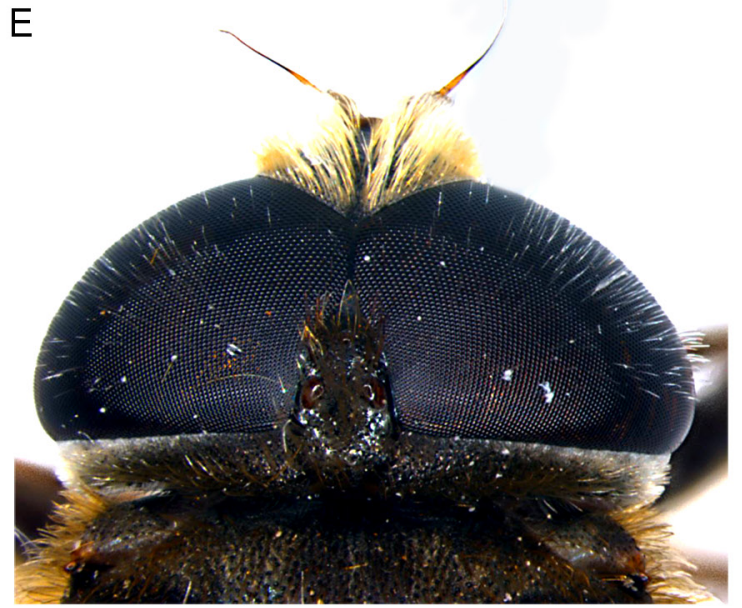

$\mathrm{B}$

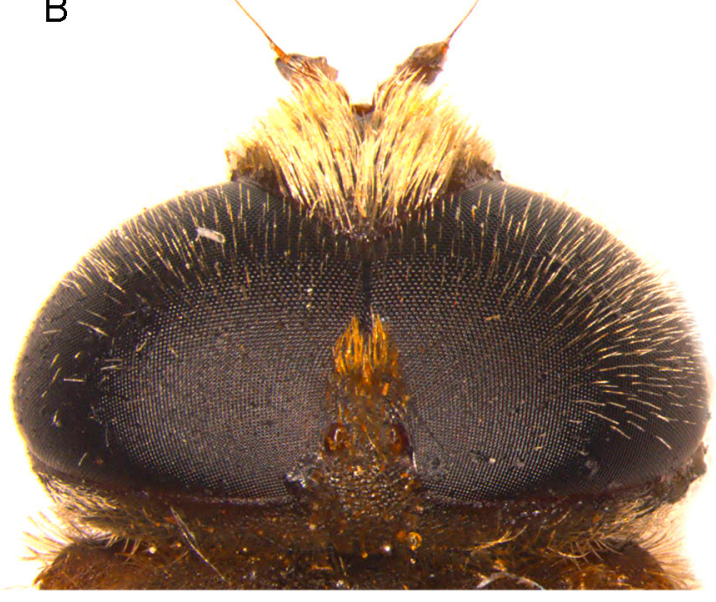

$\mathrm{D}$

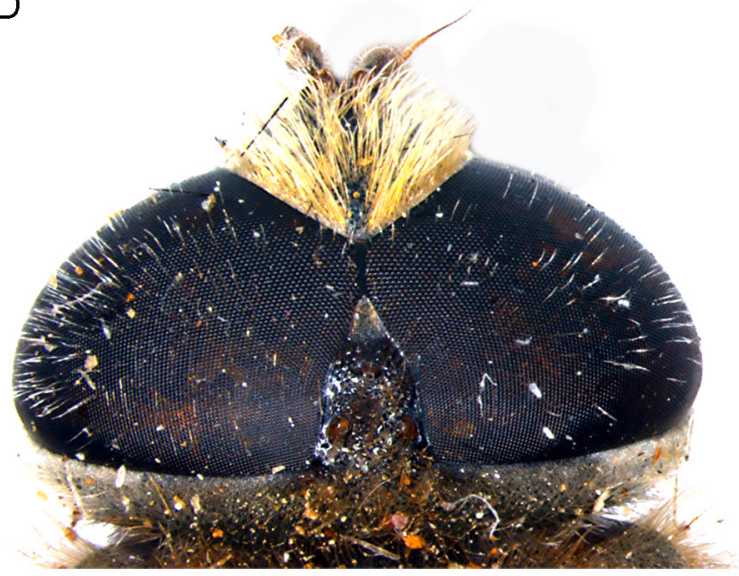

$\mathrm{F}$

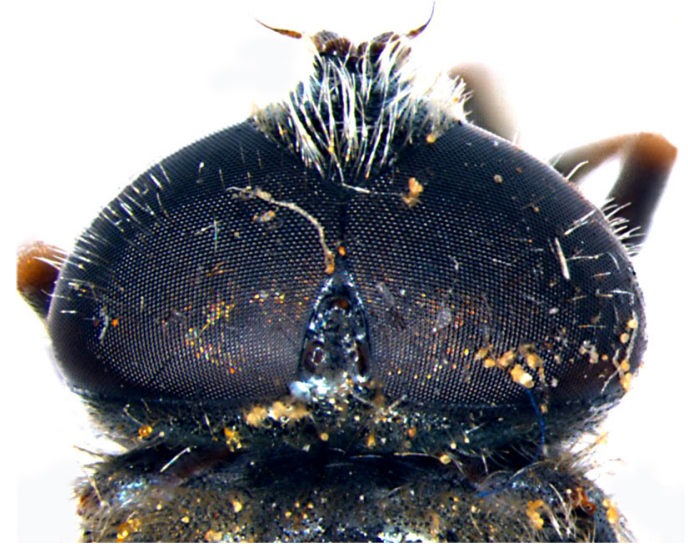

Fig. 12. Male head, dorsal view. A. Merodon bombiformis Hull, 1944 (FSUNS; FSUNS ZA2_079). B. M. multifasciatus Curran, 1939 (USNM 2052372; FSUNS ID 04512). C. M. vittatus Vujić \& Likov sp. nov., holotype (NBCN; FSUNS ID 04085). D. M. zebra Vujić \& Radenković sp. nov., holotype (RMCA; FSUNS ID 25089). E. M. lotus Vujić \& Radenković sp. nov., paratype (FSUNS; FSUNS ID ZA6_066). F. M. nasicus Bezzi, 1915 (TAUI; FSUNS ID 04972). Scale bar $=2 \mathrm{~mm}$. 
In the original description of species, Hull (1944) did not state the etymology of the species name, so we cannot conclude whether he treated "apimima" as a noun or an adjective. According to the International Code of Zoological Nomenclature (Article 31.2.2) (International Commission on Zoological Nomenclature 1999) if the author does not indicate whether the species name is a noun or an adjective, as in this case, it has to be treated as a noun. When the species name is a noun simple or compound, in apposition, it does not need to agree in gender with the generic name, therefore the original spelling has to be retained (Article 34.2.1). In some references (Smith \& Vockeroth 1980; Dirickx 1988) M. apimimus is used because authors considered "apimima" to be an adjective. But in Systema Dipterorum (Evenhuis \& Pape 2020), which is the most comprehensive database related to the Syrphidae, the original spelling of M. apimima is applied.

\section{Period of flight and distribution (Fig. 1)}

This species occurs in Malawi, the Republic of South Africa and the Democratic Republic of Congo. On the basis of our data, the flight period was found to be throughout the whole year. Merodon multifasciatus was found in deciduous forest-woodland savanna and brush-grass savanna.

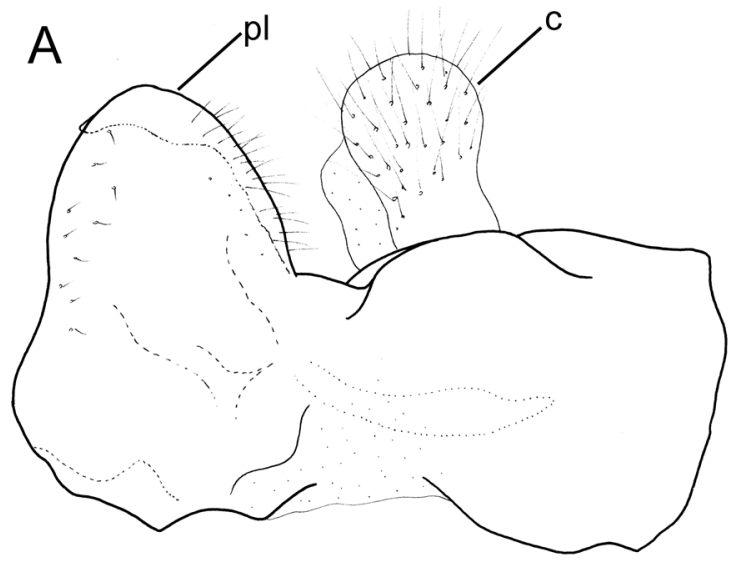

B

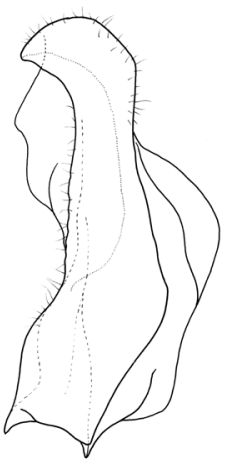

$\mathrm{D}$

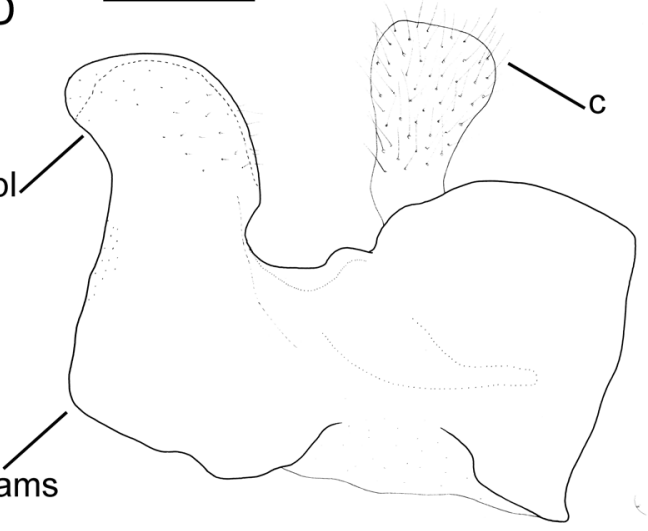

E
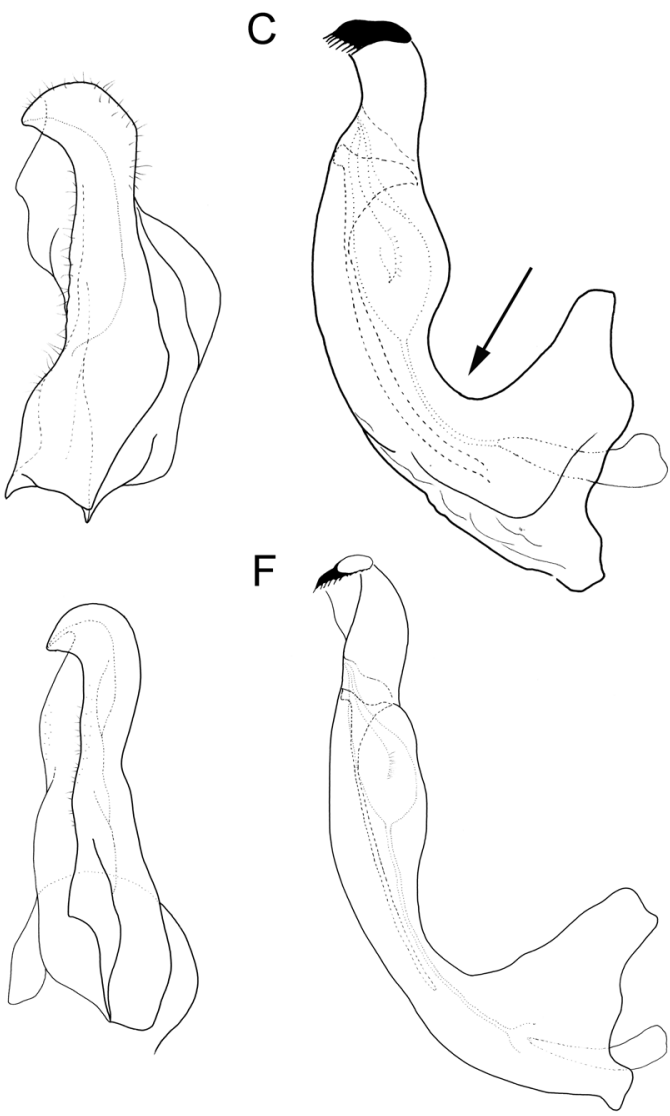

Fig. 13. Male genitalia. A-C. Merodon bombiformis Hull, 1944 (NMSA; FSUNS ID 04277). D-F. M. multifasciatus Curran, 1939 (NMSA; FSUNS ID 04284). A, D. Epandrium, lateral view. B, E. Epandrium, ventral view. C, F. Hypandrium, lateral view. Abbreviations: ams = anterior margin of surstyle; $\mathrm{c}=$ cercus; $\mathrm{pl}=$ posterior lobe of surstyle. Medially narrowed hypandrium marked with arrow. Scale bar $=0.5 \mathrm{~mm}$. 


\section{Larval biology}

The material from Nylstroom (17 Feb. 1908) was bred from bulbs of Gladiolus (immature stages undescribed). Specimens collected in Emgwenya (Waterval Boven) (6 Apr. 2018) were found on flowers of different plant species inside a small spot $(20 \times 40 \mathrm{~m})$ with the natural population of Gladiolus sericeovillosus Hook., along a small periodical spring. It is a strong indication that this plant is the primary host for larvae of M. multifasciatus.

\section{Flower visited}

During our fileldwork, adults of $M$. multifasciatus were observed visiting flowers of Nidorella auriculata DC. in Emgwenya (Waterval Boven) (Fig. 26).

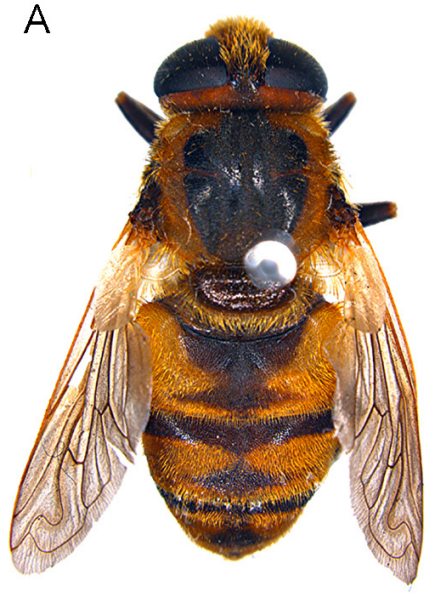

D

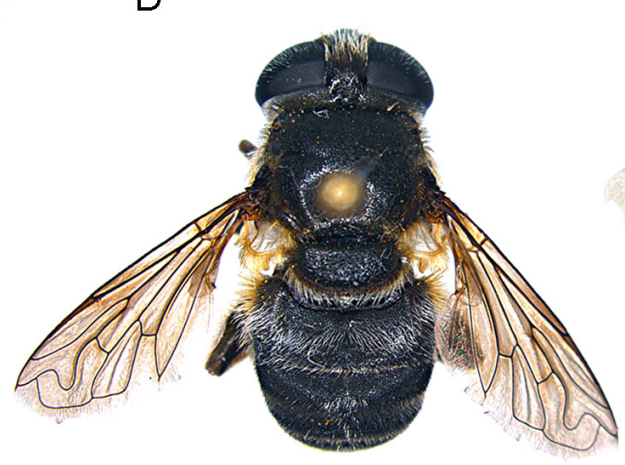

B

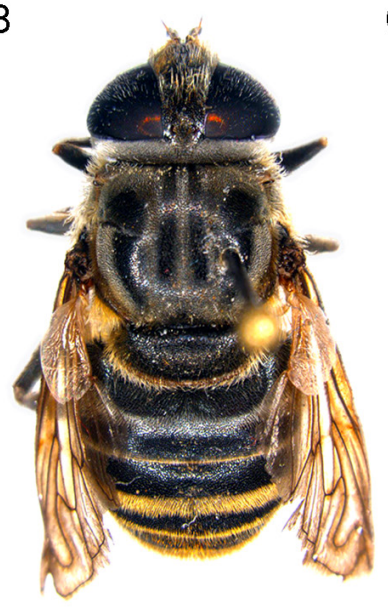

C

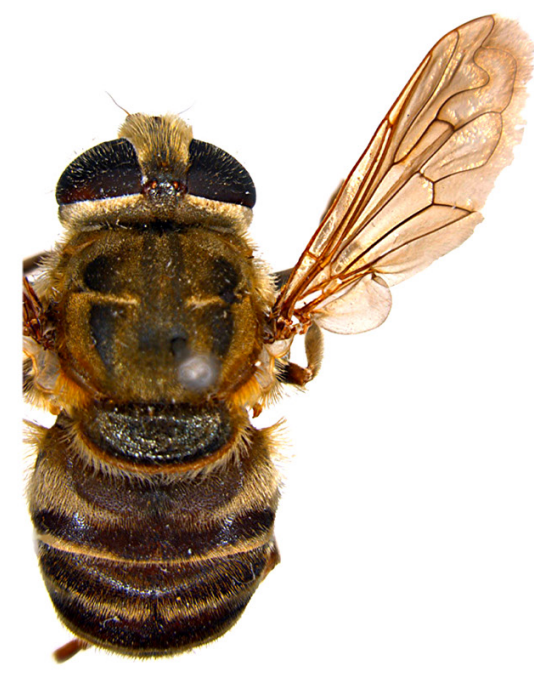

$E$

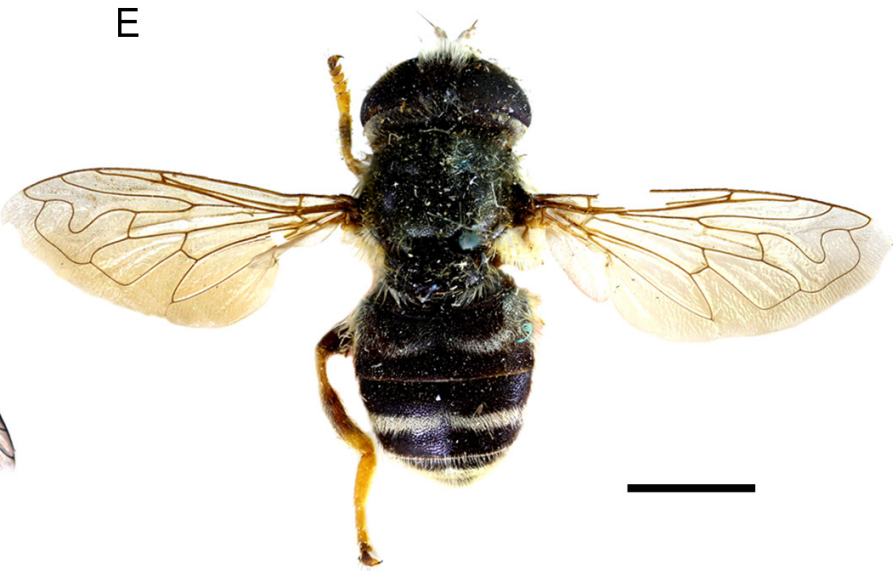

Fig. 14. Habitus of the female, dorsal view. A. Merodon bombiformis Hull, 1944 (NMSA; FSUNS ID 04278). B. M. multifasciatus Curran, 1939 (FSUNS; FSUNS ID ZA5_243). C. M. zebra Vujić \& Radenković sp. nov., paratype (RMCA; FSUNS ID 25090). D. M. lotus Vujić \& Radenković sp. nov., paratype (NMSA; FSUNS ID ZA6_067). E. M. nasicus Bezzi, 1915, lectotype (BMNH NHMUK010369943). Scale bar $=2 \mathrm{~mm}$. 
Merodon nasicus Bezzi, 1915

Figs 1, 4F, 5F, 6F, 9F, 10F, 11F, 12F, 14E, 15E, 16E, 18D, 19D-F, 20, 23A-B

Merodon nasicus Bezzi, 1915: 102 (type locality: Kenya, Njoro).

\section{Diagnosis}

Small $(6-7 \mathrm{~mm})$, black species with narrow, white pollinose, tergal fasciae. Oral margin distinctly protruded (Fig. 9F); face and frons without pollinosity, except narrow vitta along eye margins; terga 2-4 with medial pollinose fasciae (Figs 5F, 14E); tergum 4 covered with whitish to yellowish pile; male genitalia with S-shaped posterior lobe of surstyle, gradually narrowing toward tip (Fig. 19D: pl), anterior margin of surstyle rounded in lateral view (Fig. 19D: ams). Differs from Merodon bombiformis, M. vittatus sp. nov., M. multifasciatus and M. zebra Vujić \& Radenković sp. nov. by absence of distinct pollinose vittae on scutum (conspicuous in these four species); narrow medial pollinose fasciae on terga 3 and 4 (broad in M. vittatus sp. nov., M. multifasciatus and M. zebra sp. nov.) and male genitalia with moderately broad posterior lobe of surstylus, narrower than in M. multifasciatus, but broader than in M. vittatus sp. nov. and M. zebra sp. nov. (Figs 7A, D: pl, 19D: pl). Similar to M. lotus sp. nov. from which differs in presence of distinct medial pollinose fasciae on terga 2 and 3 (absent in males and indistinct in female of M. lotus sp. nov.) and more protruded oral margin (Fig. 9E-F).

\section{Material examined}

Lectotype (designated here by A. Vujić)

KENYA • 1 क; Njoro, about $140 \mathrm{~km} \mathrm{NW}$ of Nairobi; $0.37^{\circ} \mathrm{S}, 35.917^{\circ} \mathrm{E} ; 2322 \mathrm{~m}$ a.s.l.; date unknown; A.J. Cholmley leg.; BMNH NHMUK010369943.

Bezzi published the Syrphidae of the Ethiopian Region with the descriptions of two new species, Merodon planifacies and M. melanocerus (Bezzi 1915). In the same work, he presented diagnostic characters for, and notes on, the third Ethiopian species, M. nasicus, referring to 1914 as the year of its description (Bezzi 1915: 102), based on material from Eritrea in the collections of the Hungarian Museum. Bezzi (1915) also stated that there was a single female specimen of this species from Njoro, in British East Africa. Subsequently, in the Catalogue of the Diptera of the Afrotropical Region (Smith \& Vockeroth 1980), and in the Diptera Database (Evenhuis \& Pape 2020), this Njoro specimen was erroneously considered as the holotype. Shortly after his 1915 monograph, Bezzi published a description of Lampetia (Merodon) nasica (Bezzi 1921), based on a male and female from Eritrea in the collection of the Hungarian Museum. This is actually the unpublished description from 1914 (Bezzi 1915). However, based on International Code of Zoological Nomenclature (International Commission on Zoological Nomenclature 1999), the oldest available name for a taxon is its valid name, therefore the publication with the first appearance of the name of the taxon (Bezzi 1915) should be considered as valid. Following from this conclusion, in addition to specimens from Eritrea, cited in both publications (Bezzi 1915, 1921), one female specimen from Kenya (Bezzi 1915), deposited in BMNH, also belongs to the syntype series. The Diptera collection in the Hungarian National Museum in Budapest, including the syntypes from Eritrea, was destroyed by a fire in 1956. However, in order to stabilize nomenclature should additional specimens of the type series be found, we designate the specimen from BMNH as lectotype.

\section{Additional material}

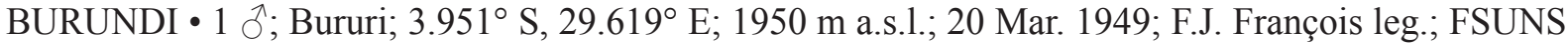
ID 21976; RBINS.

ETHIOPIA • 1 \% ; Welo Gondi, $60 \mathrm{~km} \mathrm{SW}$ Lalibela; $7.779^{\circ} \mathrm{N}, 35.458^{\circ} \mathrm{E} ; 2485 \mathrm{~m}$ a.s.1.; 9 Oct. 2005; A. Freidberg leg.; FSUNS ID 04972; TAUI. 
ERITREA • 1 \%; Adi Keyh (on eastern slope of Ethiopian highlands); $14.816^{\circ} \mathrm{N}, 39.383^{\circ} \mathrm{E} ; 2442 \mathrm{~m}$ a.s.1.; Sep. 1902; Dr A. Andreini leg.; LSF.

\section{Redescription}

Original description without any illustrations was based on single male and female (Bezzi 1921).

Length: small sized species, body 6-7 mm, wing 5-6 mm ( $\mathrm{n}=2)$.

\section{Male}

HeAD (Figs 4F, 9F, 11F, 12F). Antenna (Fig. 4F) reddish-brown; pedicel elongated, approximately as long as basoflagellomere (relation scape:pedicel: basoflagellomere $=1.0: 3.0: 3.0$ ); basoflagellomere concave dorsally, with acute apex; arista reddish-brown, thickened basally, 1.3 times as long as basoflagellomere. Face black, without pollinosity, except along eye margins and covered with whitish to yellowish pile, except on bare medial vitta that occupies $1 / 2$ width of face. Oral margin strongly protruded (Fig. 9F). Frons black, without gray pollinosity, except eye margins, covered with whitish to yellowish pile. Vertical triangle isosceles (Fig. 12F), usually dark brown, predominantly covered with a long, yellow pile. Ocellar triangle equilateral. Eye pile black in upper half and gray in lower, as long as scape. Eye contiguity about $15-18$ ommatidia long. Occiput black, partly pollinose, covered with yellowish to whitish pile.

Thorax (Fig. 6F). Mesonotum dark brown, covered with light yellowish to yellowish pile; scutum without or with indistinct pollinose longitudinal vittae; postpronotum and anterior margin of scutum with sparse gray pollinosity. Pleuron dark brown, covered with gray pollinosity and the following parts with whitish to yellow pile: anterior part of proepimeron, posterior part of anterior anepisternum, most of the posterior anepisternum except anterior end, antero-ventral and postero-dorsal part of katepisternum, anepimeron, metasternum. Wing hyaline, mostly covered with microtrichia; veins yellow to brown. Calypter pale yellow. Halter with yellow pedicel and capitulum. All three femora dark brown to black, except reddish apex; tibiae mostly reddish, medially dark brown; basal three tarsomeres yellowish to reddish, the rest blackish (Fig. 6F). Metatrochanter without calcar. Metafemur moderately thickened, with less developed apicoventral triangular lamina, more or less dentate, the apical dens is distinct (Fig. 6F). Pile on legs predominantly whitish to yellow.

AвDOMEn (Fig. 5F). Dark brown with yellow to whitish-yellow pile on lateral margins. Tergum 1 usually with pollinosity at least laterally, covered with short pile; tergum 2 with narrow medial fascia interrupted in the middle, covered with whitish pile; tergum 2 medially mostly covered with short black pilosity; terga 3 and 4 with or without narrow pollinose posterior margin and distinct medial fascia interrupted in the middle; tergum 3 covered with short black pile medially; tergum 4 covered with longer whitish to yellowish pilosity, except few black pile in anterior half. Brown sterna pollinose, covered with white or yellow pile.

Genitalia (Figs 18D, 19D-F). Posterior lobe of surstyle moderately broad, bent, S-shaped, gradually narrowing toward tip (Fig. 19D: pl); anterior lobe of surstyle undeveloped (Fig. 19D); anterior margin of surstyle rounded in lateral view (Fig. 19D: ams); cercus square like (Fig. 19D: c). Hypandrium with theca medially distinctly narrowed (Fig. 19F). Lateral sclerite of aedeagus small, pointed (Fig. 18D: s).

Female (Figs 14E, 15E, 16E, 20)

Similar to the male except for normal sexual dimorphism: metafemur usually with very small apical dens on triangular lamina (Figs 16E, 20B); frons with non pollinose medial vitta (Fig. 15E); vertex shiny, non pollinose, mostly covered with black pile. 

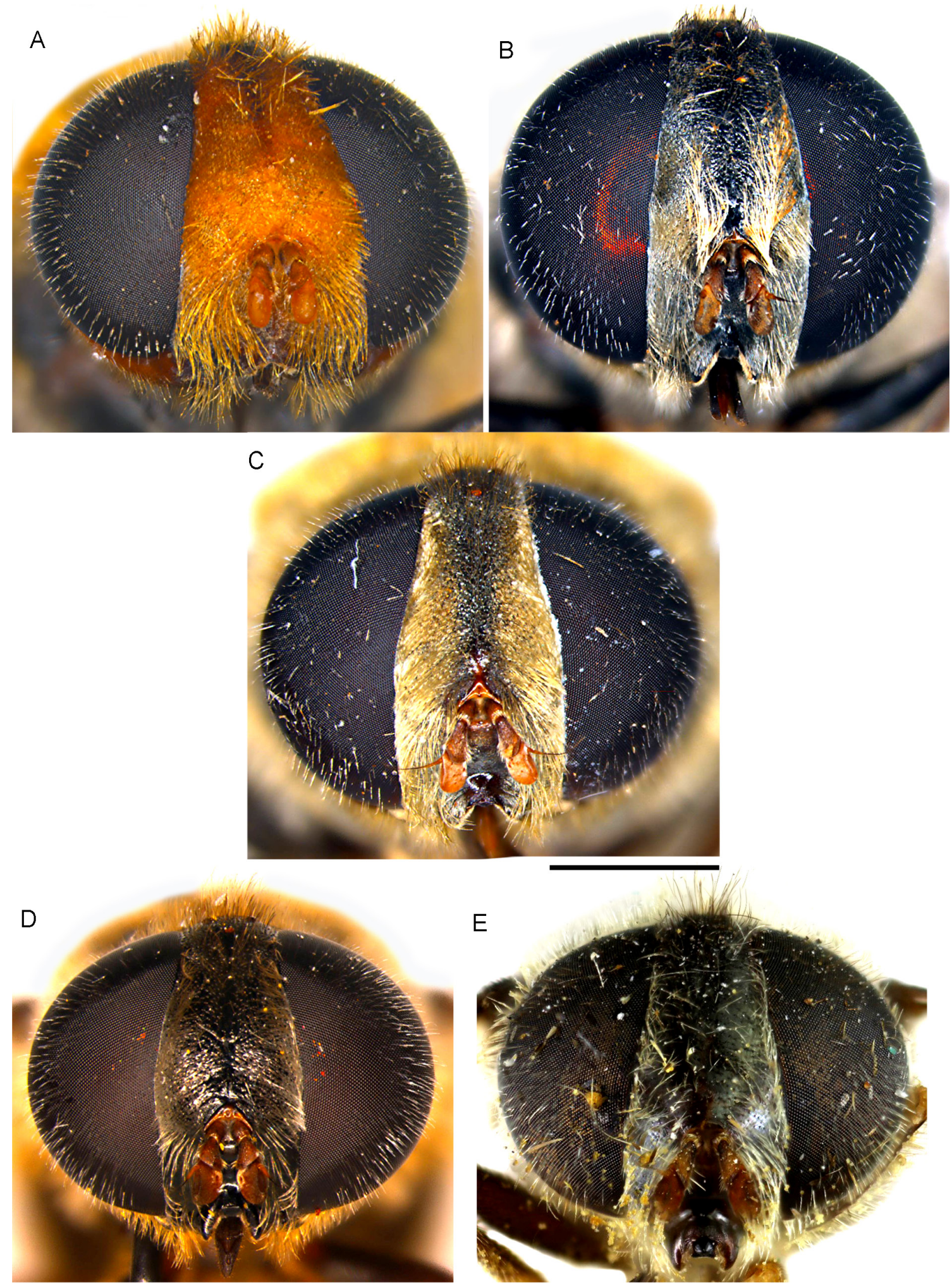

Fig. 15. Female head, frontal view. A. Merodon bombiformis Hull, 1944 (NMSA; FSUNS ID 04278). B. M. multifasciatus Curran, 1939 (FSUNS; FSUNS ID ZA5 243). C. M. zebra Vujić \& Radenković sp. nov., paratype (RMCA; FSUNS ID 25090). D. M. lotus Vujić \& Radenković sp. nov., paratype (NMSA; FSUNS ID ZA6_067). E. M. nasicus Bezzi, 1915, lectotype (BMNH; NHMUK010369943). Scale bar $=2 \mathrm{~mm}$. 

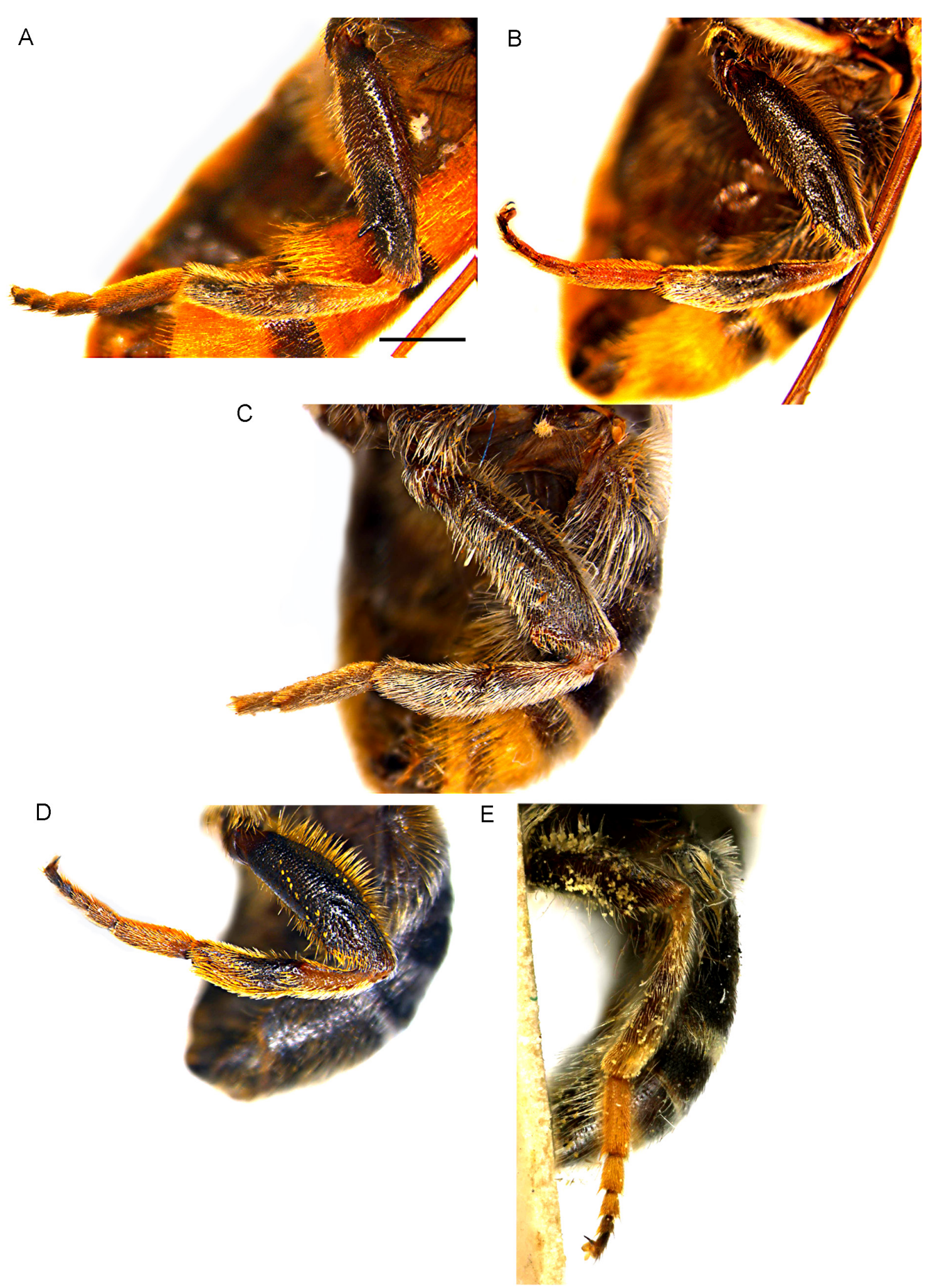

Fig. 16. Female metaleg, lateral view. A. Merodon bombiformis Hull, 1944 (NMSA; FSUNS ID 04278). B. M. multifasciatus Curran, 1939 (FSUNS; FSUNS ID ZA5_243). C. M. zebra Vujić \& Radenković sp. nov., paratype (NHMW; FSUNS ID 05725). D. M. lotus Vujić \& Radenković sp. nov., paratype (NMSA; FSUNS ID ZA6_067). E. M. nasicus Bezzi, 1915, lectotype (BMNH NHMUK010369943). Scale $b a r=2 \mathrm{~mm}$. 


\title{
Period of flight and distribution (Fig. 1)
}

Merodon nasicus is known from Kenya, Ethiopia, Eritrea and Burundi. On the basis of our data, the flight period was found to be in March and from September to October. This species occurs only on high mountains at elevation between 2000 and $2500 \mathrm{~m}$ a.s.l. in vegetation of montane forest-tundra (Sayre et al. 2013).

\author{
Merodon vittatus Vujić \& Likov sp. nov. \\ urn:lsid:zoobank.org:act:591CEDD4-80E4-4978-B13E-3F66511C9026 \\ Figs 1, 4C, 5C, 6C, 7D-F, 8B, 9C, 10C, 11C, 12C, 18C, 21E
}

\section{Diagnosis}

Brown, large (12 mm), striped species, with golden yellow pile on the tip of abdomen (Fig. 5C); frons and postpronotum dark brown; scutum with five pollinose vittae (Fig. 10C); metafemur with distinct apical thorn on triangular process (Figs 6C, 8B); terga 3 and 4 with broad medial pollinose fasciae (Fig. 5C). Similar to Merodon multifasciatus from which differs only in male genitalia with narrow, S-shaped posterior lobe of surstyle, gradually narrowing toward tip (Fig. 7D: pl) (in M. multifasciatus broad, slightly curved, with almost parallel sides and rounded apex (Fig. 13D: pl).

\section{Etymology}

The specific epithet 'vittatus' 'wearing or carrying a vitta', an adjective, refering to the broad golden gray fasciae on the terga $2-4$.

\section{Material examined}

\section{Holotype}

GHANA 1 đ; Transvolta Togoland, Amedzofe; $6.853^{\circ}$ N, $0.433^{\circ}$ E; 2 Nov. 1959; N.D. Jago leg.; FSUNS ID 04085; NBCN.

\section{Description}

Length: large species, body $12 \mathrm{~mm}$, wing $8.5 \mathrm{~mm}(\mathrm{n}=1)$.

\section{Male}

Head (Figs 4C, 9C, 11C, 12C). Antenna (Fig. 4C) reddish-yellow; pedicel elongated, approximately as long as basoflagellomere (relation scape: pedicel : basoflagellomere $=1.0: 2.5: 2.5$ ); basoflagellomere concave dorsally, with acute apex; arista yellow to reddish, thickened basally, 1.7 times as long as basoflagellomere. Face dark brown, covered with gray pollinosity and whitish pile, except on bare medial vitta that occupies $1 / 4$ width of face. Oral margin protruded (Fig. 9C). Frons dark brown, inflated, covered with gray pollinosity and whitish pile. Vertical triangle isosceles (Fig. 12C), reddish, covered with a long, whitish pile. Ocellar triangle equilateral. Eye pile dense, gray, slightly longer than scape. Eye contiguity about 15 ommatidia long. Occiput reddish-brown, pollinose, covered with a whitish pile.

Thorax (Figs 6C, 8B, 10C). Mesonotum black, except lateral side of scutum including postpronotum, postalar callus, and posterior margin of scutellum red-brown; covered with grayish-yellow pile; scutum with five pollinose longitudinal vittae (Fig. 10C). Pleuron black to dark brown, covered with gray pollinosity and the following parts with whitish to yellow pile: anterior part of proepimeron, posterior part of anterior anepisternum, most of the posterior anepisternum except anterior end, antero-ventral and postero-dorsal part of katepisternum and anepimeron. Wing hyaline, with dense microtrichia and yellow to brown veins. Calypter pale yellow. Halter with pale yellow pedicel and capitulum. All three femora dark brown; tibiae mostly reddish, medially dark brown; tarsi reddish (Figs 6C, 8B). Metatrochanter without calcar. Metafemur moderately thickened, with less developed apicoventral triangular lamina, 
more or less dentate, the apical dens is distinct (Figs 6C, 8B). Pile on legs predominantly whitish to yellow.

AbDomen (Fig. 5C). Black to dark brown. Tergum 1 with gray pollinosity, covered with black basal sockets of pile and with short pale yellow pile; tergum 2 with pollinose posterior margin and narrow medial fascia, interrupted in the middle, covered with pale yellow pile; terga 3 and 4 with pollinose posterior margin and broad medial fasciae (Fig. 5C); tergum 3 covered with pale yellow pile except anterior half with few short black pile; tergum 4 covered with long golden-yellow pilosity (Fig. 5C). Sterna reddish-brown, covered with pale yellow pile.

GeNitalia (Figs 7D-F, 18C). Posterior lobe of surstyle S-shaped, gradually narrowing toward tip (Fig. 7D: pl); anterior margin of surstyle angular (Fig. 7D: ams); cercus elongated (Fig. 7D: c). Hypandrium with theca medially distinctly narrowed (Fig. 7F). Lateral sclerite of aedeagus small (Fig. 18C: s).
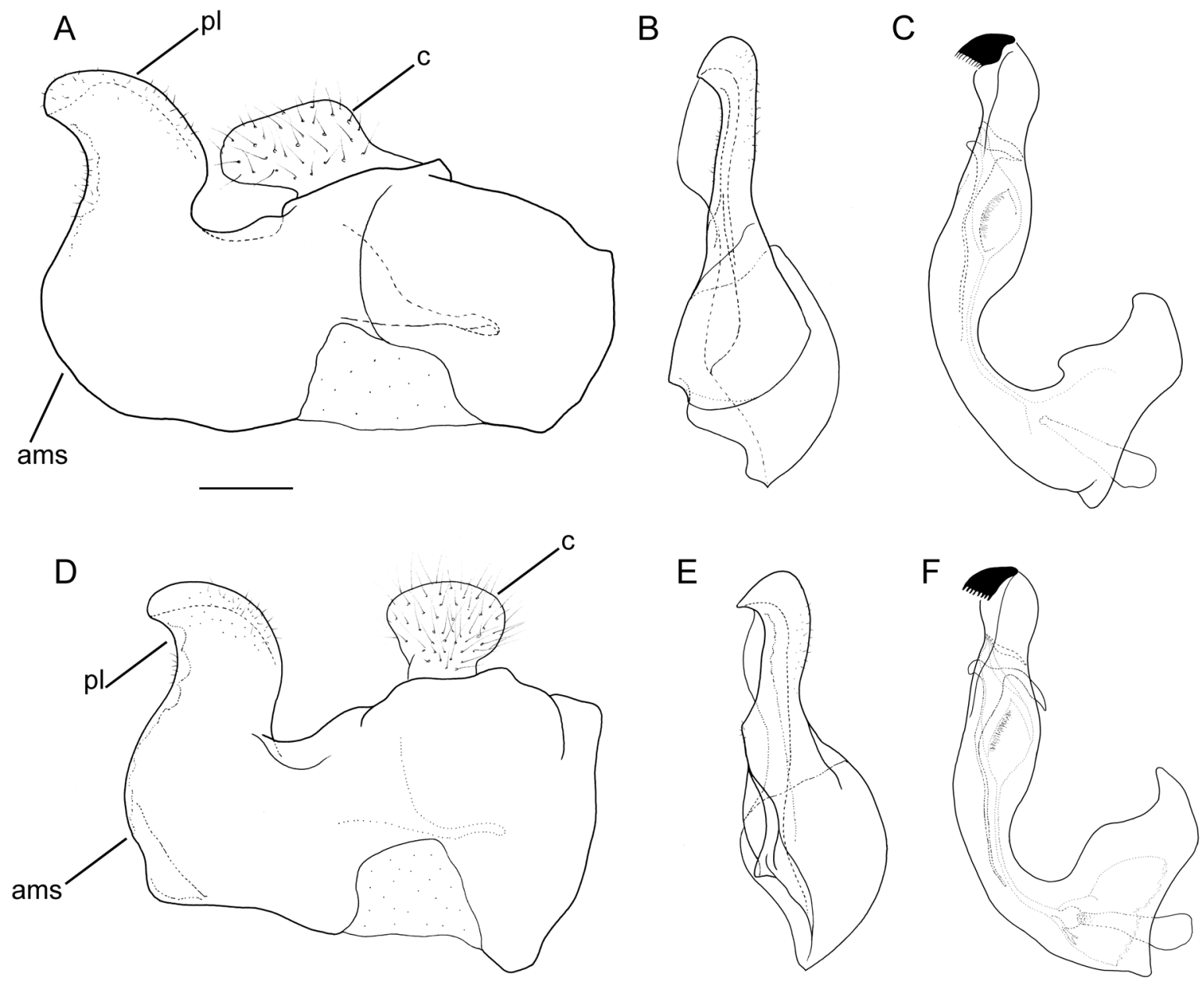

Fig. 17. Merodon lotus Vujić \& Radenković sp. nov., male genitalia. Paratypes. A-C. Specimen FSUNS (FSUNS ID ZA6_066). D-F. Specimen FSUNS (FSUNS ID ZA6_060). A, D. Epandrium, lateral view. B, E. Epandrium, ventral view. C, F. Hypandrium, lateral view. Abbreviations: ams = anterior margin of surstyle; $\mathrm{c}=$ cercus; $\mathrm{pl}=$ posterior lobe of surstyle. Scale bar $=0.25 \mathrm{~mm}$. 


\section{Female \\ Unknown. \\ Period of flight and distribution (Fig. 1)}

Only the holotype is known, which originated from Ghana and was collected in November. It was found in a deciduous forest-woodland savanna type of vegetation.

Merodon zebra Vujić \& Radenković sp. nov. urn:1sid:zoobank.org:act:9B6ABB0D-7FF4-4217-9C23-FBD06F8D80F1

Figs 1, 3D, 4D, 5D, 6D, 7A-C, 9D, 10D, 11D, 12D, 14C, 15C, 16C, 22D-F

\section{Diagnosis}

Large (10-12 mm), dark, striped species, with golden-yellow pile on the tip of abdomen (Fig. 5C). Scutum with conspicuous pollinose vittae (Fig. 10D); metafemur with medium sized apical dens on triangular lamina (Figs 6D, 16D); terga 3 and 4 with broad medial pollinose fasciae (Fig. 5D); male genitalia with S-shaped posterior lobe of surstyle, gradually narrowing toward tip (Fig. 7A: pl); anterior margin of surstyle rounded in lateral view (Fig. 7A: ams). Similar to Merodon vittatus sp. nov. and M. multifasciatus from which differs in less distinct dens on apicoventral triangular lamina of metafemur; shorter eye contiguity, about 10 ommatidia long, while in M. vittatus sp. nov. is about 15 , and in M. multifasciatus about 15-20; and shape of male genitalia (rounded anterior margin of surstyle (Fig. 7A: ams), in M. vittatus sp. nov. angular in lateral view (Fig. 7D: ams); narrow, S-shaped posterior lobe of surstyle, gradually narrowing toward tip (Fig. 7A: pl), in M. multifasciatus broad, slightly curved, with almost parallel and rounded apex (Fig. 13D: pl).

A

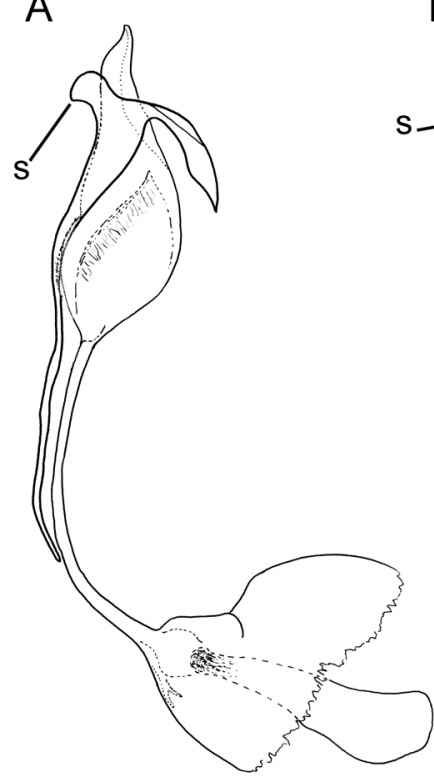

B

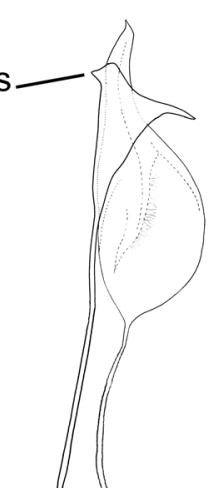

C

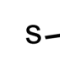

C

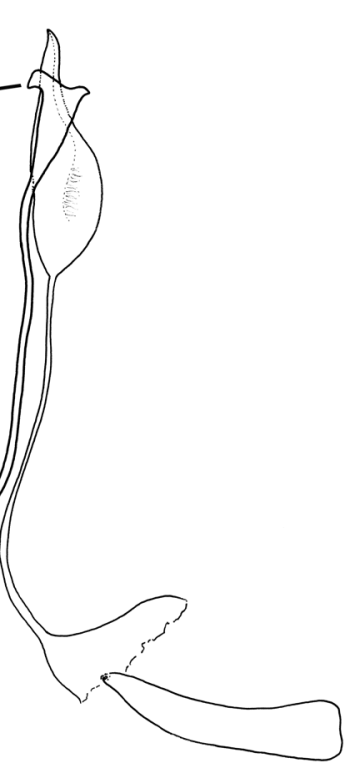

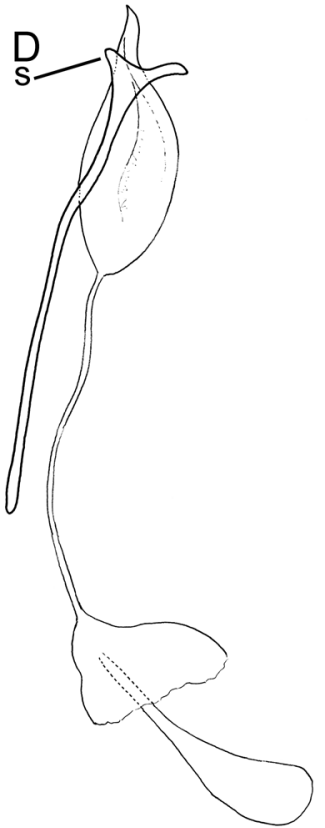

Fig. 18. Male genitalia, aedeagus, lateral view. A. Merodon lotus Vujić \& Radenković sp. nov., paratype (FSUNS; FSUNS ID ZA6_060). B. M. multifasciatus Curran, 1939 (NBCN LML-05-3; FSUNS ID 03909). C. M. vittatus Vujić \& Likov sp. nov., holotype (NBCN; FSUNS ID 04085). D. M. nasicus Bezzi, 1915 (TAUI; FSUNS ID 04972). Abbreviation: s = lateral sclerite of aedeagus. Scale bar $=$ $0.25 \mathrm{~mm}$. 


\section{Etymology}

The specific epithet 'zebra' was given as a reference to the African equine, zebra with a distinctive black and white striped coat, pattern like in this Merodon species. It is to be treated as a noun in apposition. The name 'zebra' was proposed by W. Hurkmans in an unpublished manuscript, but unfortunately the designated holotype belongs to M. multifasciatus. We decided to keep this descriptive name for this African endemic.

\section{Material examined}

\section{Holotype}

DEMOCRATIC REPUBLIC OF THE CONGO • 1 ते; Lubumbashi (former Elisabethville); $11.589^{\circ} \mathrm{S}$, $27.427^{\circ}$ E; 4 Feb. 1923; Dr M. Bequaert leg.; FSUNS ID 25089; RMCA.

\section{Paratypes}

DEMOCRATIC REPUBLIC OF THE CONGO • 1 đ, 1 o ; Lualaba River; $7.809^{\circ} \mathrm{S}, 26.919^{\circ} \mathrm{E}$; 562 m a.s.1.; 2500-4000 ft; 17 May 1907; S.A. Neave leg.; FSUNS ID 03314, 03911; BMNH • 1 đ; same collection data as for preceding; 30 May 1907; FSUNS ID 03912; NBCN AM-05-073 • 1 1 ; ; same collection data as for preceding; FSUNS ID 03316, 03910; BMNH • 1 ¿ ; same collection data as for preceding; 31 May 1907; FSUNS ID 03315; BMNH 1 q; Kasongo; $4.45^{\circ} \mathrm{S}, 26.666^{\circ} \mathrm{E}$, $661 \mathrm{~m}$ a.s.1; 7 Nov. 1912; Dr M. Bequaert leg.; FSUNS ID 25090; RMCA 1 q; same collection data as for holotype; 16 Jan. 1921; FSUNS ID 25091; RMCA • 1 đ̊; Lubumbashi (former Elisabethville), R. Kimilol; $11.589^{\circ}$ S, $27.427^{\circ}$ E; Dec. 1928; Dr M. Bequaert leg.; FSUNS ID 05174; NBCN.

TANZANIA - 1 ; ; Tanganyika Territory (former), Ugano, Matengo Highland WSW of Songea; $10.949^{\circ} \mathrm{S}, 34.934^{\circ} \mathrm{E}$; 1500-1700 m a.s.1.; 11-20 Jan. 1936; H. Zerny leg.; FSUNS ID 05725; NHMW.

\section{Description}

Length: large species, body $10-12 \mathrm{~mm}$, wing $7-8 \mathrm{~mm}(\mathrm{n}=4)$.

\section{Male}

HeAD (Figs 4D, 9D, 11D, 12D). Antenna (Fig. 4D) reddish-brown; pedicel elongated, approximately as long as basoflagellomere (relation scape : pedicel : basoflagellomere $=1.0: 2.5: 2.5$ ); basoflagellomere concave dorsally, with acute apex; arista light brown to dark brown, thickened basally, 1.6 times as long as basoflagellomere. Face from black to dark brown, covered with gray pollinosity and whitish pile, except on bare medial vitta that occupies $1 / 4$ width of face. Oral margin protruded (Fig. 9D). Frons from black to reddish, inflated, covered with gray pollinosity and whitish pile. Vertical triangle isosceles (Fig. 12D), usually dark brown, predominantly covered with a long, yellow pile. Ocellar triangle equilateral. Eye pile dense, gray, slightly longer than scape. Eye contiguity about 10 ommatidia long. Occiput reddish, pollinose, covered with yellow pile.

Thorax (Figs 6D, 10D). Mesonotum black, except postpronotum and posterior margin of scutellum brown-reddish, covered with reddish-yellow pile; scutum with five pollinose longitudinal vittae (as on Fig. 10D). Pleuron black to dark brown, covered with gray pollinosity and the following parts with whitish to yellow pile: anterior part of proepimeron, posterior part of anterior anepisternum, most of the posterior anepisternum except anterior end, antero-ventral and postero-dorsal part of katepisternum, anepimeron and metasternum. Wing hyaline, with dense microtrichia and yellow to brown veins. Calypter pale yellow. Halter with yellow pedicel and capitulum. All three femora dark brown to black; tibiae mostly reddish, medially black to dark brown; tarsi reddish, except darker apical two tarsomeres (Fig. 6D). Metatrochanter without calcar. Metafemur moderately thickened, with less developed apicoventral triangular lamina, more or less dentate, the apical dens is distinct (Fig. 6D). Pile on legs predominantly whitish to yellow. 
ABDomen (Fig. 5D). Black to dark brown. Tergum 1 black, usually with pollinosity, covered with short whitish to yellow pile; tergum 2 with pollinose posterior margin and narrow medial fascia, in some specimens interrupted in the middle, with whitish pile except posterior half between pollinose fasciae covered with short black pilosity; terga 3 and 4 with pollinose posterior margin and broad medial fasciae; anterior half of tergum 3 covered with short black pile; tergum 4 covered with long golden yellow pilosity. Sterna reddish-brown, covered with white or yellow pile.

Genitalia (Figs 3D, 7A-C). Posterior lobe of surstyle narrow, bent, S-shaped, gradually narrowing toward tip (Fig. 7A: pl); anterior lobe of surstyle undeveloped (Fig. 7A); anterior margin of surstyle rounded in lateral view (Fig. 7A: ams); cercus square like (Fig. 7A: c). Hypandrium with theca medially distinctly narrowed (Fig. 7C: marked with arrow). Lateral sclerite of aedeagus small (Fig. 3D: s).

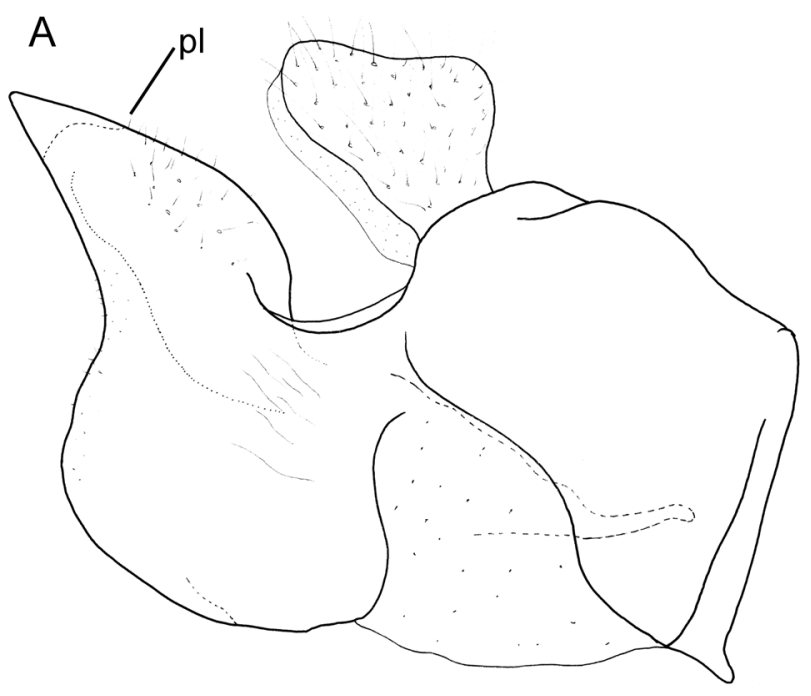

B
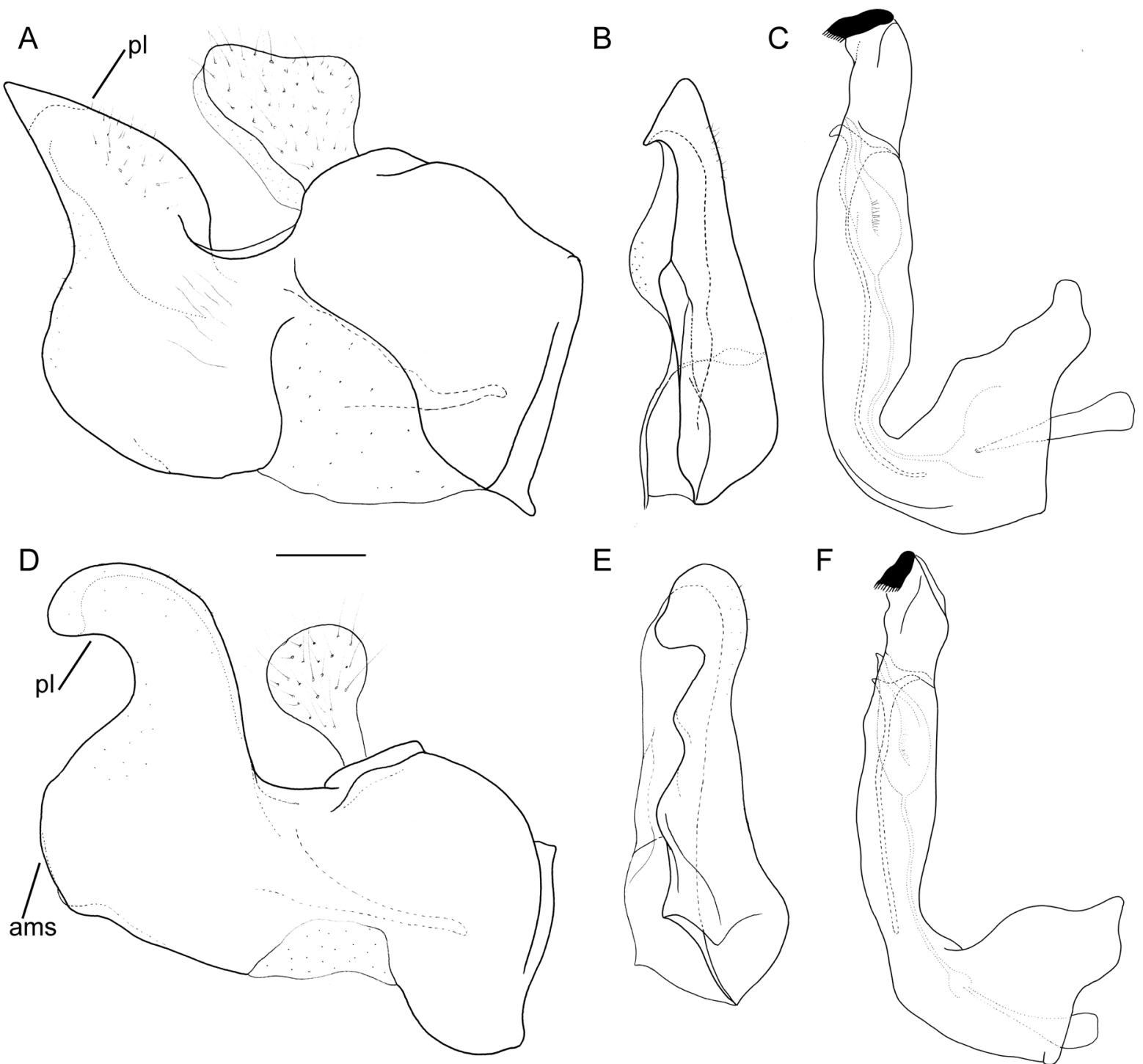

E

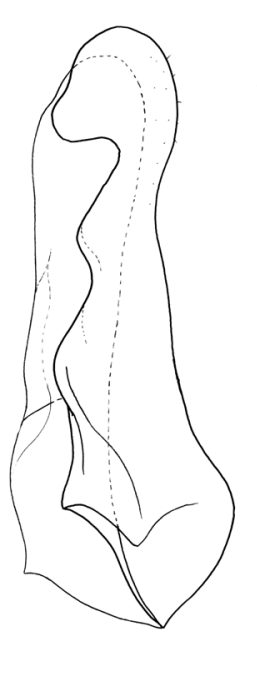

$\mathrm{F}$

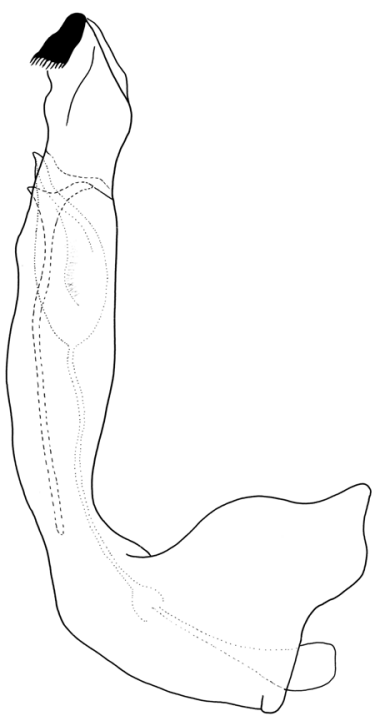

Fig. 19. Male genitalia. A-C. Merodon funestus (Fabricius, 1794) (FSUNS; FSUNS ID F99). D-F. M. nasicus Bezzi, 1915 (TAUI; FSUNS ID 04792). A, D. Epandrium, lateral view. B, E. Epandrium, ventral view. C, F. Hypandrium, lateral view. Abbreviations: ams = anterior margin of surstyle; $\mathrm{pl}=$ posterior lobe of surstyle. Scale bar $=0.25 \mathrm{~mm}$. 
Female (Figs 14C, 15C, 16C)

Similar to the male except for normal sexual dimorphism: metafemur usually with small apical dens on triangular lamina (Fig. 16C); frons with non pollinose medial vitta (Fig. 15C); vertex shiny, non pollinose, mostly covered with black pile.

\section{Period of flight and distribution (Fig. 1)}

Merodon zebra sp. nov. occurs in central parts of Africa (Democratic Republic of the Congo and Tanzania); the flight period may extend over the whole year and probably depends on local circumstances. It appears in a deciduous forest-woodland savanna type of vegetation (Sayre et al. 2013).

\section{Names of Merodon in the Afrotropical fauna with unresolved status}

\section{Merodon edentulus Macquart, 1855 nom. dub., stat. rev.}

Merodon edentulus Macquart, 1855: 90.

This name was introduced by Macquart (1855) based on a single specimen from South Africa. The type material is lost. Curran (1939) stated that this can scarcely be a Merodon because it is said to

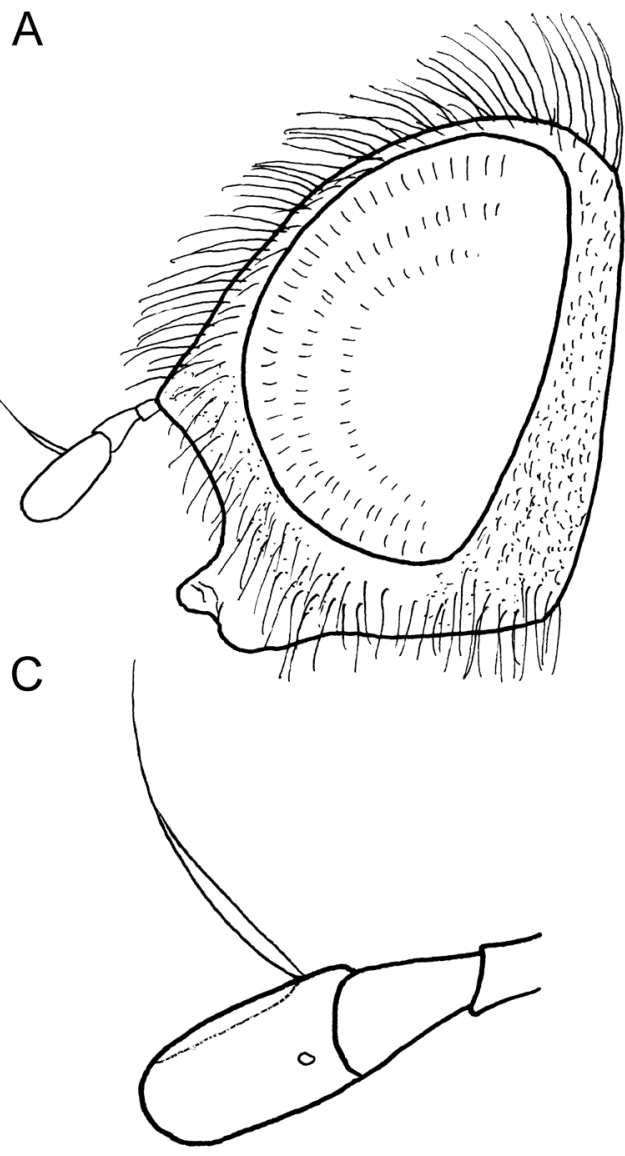

B

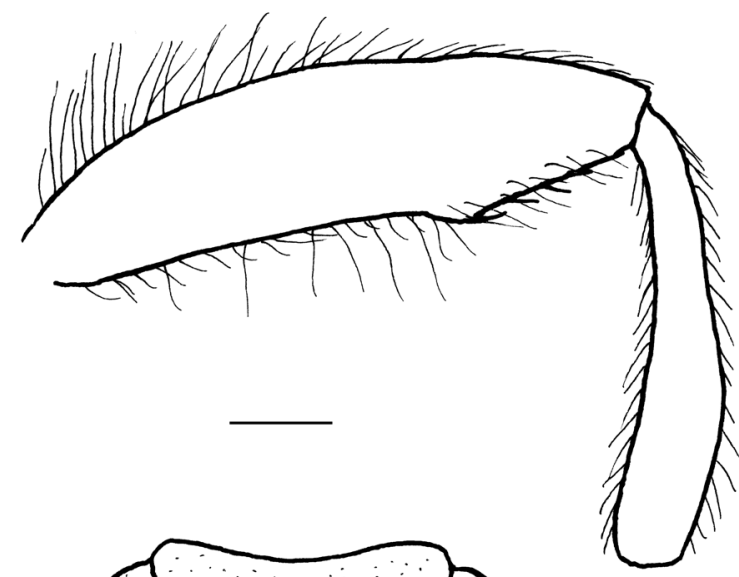

D

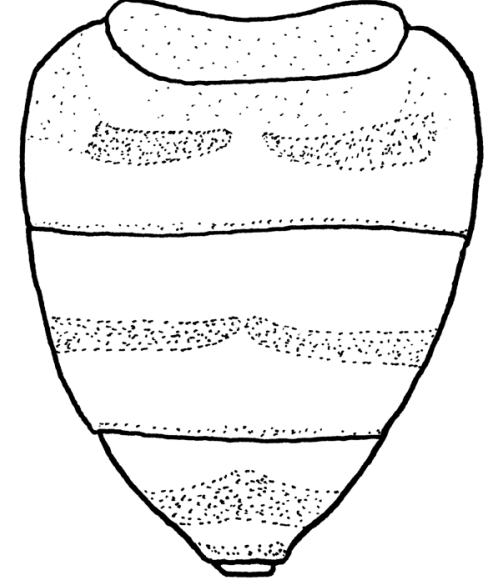

Fig. 20. Merodon nasicus Bezzi, 1915, lectotype, $q$ (BMNH NHMUK010369943). A. Head, lateral view. B. Metafemur and metatibia, lateral view. C. Antenna, lateral view. D. Abdomen, dorsal view. Scale bar $=1 \mathrm{~mm}$. 
A

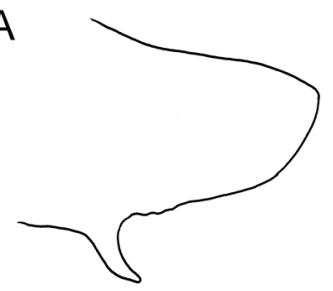

$\mathrm{E}$

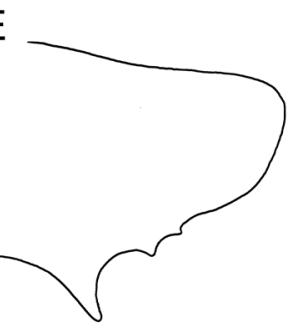

B

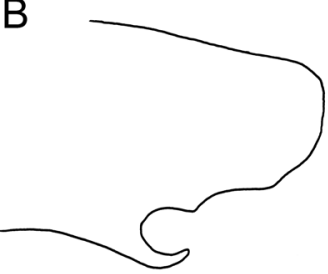

$\mathrm{F}$

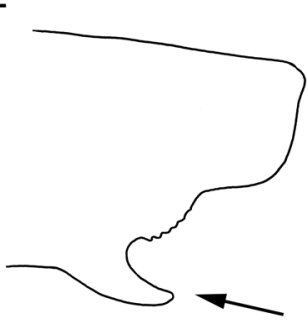

C

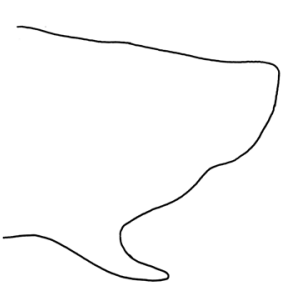

G

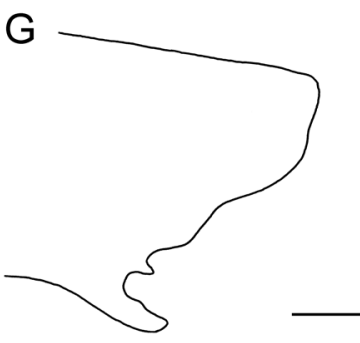

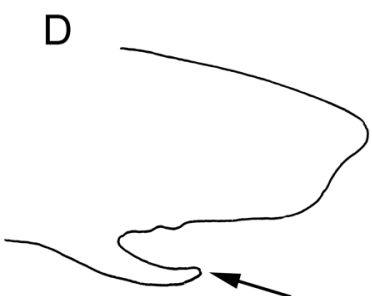

$\mathrm{H}$

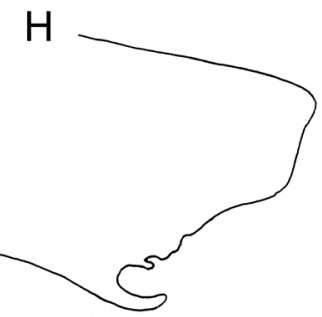

Fig. 21. Apical part of male metafemur, lateral view. A-D. Merodon bombiformis Hull, 1944 A. Specimen FSUNS (FSUNS ID ZA2_079). B. Specimen NMSA (FSUNS ID 04276). C. Specimen NMSA (FSUNS ID 04277). D. Specimen NBCN LML-05-5 (FSUNS ID 04282). E. M. vittatus Vujić \& Likov sp. nov., holotype (NBCN; FSUNS ID 04085). F-G. M. multifasciatus Curran, 1939. F. Specimen NMSA (FSUNS ID 04284). G. Specimen NBCN LML-05-3 (FSUNS ID 03909). H. M. apimina Hull, 1944, paratype (NBCN; FSUNS ID 04086). D, F. Distinct apical dent on triangular process marked with arrow. Scale bar $=1 \mathrm{~mm}$.

A

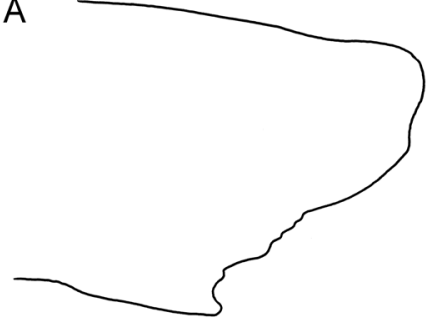

D

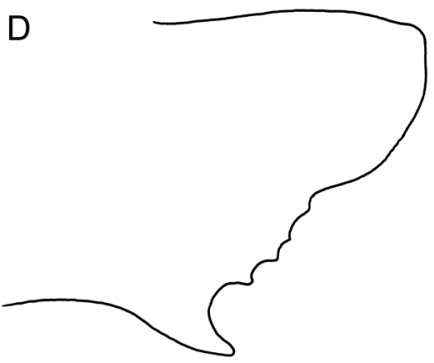

B

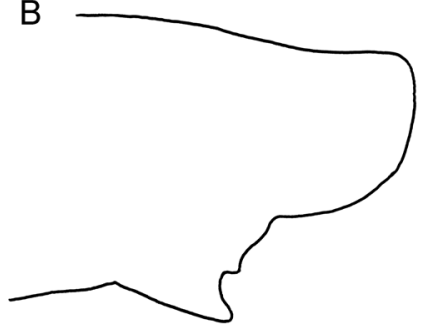

E

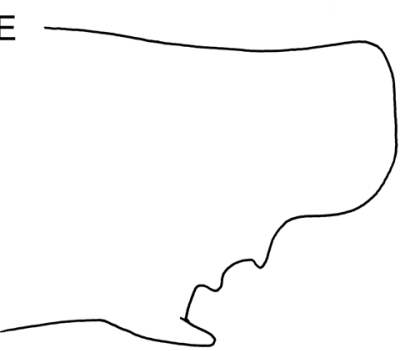

C

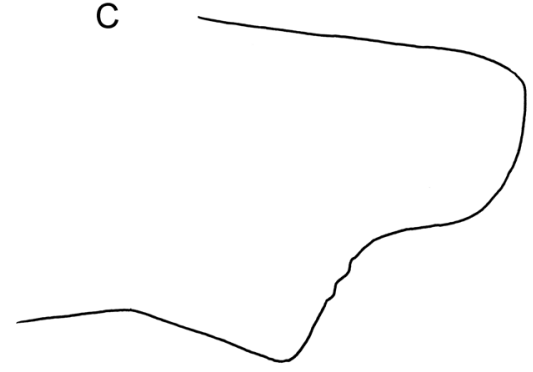

$\mathrm{F}$

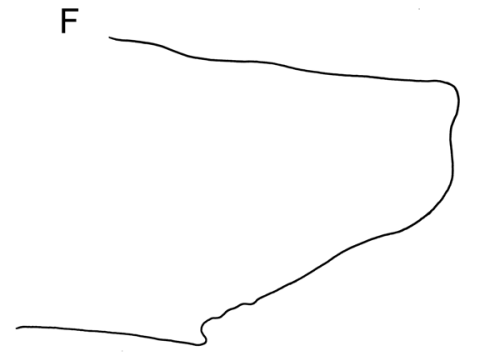

Fig. 22. Apical part of male metafemur, lateral view. A-C. Merodon lotus Vujić \& Radenković sp. nov. Paratypes. A. Specimen FSUNS (FSUNS ID ZA6_052). B. Specimen FSUNS (FSUNS ID ZA6_060). Specimen FSUNS (FSUNS ID ZA6_066). D-F. M. zebra Vujić \& Radenković sp. nov. D. Holotype (RMCA; FSUNS ID 25089). E. Paratype (NBCN; FSUNS ID 05174). F. Paratype (RMCA; FSUNS ID 25090). Scale bar $=1 \mathrm{~mm}$. 
have no thorn (lamina) on the metafemur. Type locality is Cape of Good Hope (RSA) from where no specimen of Merodon was collected. The description is insufficient, even for an identification to the genus level. In the same publication, Macquart (1855) described one other exotic Merodon from South America, Merodon angustiventris Macquart, 1855 which was re-identified as a member of the genus Quichuana Knab, 1913 (Thompson 1988). Therefore, we regard the name Merodon edentulus to be a nomen dubium.

\section{Variability of species in the Merodon bombiformis group}

A variability in shape of the triangular lamina of the metafemur is present in most of the species of the Merodon bombiformis group, as seen in figures of M. bombiformis (Fig. 21A-D), M. lotus sp. nov. (Fig. 22A-C) M. multifasciatus (Fig. 21F-H), M. nasicus (Fig. 23A-B) and M. zebra sp. nov. (Fig. 22DF). The triangular lamina is more or less dentate, and the apical dens is distinct (M. bombiformis, M. multifasciatus, $M$. vittatus sp. nov.) or less distinct, as in M. lotus sp. nov. and M. nasicus. A scutum with conspicuous pollinose vittae, and terga with pollinose posterior margin and medial fasciae, are characters present in most species, except $M$. lotus sp. nov., characterized by the absence of pollinosity on thorax and abdomen (in females a pair of indistinct pollinose fasciae on tergum 4). Width and shape of vittae and fasciae on scutum and terga vary, especially in M. multifasciatus, M. nasicus and M. zebra sp. nov. The colour of the postpronotum, lateral sides of scutum, frons and face in M. multifasciatus vary from reddish-brown to black. Specimens from the south of the range (RSA) are darker than individuals from northern parts (Central Africa).

A

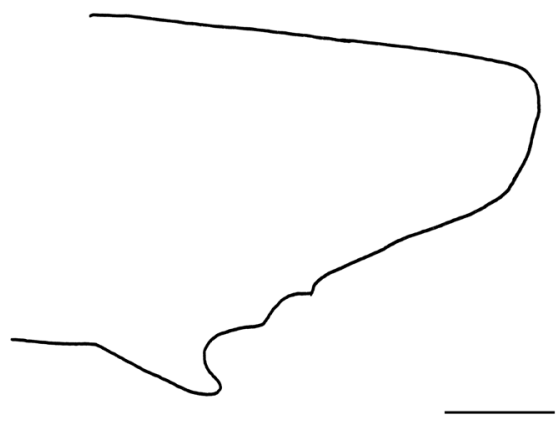

B

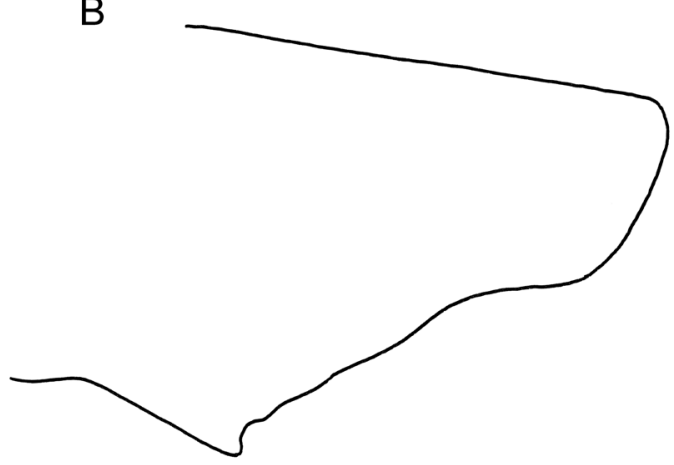

C

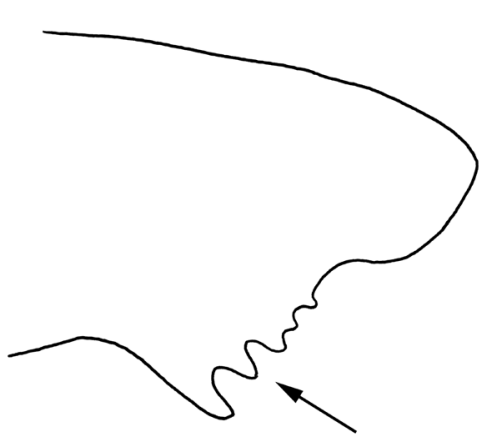

Fig. 23. Apical part of male metafemur, lateral view. A-B. Merodon nasicus Bezzi, 1915. A. Specimen RBINS (FSUNS ID 29176). B. Specimen TAUI (FSUNS ID 04972). C. M. funestus (Fabricius, 1794) (FSUNS; FSUNS ID F98), dentate apicoventral triangular lamina on metafemur marked with arrow. Scale bar $=1 \mathrm{~mm}$. 
VUJIĆ A. et al., Revision of the Merodon bombiformis species group

\section{Key to the species of the Merodon bombiformis species group}

Female of $M$. vittatus sp. nov. is unknown.

1. Species with reddish-yellow/golden face, frons (Figs 9A, 15A), lateral sides of scutum (Figs 10A, 14A) and most of terga (Figs 5A, 14A); oral margin reduced (Fig. 9A); antennal segments very short (Fig. 4A)

Merodon bombiformis Hull, 1944

- Species with mostly dark face, frons, lateral sides of scutum and most of terga; oral margin protruded (Fig. 9B-F); antennal segments longer (Fig. 4B-F)

2. Large, dark/brownish species (body length $10-14 \mathrm{~mm}$ ) with distinct pollinose fasciae on terga 2-4 covered with whitish to yellow pile (Figs 5B-D, 14B-C); tergum 4 with broad medial pollinose fasciae; scutum with five longitudinal pollinose vittae; face dark, mostly covered with gray pollinosity

- Smaller, black species (body length 6-9 $\mathrm{mm}$ ) without or with less distinct pollinose fasciae on terga 2-4 (Fig. 5E-F); tergum 4 with less distinct medial pollinose fasciae; scutum without or with indistinct pollinose vittae; face shiny black without pollinosity

3. Metafemur with shorter and less dense pilosity, especially ventro-laterally (Figs 6B-C, 16B); eye contiguity longer, more than 15 ommatidia long; anterior margin of surstyle angular in lateral view (Figs 7D, 13D)

- Metafemur with long and dense pilosity, especially ventro-laterally (Figs 6D, 16C); eye contiguity shorter, about 10 ommatidia long; anterior margin of surstyle rounded in lateral view (Fig. 7A: ams)

Merodon zebra Vujić \& Radenković sp. nov.

4. Male genitalia with narrow, S-shaped posterior lobe of surstyle, gradually narrowing toward tip (Fig. 7D) (female unknown) Merodon vittatus Vujić \& Likov sp. nov.

- Male genitalia with broad posterior lobe of surstyle, slightly curved, with almost parallel sides and rounded apex (Fig. 13D) Merodon multifasciatus Curran, 1939

5. Terga 2-4 without pollinosity, only with medial fasciae of whitish pile (Figs 5E, 14D) (in female a pair of indistinct pollinose fasciae on tergum 4) .......... Merodon lotus Vujić \& Radenković sp. nov.

- Terga 2-4 with pairs of medial pollinose fasciae, covered with whitish to yellowish pile (Figs 5F, $14 \mathrm{E})$

Merodon nasicus Bezzi, 1915

\section{Collecting effort}

In the period 2011-2018, eight expeditions to RSA were organized with 235 field working days. During these days, 187 specimens of Merodon were collected, among them 11 specimens belonging to the M. bombiformis group, identified as three species. A single specimen of $M$. bombiformis was collected on field day 108 (in 2016) on the Drakensberg Mountains (Fig. 24). Subsequently, we focused on two other taxa of the M. bombiformis group, previously detected in RSA. Namely, Merodon multifasciatus was recorded on day 215 (in 2018) in the province Mpumalanga, near Emgwenya (Waterval Boven) (Fig. 25). A third species was known from a single specimen deposited in the Natural History Museum, London (previously unpublished), with Ceres as locality on the label. We revisited the Ceres area 18 times in the period 2015-2018 and found a population of M. lotus sp. nov. on day 230 (in 2018) of our fieldwork (Fig. 26).

\section{Molecular analysis}

The specimens of the three collected Merodon bombiformis group species (M. bombiformis, M. multifasciatus and M. lotus sp. nov.) were used to extract DNA for molecular analysis. COI sequences 

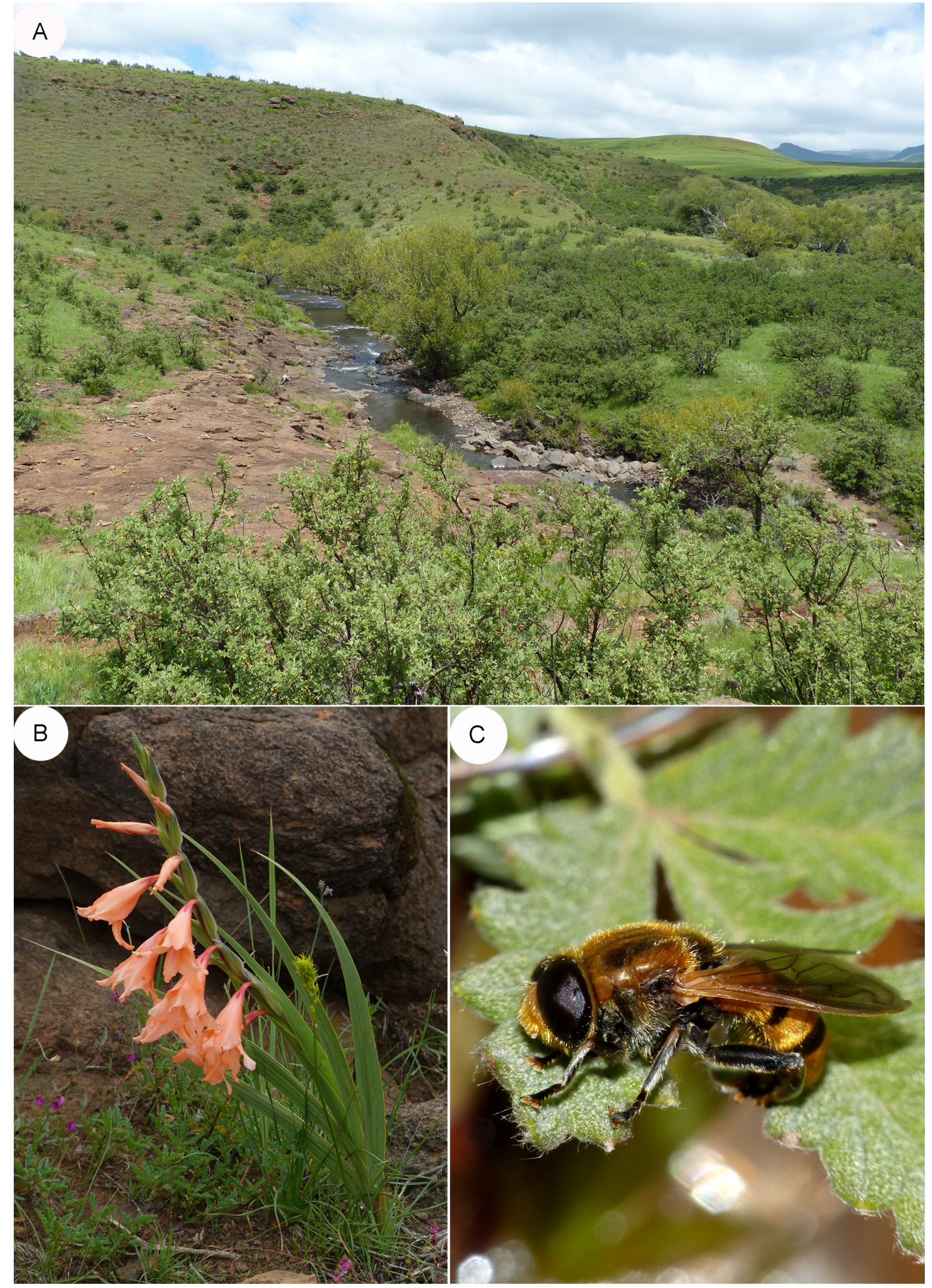

Fig. 24. Habitat of Merodon bombiformis Hull, 1944. A. Drakensberg Mountains, Maclear-Naudes Nek. B. Likely host plant Gladiolus sp. C. Merodon bombiformis male habitus. Photos by Axel Ssymank. 
were produced for one specimen of $M$. bombiformis, two M. multifasciatus and three specimens of M. lotus sp. nov. The obtained COI sequences were 637bp in length and show high uncorrected pairwise genetic distances between species (Table 1). The mean interspecific divergence between species are $\sim 8.4 \%$ between $M$. multifasciatus and $M$. lotus sp. nov., $\sim 14.9 \%$ between $M$. bombiformis and M. multifasciatus, and $\sim 15.5 \%$ between $M$. bombiformis and M. lotus sp. nov.

The ML phylogenetic analysis of the concatenated dataset (1858 bp long) consisting of one mitochondrial gene (COI) and two nuclear ribosomal genes (28S and 18S) produced the tree presented in Fig. 27. Merodon multifasciatus and M. lotus sp. nov. are resolved in a clade with high bootstrap support value
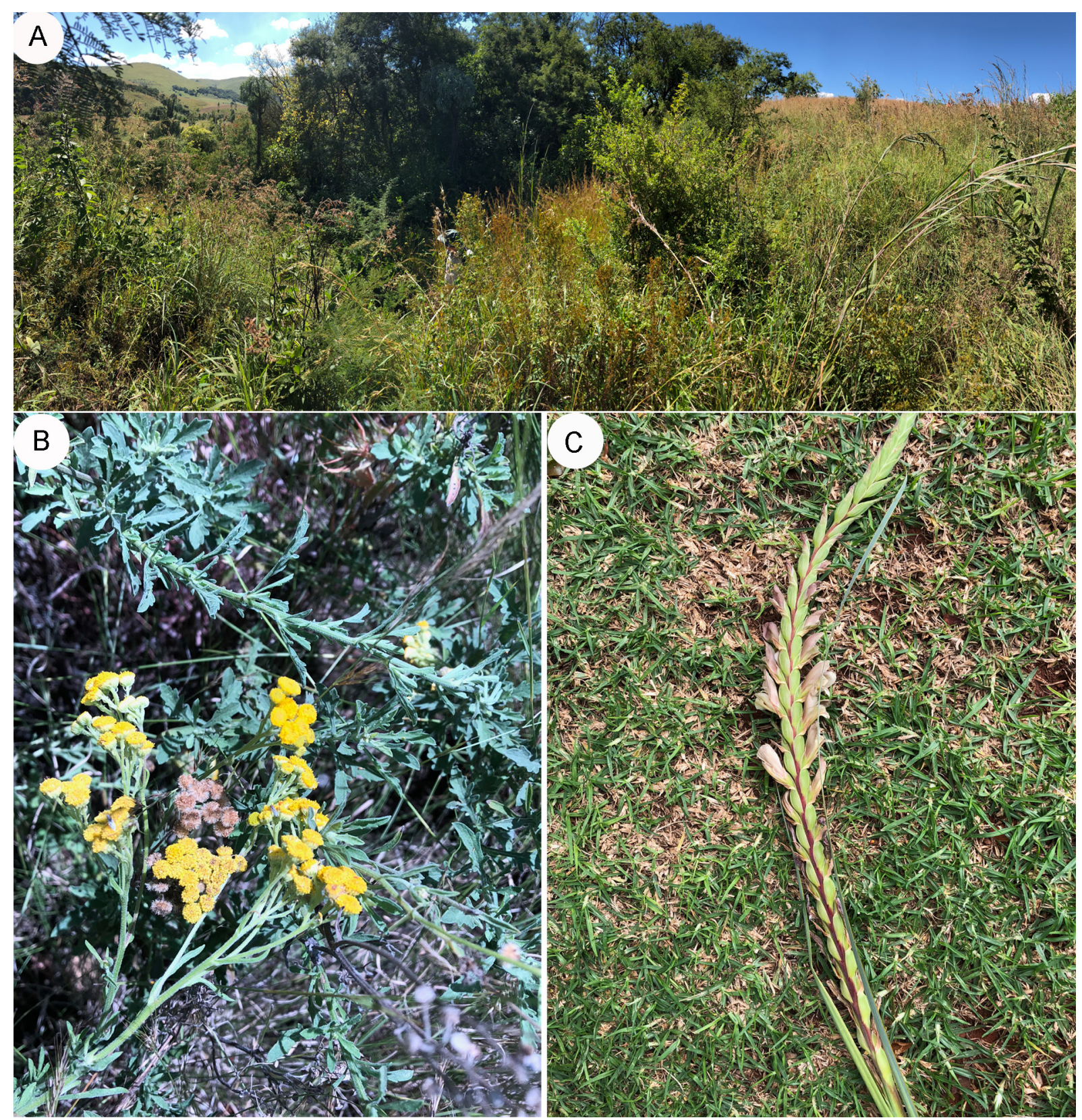

Fig. 25. Habitat of Merodon multifasciatus Curran, 1939. A. Mpumalanga, near Emgwenya (Waterval Boven). B. Nidorella auriculata DC. C. Gladiolus sericeovillosus Hook. Photos by Branka Božičić Lothrop. 
Table 1. Uncorrected pairwise distances of COI gene sequences among specimens of species in the M. bombiformis species group.

\begin{tabular}{llrlrl}
\hline & 1. & 2. & 3. & 4. & 5. \\
\hline 1. M. bombiformis AF68 & & & & & \\
2. M. multifasciatus ZA5_242 & 0.149 & & & & \\
3. M. multifasciatus ZA5_243 & 0.149 & 0.000 & & & \\
4. M. lotus sp. nov. AU1710 & 0.157 & 0.086 & 0.086 & & \\
5. M. lotus sp. nov. AU1712 & 0.154 & 0.082 & 0.082 & 0.005 & \\
6. M. lotus sp. nov. AU1714 & 0.154 & 0.082 & 0.082 & 0.005 & 0.000 \\
\hline
\end{tabular}
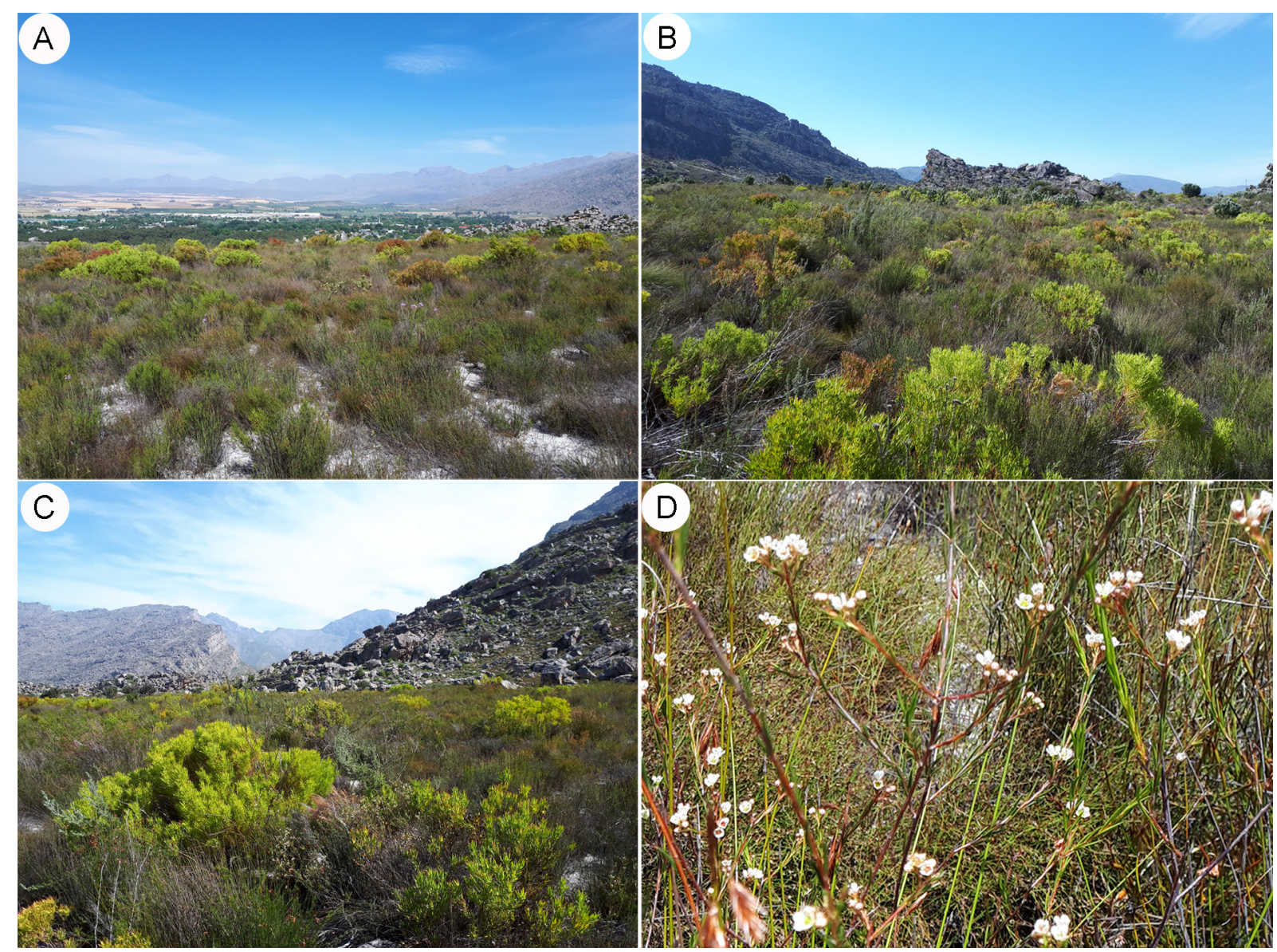

Fig. 26. Habitat of Merodon lotus Vujić \& Radenković sp. nov. A-C. Ceres. D. Coleonema sp. Photos by Ante Vujić. 
(100). Merodon bombiformis represents a separate branch within the aureus lineage, but this placement on the tree has low support (bootstrap value $<50$ ).

\section{Discussion}

The majority of species of Merodon are present in Southern Europe and Asia Minor, which coincides with the high diversity of geophytes (their larval host plants) in these regions. Out of a total of ca 160 species of Merodon, based on recent literature and the present work, only 16 are known from the Afrotropical region. Of the five monophyletic lineages of Merodon (Vujić et al. 2019a), only two, aureus and desuturinus, have representatives in the Afrotropical region, as well as in the Palaearctic. The Merodon desuturinus lineage consists of two clearly separate groups of species based on both adult morphological and molecular data: the Palaearctic group with four species (Vujić et al. 2018) and the Afrotropical group with 10 (Radenković et al. 2018; Djan et al. 2020).

The Merodon bombiformis group consists of six related, though clearly morphologically separated, species distributed in central and southern Africa. The most divergent species are M. bombiformis, with

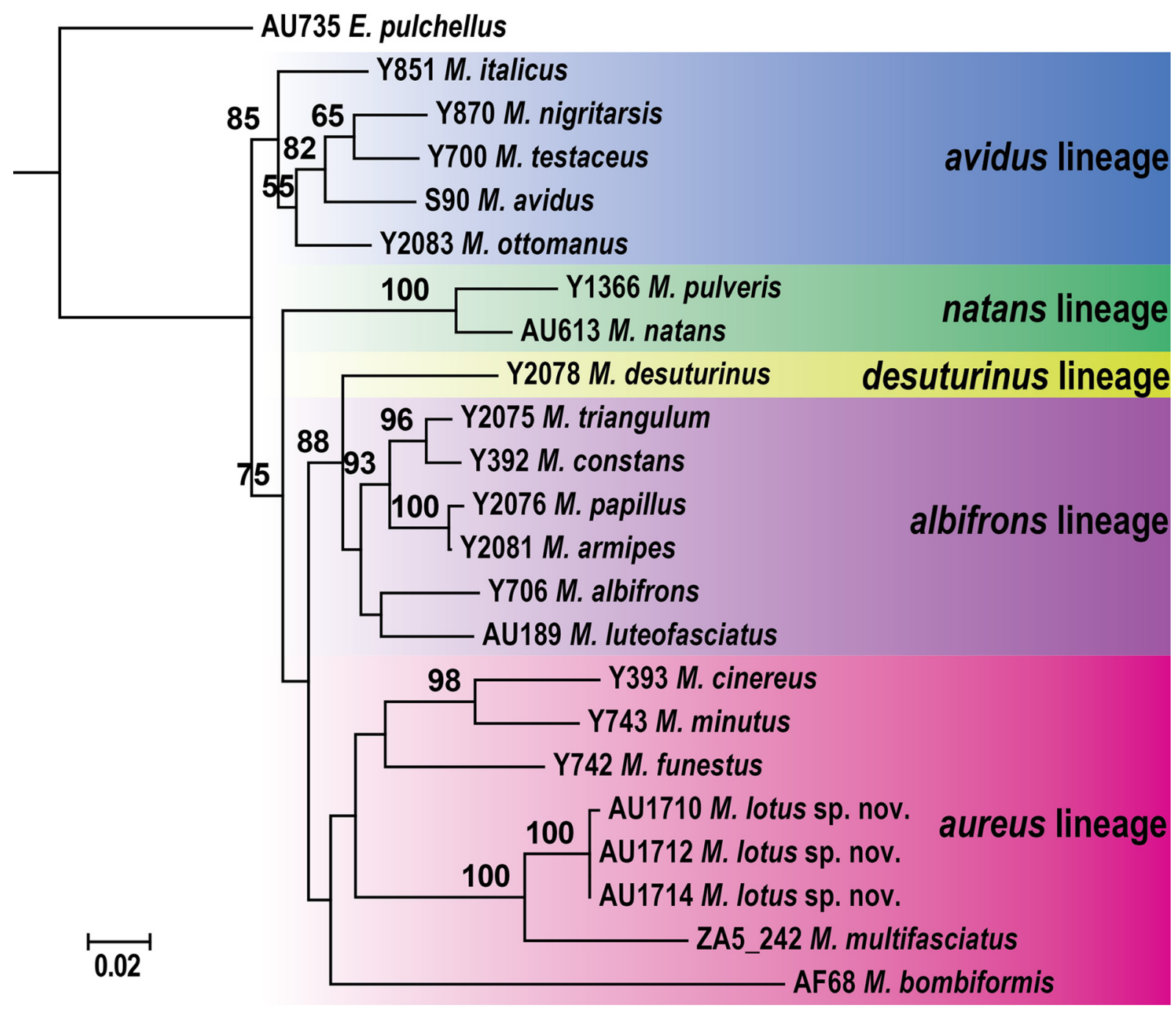

Fig. 27. Maximum likelihood tree from the concatenated data set with COI, $28 \mathrm{~S}$ and $18 \mathrm{~S}$ rRNA gene sequences. 
several distinct characteristics (reddish-yellow face and frons; reduced mouth parts; antennal segments very short) and $M$. lotus sp. nov., with the posterior half of the scutum and the terga lacking pollinosity. Three species, M. multifasciatus, M. vittatus sp. nov. and M. zebra sp. nov., are very similar in external morphology with small differences between their male genitalia and in the size of the distal tooth on the apicoventral triangular lamina of the metafemur, the pilosity of the metafemur, and the length of the eye contiguity. For three species of the M. bombiformis group, M. bombiformis, M. lotus sp. nov. and M. multifasciatus, we have molecular data showing they are clearly genetically separated with a high COI divergence. Merodon bombiformis is morphologically similar to M. multifasciatus, but genetically highly divergent.

The Merodon bombiformis group and M. funestus differ from other species and groups among the aureus lineage in two apomorphic characters, an elongate pedicel and small lateral sclerite of the aedeagus. Lateral sclerite of the aedeagus are absent in other groups and species belonging to the $M$. aureus lineage. Morphologically, the M. bombiformis group can be distinguished from M. funestus by the absence of a projection on the metatrochanter in the males and a less dentate apicoventral triangular lamina on the metafemur. Based on the general external morphology, two species of the M. bombiformis group, Merodon lotus sp. nov. and M. nasicus, show a clear resemblance to M. funestus. Merodon lotus sp. nov. and M. multifasciatus are resolved in one highly supported clade, while M. funestus has an uncertain position on the phylogenetic tree. This is an indication of the monophyly of the M. bombiformis group, supported by morphological data. But, additional species, representatives of different groups of the aureus lineage, require to be included in order to resolve the phylogeny of the whole lineage.

Merodon bombiformis possesses some distinct apomorphic characters, such as a reduced mouthpart, reddish-yellow face and frons, convex frons, pedicel with strong setulae, basoflagellomere short, rounded, with a medial fossette. Reduced mouthparts (oral margin) covered by microtrichia are also present in the M. planifacies subgroup (Vujić et al. 2018; Djan et al. 2020). During 32 fieldwork days in the Drakensberg Mountains, spread over seven years of research conducted in RSA, members of our team collected 51, and observed twice as many, specimens of the M. planifacies subgroup (Djan et al. 2020).

There were no observed flower visits by any of the adult specimens during all of these fieldwork days. Adults do not visit flowers because they do not feed due to an adult life strategy adapted to fasting, and therefore they do not need well-developed mouthparts (Vujić pers. obs.). We can suppose that the same situation exists in M. bombiformis. We collected 11 specimens from the M. bombiformis group during our fieldwork. Two specimens of M. multifasciatus were recorded on flowers of Nidorella auriculata; all of the eight specimens of $M$. lotus sp. nov. were on flowers of Coleonema sp. By contrast, a single specimen of $M$. bombiformis was recorded sitting on the leaves of bushes.

\section{Placement of the Merodon bombiformis group in the Merodon phylogeny}

As stated, previous studies on the phylogeny of Merodon resolve five evolutionary lineages: albifrons, aureus, avidus-nigritarsis, desuturinus, and natans (Vujić et al. 2019a). The results of all of these studies were based on analysis of COI and 28S rRNA gene sequences (Mengual et al. 2006; Vujić et al. 2012; Radenković et al. 2018), while a new study on the phylogeny of Merodon based on a combined analysis of molecular and morphological data is currently under preparation (Veselić in prep). In this paper, we present a preliminary phylogeny of Merodon based on combined COI, 28S rRNA and 18S rRNA gene sequences with the main goal to resolve the M. bombiformis group relationship in the aureus lineage. We confirmed the monophyly of the five Merodon lineages, although the aureus and albifrons lineages both have low bootstrap support $(<50)$, possibly as a result of a limited number of species per lineage included in the analysis. The M. bombiformis group is resolved within the aureus lineage as paraphyletic. However, due to a limited number of specimens included in the analysis, our results must be interpreted 
VUJIĆ A. et al., Revision of the Merodon bombiformis species group

with caution. Thus, more comprehensive analysis which will include additional specimens to resolve the phylogeny of the $M$. bombiformis group with more certainty.

\section{Distribution and diversity}

The Merodon bombiformis group comprises only six species with an extremely low number of collected specimens. Before we started our research, only 42 specimens of the $M$. bombiformis group had been detected in all available collections (26 males and 16 females). The initial aim of our research in the Republic of South Africa (RSA) was to collect fresh specimens of Merodon bombiformis for molecular analysis in order to understand the position of this taxon in the phylogenetic tree of the M. aureus lineage. During eight expeditions, together providing a total of 235 field days (2011-2018), 187 specimens of Merodon were collected, only 11 of them belonging to the M. bombiformis group. The other 176 specimens belong to the $M$. desuturinus lineage, and have partly been reported on in recent publications (Radenković et al. 2018; Djan et al. 2020). We found specimens of all three species of the $M$. bombiformis group known from RSA and these were used in the molecular analysis presented here. The single specimen of M. bombiformis was collected in 2016 after 108 collecting days, on Naudes Nek in the Drakensberg Mountains. We decided to continue research and focus on two other taxa of the $M$. bombiformis group previously detected in RSA, to obtain further molecular data. The other previously described species, M. multifasciatus, was recorded on day 215 in Mpumalanga Province, near Emgwenya (Waterval Boven). The third species was, until our research, undescribed and represented by a single specimen in the BMNH collections. This specimen was collected in 1920, from Ceres. We revisited Ceres area 18 times and at last found a population of the species, described here as M. lotus sp. nov., on day 230 of our fieldwork. Molecular data obtained for M. multifasciatus and M. lotus sp. nov. were resolved in a separate clade within the aureus lineage. The divergence between them supported the proposition that there has been a high genetic divergence among species in the M. bombiformis group, even though our analysis included only 2 and 3 specimens, respectively. We found that it required huge effort to observe, find and collect specimens of Merodon during our fieldwork in RSA. Comparing collection of specimens of Merodon in the Afrotropical region and the Mediterranean, an almost incredible disproportion is observed. The total number of 176 Merodon specimens from 10 species, collected during 235 days of fieldwork in RSA, can be reached in only one day's fieldwork during peak flying season, in species rich Mediterranean localities like Agiassos Mountain on Lesvos Island, Greece (Vujić et al. 2020).

Radenković et al. (2018) discussed the link between Afrotropical and Mediterranean species of Merodon and their host plants. Larvae of Merodon develop in the underground bulbs, corms, or rhizomes of geophytes (Amaryllidaceae, Iridaceae and Hyacinthaceae) or in the surrounding soil, based on the data for species with known immature stages (Hurkmans 1993; Andrić et al. 2014; Ricarte et al. 2017; Preradović et al. 2018). In the Afrotropical region, larvae of the M. planifacies subgroup species have been recorded in bulbs of Merwilla Speta (family Hyacinthaceae) (Radenković et al. 2018) and larvae of M. bombiformis group species in corms of Gladiolus (Iridaceae). The family Hyacinthaceae comprises between 700 and 900 species, mainly distributed in Africa, Europe and SW Asia (Martínez-Azorín et al. 2011 ) and in the family Iridaceae Juss. there are more than 2200 species worldwide (Christenhusz \& Byng 2016). The subfamily Crocoideae Burnett, to which Gladiolus belongs, comprises about 995 species, with most of the species in Africa, Madagascar and Eurasia, but with the highest diversity in South Africa (Goldblatt et al. 2006). These distributional patterns suggest that diversification of these taxa began when North America was already clearly separated from Eurasia. The absence of the genus Merodon on the American continent can thus be linked to historical events in the Hyacinthaceae and Iridaceae. However, the greatest diversity of Hyacinthaceae and Crocoideae is found in South Africa (Pfosser \& Speta 2004; Goldblatt et al. 2006), which is not in congruence with the exceptionally high diversity of Merodon in the Eastern Mediterranean (more than 100 species) as opposed to South Africa (11 species) (Radenković et al. 2018; Djan et al. 2020). Migration of bulb species from southern Africa 
to northern Africa and Eurasia was possible during the late Early Miocene, giving rise to free exchange of flora and fauna between Africa and Eurasia (Rögl 1998; Pfosser \& Speta 2004). Radenković et al. (2018) postulated that diversification in the M. desuturinus group most probably happened much later, during fundamental shifts in the African climate, likewise the diversification in the M. bombiformis group. Generally, the genus Merodon prefers warm, dry, open habitats with numerous geophytes. Radenković et al. (2018) supposed that during the Pliocene-Pleistocene epoch, favorable conditions for species of Merodon (increased aridity and open grasslands) in Africa (De Menocal 2004) most probably allowed faunal transitions. In the case of the M. desuturinus and M. bombiformis groups, this occurred in the opposite direction to the northward trajectory of the Hyacinthaceae; from the eastern Mediterranean (including SW Asia), one lineage migrated to South Africa and another to the western Palaearctic.

Except for Merodon vittatus sp. nov., reported from Ghana in Western Africa, all other species of the M. bombiformis group are known from the southern or eastern parts of Africa. Merodon vittatus sp. nov. occurs in a deciduous forest-woodland savanna. Merodon nasicus is recorded in Eritrea, Ethiopia, Burundi and Kenya, at high altitudes (2000-2500 m a.s.1.). All of these records originate from localities belonging to the Eastern Afromontane Biodiversity Hotspot, which includes several widely scattered, but biogeographically similar mountain ranges in Eastern Africa (Critical Ecosystem Partnership Fund 1996), with vegetation of montane forest-tundra (Sayre et al. 2013). This chain of localities in East Africa supporting representatives of the $M$. bombiformis group continues toward the south with three species which alternate and partly overlap in their ranges. Merodon zebra sp. nov. is recorded from Congo and Tanzania at moderate elevations (500-1700 m a.s.1.), in deciduous forest-woodland savanna. Merodon multifasciatus occurs in RSA, Malawi and Congo, at the same range of elevations as M. zebra sp. nov. (500-1800 $\mathrm{m}$ a.s.1.), in both deciduous forest-woodland savanna and brush-grass savanna. The range of M. zebra sp. nov. and M. multifasciatus overlap in Congo. Merodon bombiformis occurs in RSA and Lesotho over a range of moderate to high altitudes (800-2500 m a.s.1.), in different types of vegetation, including brush-grass savanna, temperate and mountain grasslands and montane foresttundra (Sayre et al. 2013). Merodon bombiformis and M. multifasciatus have a common range in brushgrass savanna in the north of RSA. The localised endemic M. lotus sp. nov. appears in Mediterranean evergreen forest-sclerophyllous shrub in the extreme south of Africa.

All habitats in RSA where we collected specimens from the Merodon bombiformis group are either outside protected areas, or in protected areas with high pressure of human impact, or at sites both unprotected and heavily impacted by human activity. Merodon bombiformis was found on a small patch of natural habitat ( $0.7 \mathrm{ha}$ ) on the slopes of the Drakensberg Mountains far from protected areas, on the east and north of the mountain range. Merodon multifasciatus was recorded on the edge of the city (Emgwenya (Waterwal Boven)) with high human impact and a very small remaining population of the assumed host plant. This situation is similar to that of the habitat of $M$. lotus sp. nov. (0.5 ha), which belongs to Ceres Bergfynbos Reserve (BIOPAMA 2011), but subject to everyday human influence, being on the edge of city infrastructure. Despite our extremely intense collecting efforts in more than 50 localities in the RSA, most of them in protected areas, all specimens found were outside well-preserved National Parks and Reserves. Conservation efforts to protect these small populations of the extremely rare species of the $M$. bombiformis group thus need to be concentrated on the remaining small patches of natural habitats supporting their host plants. Specimens of the genus Gladiolus are clearly visible during the flowering season and very often collected by the local communities.

\section{Host plants}

The data gathered from both the literature (Stuckenberg 1956) and our collecting experiences point to Gladiolus L. (Iridaceae) as the larval host plant for species of Merodon of the bombiformis group (see under larval biology in M. bombiformis and M. multifasciatus). Our field observations from Emgwenya (Waterval Boven), where we collected adults of M. multifasciatus within a habitat containing a natural 
population of Gladiolus sericeovillosus suggests this plant species is the primary natural host plant. Data about distribution of two subspecies of G. sericeovillosus in South Africa show a broad correspondence with the distribution of these two species of Merodon. Gladiolus sericeovillosus subsp. sericeovillosus, the southern subspecies, is especially common in KwaZulu-Natal (Goldblatt \& Manning 1998), from where most known specimens M. bombiformis originated. The range of this Gladiolus extends from Baziya in the northern Eastern Cape to southern Mpumalanga, along a line extending from Ermelo to Barberton (Goldblatt \& Manning 1998). The second subspecies G. sericeovillosus subsp. calvatus (Baker) Goldblatt, extends within the RSA from Mpumalanga through Northern Province into Zimbabwe, where it occurs widely across the eastern half of the country (Goldblatt \& Manning 1998). This subspecies was also detected in Emgwenya (Waterval Boven), where we collected M. multifasciatus. If we conclude from the distribution data that M. bombiformis is connected with G. sericeovillosus subsp. sericeovillosus and M. multifasciatus with G. sericeovillosus subsp. calvatus, then we can predict the occurrence of the latter plant subspecies in Malawi and Congo. A second inference can be drawn from the line from Ermelo to Barberto, which divides these two subspecies. Along this line there are three records of M. bombiformis (Pretoria, Pietermaritzburg and Waterval) and three of M. multifasciatus (Barberton, Nylstroom and Emgwenya (Waterval Boven)). It would be interesting if botanists were to check the taxonomic status of these subspecies: G. sericeovillosus subsp. sericeovillosus and G. sericeovillosus subsp. calvatus. The entomological data provide reasons to believe that they are distinct species-level taxa.

There are a lot of endemic species of the genus Gladiolus in the Ceres area, from where Merodon lotus sp. nov. was described. Examples are G. carneus de la Roche, G. ceresianus Bolus, G. cylindraceus Lewis, all of them Western Cape endemics (Biodiversity Explorer 2000). Further research is needed to determine more precisely the host plant(s) of this localised endemic syrphid species.

\section{Acknowledgements}

We thank the curators of the museums listed in the Materials and methods for facilitating visits and loans for the study of specimens in their care. We are also indebted to Martin Speight, native English speaker for the linguistic revision, valuable comments and editing of the manuscript. We are sincerely grateful to Dr Nigel Wyatt (NHM) for the photos of the type specimen of Merodon nasicus. We wish to thank Neil Crouch and Troos Van Der Merwe for their help with the identification of plant material. The study was supported by the European Commission, Horizon 2020 Marie Sklodowska-Curie action, Research and Innovation Staff Exchange (RISE) Programme: FlyHigh-Insect-plant relationships: insight into biodiversity and new applications (Grant No. 645636), the Ministry of Education, Science and Technological Development, Republic of Serbia, Grant No. 173002, III43002, 451-03-9/202114/200358 and 451-03-9/2021-14/200125, the Provincial Secretariat for Science and Technological Development of the Republic of Serbia (Grant No. 114-451-1125/2014-03 and 114-451-1702/201403) and European Commission, Horizon 2020 project 'ANTARES' (Grant No. 739570).

\section{References}

Andrić A., Šikoparija B., Obreht D., Đan M., Preradović J., Radenković S., Pérez-Bañón C. \& Vujić A. 2014. DNA barcoding applied: identification of the larva of Merodon avidus (Diptera: Syrphidae). Acta Entomologica Musei Nationalis Pragae 54: 741-757.

Belshaw R., Lopez-Vaamonde C., Degerli N. \& Quicke D.L. 2001. Paraphyletic taxa and taxonomic chaining: evaluating the classification of braconine wasps (Hymenoptera: Braconidae) using 28S D2-3 rDNA sequences and morphological characters. Biological Journal of the Linnean Society 73: 411-424. https://doi.org/10.1111/j.1095-8312.2001.tb01370.x 
Bezzi M. 1915. The Syrphidae of the Ethiopian Region based on Material in the Collection of the British Museum (Natural History) with Descriptions of new Genera and Species. British Museum (Natural History), London. https://doi.org/10.5962/bhl.title.10351

Bezzi M. 1921. Syrphidae aethiopicae Musei nationalis hungarici [concl.]. Brotéria: Série Zoológica 19: 5-22.

Biodiversity Explorer. 2000. Genus: Gladiolus. Available from https://www.biodiversityexplorer.info/plants/iridaceae/gladiolus.htm [accessed 20 Jul. 2020].

BIOPAMA. 2011. Ceres Bergfynbos Reserve. Available from https://rris.biopama.org/pa/300356 [accessed 10 Jul. 2020].

Chen H., Rangasamy M., Tan S.Y., Wang H. \& Siegfried B.D. 2010. Evaluation of five methods for total DNA extraction from western corn rootworm beetles. PLOS ONE 5: e11963.

https://doi.org/10.1371/journal.pone.0011963

Christenhusz M.J.M. \& Byng J.W. 2016. The number of known plants species in the world and its annual increase. Phytotaxa 261 (3): 201-217. https://doi.org/10.11646/phytotaxa.261.3

Critical Ecosystem Partnership Fund. 1996. Eastern Afromontane. Available from https://www.cepf.net/our-work/biodiversity-hotspots/eastern-afromontane [accessed 1 Jul. 2020].

Curran C.H. 1939. Records and descriptions of African Syrphidae (Diptera). III American Museum Novitates 1025: 1-11.

De Menocal P. 2004. African climate change and faunal evolution during the Pliocene-Pleistocene. Earth and Planetary Science Letters 220: 3-24. https://doi.org/10.1016/S0012-821X(04)00003-2

Dirickx H.G. 1998. Catalogue synonymique et géographique des Syrphidae (Diptera) de la Région Afrotropicale. Vol. 2. Muséum d' histoire naturelle, Ville de Genève, Suisse.

Djan M., Ståhls G., Veličković N., Ačanski J., Vidaković D.O., Rojo S., Pérez-Bañón S., Radenković S. \& Vujić A. 2020. The Merodon planifacies subgroup (Diptera: Syrphidae): congruence of molecular and morphometric evidences reveal new taxa in Drakensberg mountains valleys (Republic of South Africa). Zoologischer Anzeiger 287: 105-120. https://doi.org/10.1016/j.jcz.2020.05.010

Doczkal D. \& Pape T. 2009. Lyneborgimyia magnifica gen. et sp. n. (Diptera: Syrphidae) from Tanzania, with a phylogenetic analysis of the Eumerini using new morphological characters. Systematic Entomology 34 (3): 559-573. https://doi.org/10.1111/j.1365-3113.2009.00478.x

Evenhuis N.L. \& Pape T. 2020. Systema Dipterorum, Version 2.6. Available from http://diptera.org/ [accessed 2 Jun. 2020].

Folmer O., Black M., Hoeh W., Lutz R. \& Vrijenhoek R. 1994 DNA primers for amplification of mitochondrial cytochrome c oxidase subunit I from diverse metazoan invertebrates. Molecular Marine Biology and Biotechnology 3: 294-299.

Goldblatt P. \& Manning J. 1998. Gladiolus in Southern Africa. Fernwood Press, Cape Town.

Goldblatt P., Davies J., Manning J., van der Bank M. \& Savolainen V. 2006. Phylogeny of Iridaceae Subfamily Crocoideae based on a combined multigene plastid DNA analysis. Aliso 22 (1): 399-411. https://doi.org/10.5642/aliso.20062201.32

Hadley A. 2006. CombineZ, Ver. 5. Published by the author.

Hijmans R.J., Guarino L. \& Mathur P. 2012. A geographic information system for the analysis of species distribution data. Ver. 7.5. LizardTech, Inc., University of California. 
VUJIĆ A. et al., Revision of the Merodon bombiformis species group

Hall T.A. 1999. BioEdit: a user-friendly biological sequence alignment editor and analysis program for windows 95/98/NT. Nucleic Acids Symposium Series 41: 95-98.

Hull F.M. 1944. Some flies of the Family Syrphidae in the British Museum (Natural History). Annals and Magazine of Natural History Series 1111 (73): 21-66. https://doi.org/10.1080/00222934408527401

Hurkmans W. 1993. A monograph of Merodon (Diptera: Syrphidae). Part 1. Tijdschrift voor Entomologie 136: $147-234$.

International Commission on Zoological Nomenclature. 1999. International Code of Zoological Nomenclature (4th edition) [Incorporating Declaration 44, amendments of Article 74.7.3, with effect from 31 December 1999 and the Amendment on e-publication, amendments to Articles 8, 9, 10, 21 and 78, with effect from 1 January 2012]. International Trust for Zoological Nomenclature, London. Available from https://www.iczn.org/the-code-online/ [accessed 17 Jul. 2020].

Katoh K. \& Standley D.M. 2013. MAFFT multiple sequence alignment software. Ver.7: improvements in performance and usability. Molecular Biology and Evolution 30: 772-780.

https://doi.org/10.1093/molbev/mst010

Kumar S., Stecher G. \& Tamura K. 2016. MEGA7: molecular evolutionary genetics analysis version 7.0 for biggest datasets. Molecular Biology and Evolution 33: 1870-1874.

https://doi.org/10.1093/molbev/msw054

Macquart J. 1855. Diptères exotiques nouveaux ou peu connus. $5^{\mathrm{e}}$ supplément. Memoires de la Société (royale) des Sciences, de l'Agriculture et des Arts à Lille 1 (2): 25-156. Also published separately as his "Diptères exotiques nouveaux ou peu connus. Supplément V": 5-136.

Marcos-García M.Á., Vujić A. \& Mengual X. 2007. Revision of Iberian species of the genus Merodon (Diptera: Syrphidae). European Journal of Entomology 104: 531-572.

https://doi.org/10.14411/eje.2007.073

Martínez-Azorín M., Crespo M.B., Juan A. \& Fay M.F. 2011. Molecular phylogenetics of subfamily Ornithogaloidae (Hyacinthaceae) based on nuclear and plastid DNA regions, including a new taxonomic arrangement. Annals of Botany 107 (1): 1-37. https://doi.org/10.1093/aob/mcq207

McAlpine J.F. 1981. Morphology and Terminology - Adults. In: McAlpine J.F., Peterson B.V., Shewell G.E., Teskey H.J., Vockeroth J.R. \& Wood D.M. (eds) Manual of Nearctic Diptera. Vol. 1: 9-63. Monograph No. 27, Research Branch, Agriculture Canada, Ottawa.

McWilliam H., Li W., Uludag M., Squizzato S., Mi Park Y., Buso N., Cowley A.P. \& Lopez R. 2013. Analysis tool web services from the EMBL-EBI. Nucleic Acids Research 41: W597-W600.

https://doi.org/10.1093/nar/gkt376

Mengual X., Ståhls G., Vujić A. \& Marcos-García M.A. 2006. Integrative taxonomy of Iberian Merodon species (Diptera, Syrphidae). Zootaxa 1377 (1): 1-26. https://doi.org/10.11646/zootaxa.1377.1.1

Miller M.A., Pfeiffer W. \& Schwartz T. 2010. Creating the CIPRES Science Gateway for inference of large phylogenetic trees. 2010 Gateway Computing Environments Workshop (GCE): 1-8. New Orleans, LA. https://doi.org/10.1109/GCE.2010.5676129

Pfosser M.F. \& Speta F. 2004. From Scilla to Charibdis-is our voyage safer now? Plant Systematics and Evolution 246: 245-263.

Preradović J., Andrić A., Radenković S., Šašić Zorić Lj., Pérez-Bañón C., Campoy A. \& Vujić A. 2018. Pupal stages of three species of the phytophagous genus Merodon Meigen (Diptera: Syrphidae). Zootaxa 4420 (2): 229-242. https://doi.org/10.11646/zootaxa.4420.2.5 
Radenković S., Veličković N., Ssymank A., Obreht Vidaković D., Djan M., Ståhls G., Veselić S. \& Vujić A. 2018. Close relatives of Mediterranean endemo-relict hoverflies (Diptera, Syrphidae) in South Africa: Morphological and molecular evidence in the Merodon melanocerus subgroup. PLoS ONE 13 (7): e0200805. https://doi.org/10.1371/journal.pone.0200805

Ricarte A., Marcos-García M.Á. \& Rotheray G.E. 2008. The early stages and life histories of three Eumerus and two Merodon species (Diptera: Syrphidae) from the Mediterranean region. Entomologica Fennica 19: 19-141. https://doi.org/10.33338/ef.84424

Ricarte A., Souba-Dols G.J., Hauser M. \& Marcos-García M.Á. 2017. A review of the early stages and host plants of the genera Eumerus and Merodon (Diptera: Syrphidae), with new data on four species. PLoS ONE 12 (12): e0189852. https://doi.org/10.1371/journal.pone.0189852

Rodríguez F., Oliver J.L., Marín A. \& Medina J.R. 1990. The general stochastic model of nucleotide substitution. Journal of Theoretical Biology 142: 485-501.

https://doi.org/10.1016/S0022-5193(05)80104-3

Rögl F. 1998. Paleogeographic consideration for Mediterranean and Paratethys seaways (Oligocene to Miocene). Annalen des Naturhistorischen Museums in Wien 99A: 279-310.

Šašić Lj., Ačanski J., Vujić A., Ståhls G., Radenković S., Milić D., Obreht-Vidaković D. \& Đan, M. 2016. Molecular and morphological inference of three cryptic species within the Merodon aureus species group (Diptera: Syrphidae). PLoS ONE 11: e0160001. https://doi.org/10.1371/journal.pone.0160001

Sayre R., Comer P. , Hak J., Josse C., Bow J., Warner H., Larwanou M., Kelbessa E., Bekele T., Kehl H., Amena R., Andriamasimanana R., Ba T., Benson L., Boucher T., Brown M., Cress J., Dassering O., Friesen B., Gachathi F., Houcine S., Keita M., Khamala E., Marangu D., Mokua F., Morou B., Mucina L., Mugisha S., Mwavu E., Rutherford M., Sanou P., Syampungani S., Tomor B., Vall A., Vande Weghe J., Wangui E., \& Waruingi L. 2013. A New Map of Standardized Terrestrial Ecosystems of Africa. Washington, DC: Association of American Geographers. Available from http://www.aag.org/cs/publications/special/map_african_ecosystems [accessed 24 May 2021].

Smith K.G.V. \& Vockeroth J.R. 1980. Family Syrphidae. In: Crosskey R.W. (ed.) Catalogue of the Diptera of the Afrotropical Region: 488-510. British Museum, London.

South African National Biodiversity Institute. 2004. Leucosidea sericea. Available from http://pza.sanbi.org/leucosidea-sericea [accessed 21 Jun. 2020].

Speight M.C.D. 2020. Species accounts of European Syrphidae, 2020. Syrph the Net, the database of European Syrphidae (Diptera) vol. 104. Syrph the Net publications, Dublin.

Ståhls G., Vujić A., Pérez-Bañón C., Radenković S., Rojo S. \& Petanidou T. 2009. COI barcodes for identification of Merodon hoverflies (Diptera, Syrphidae) of Lesvos Island, Greece. Molecular Ecology Resources 9 (6): 1431-1438. https://doi.org/10.1111/j.1755-0998. 2009.02592.x

Stamatakis A. 2014. RAxML version 8: a tool for phylogenetic analysis and post-analysis of large phylogenies. Bioinformatics 30: 1312-1313. https://doi.org/10.1093/bioinformatics/btu033

Stuckenberg B.R. 1956. The immature stages of Merodon bombiformis Hull, a potential pest of bulbs in South Africa. (Diptera: Syrphidae). Journal of the Entomological Society of Southern Africa 19 (2): 219-224.

Thompson F.C. 1988. Syrphidae (Diptera) described from unknown localities. Journal of the New York Entomological Society 96 (2): 200-226.

Thompson F.C. 1999. A key to the genera of the flower flies (Diptera: Syrphidae) of the Neotropical Region including descriptions of new genera and species and a glossary of taxonomic terms. Contributions on Entomology, International 3: 321-378. 
Vujić A., Radenković S., Ståhls G., Ačanski J., Stefanović A., Veselić S., Andrić A. \& Hayat R. 2012. Systematics and taxonomy of the ruficornis group of genus Merodon Meigen (Diptera: Syrphidae). Systematic Entomology 37: 578-602. https://doi.org/10.1111/j.1365-3113. 2012.00631.x

Vujić A., Radenković S. \& Likov L. 2018. Revision of the Palaearctic species of the Merodon desuturinus group (Diptera, Syrphidae). ZooKeys 771: 105-138. https://doi.org/10.3897/zookeys.771.20481

Vujić A., Radenković S., Likov L., Andrić A., Gilasian E. \& Barkalov A. 2019a. Two new enigmatic species of the genus Merodon Meigen (Diptera: Syrphidae) from the north-eastern Middle East. Zootaxa 4555 (2): 187-208. htps://doi.org/10.11646/zootaxa.4555.2.2

Vujić A., Ståhls G. \& Radenković S. 2019b. Hidden European diversity: a new monotypic hoverfly genus (Diptera: Syrphidae: Eristalinae: Rhingiini). Zoological Journal of the Linnean Society 185 (4): 1188-1211. https://doi.org/10.1093/zoolinnean/zly066

Vujić A., Speight M., de Courcy Williams M., Rojo S., Ståhls G., Radenković S., Likov L., Miličić M., Pérez-Bañón C., Falk S. \& Petanidou T. 2020. Atlas of the Hoverflies of Greece. Brill, Leiden, The Netherlands. https://doi.org/10.1163/9789004334670

Manuscript received: 6 November 2020

Manuscript accepted: 12 April 2021

Published on: 21 June 2021

Topic editor: Nesrine Akkari

Section editor: Torbjørn Ekrem

Desk editor: Pepe Fernández

Printed versions of all papers are also deposited in the libraries of the institutes that are members of the EJT consortium: Muséum national d'histoire naturelle, Paris, France; Meise Botanic Garden, Belgium; Royal Museum for Central Africa, Tervuren, Belgium; Royal Belgian Institute of Natural Sciences, Brussels, Belgium; Natural History Museum of Denmark, Copenhagen, Denmark; Naturalis Biodiversity Center, Leiden, the Netherlands; Museo Nacional de Ciencias Naturales-CSIC, Madrid, Spain; Real Jardín Botánico de Madrid CSIC, Spain; Zoological Research Museum Alexander Koenig, Bonn, Germany; National Museum, Prague, Czech Republic.

\section{Supplementary material}

Supp. file 1: Data related to the studied specimens and the corresponding GenBank accession numbers. https://doi.org/10.5852/ejt.2021.755.1401.4469 